\title{
EXTENSION OF TWO-DIMENSIONAL MEAN CURVATURE FLOW WITH FREE BOUNDARY
}

\author{
SIAO-HAO GUO
}

\begin{abstract}
Given a mean curvature flow of compact, embedded $C^{2}$ surfaces satisfying Neumann free boundary condition on a mean convex, smooth support surface in 3-dimensional Euclidean space, we show that it can be extended as long as its mean curvature and perimeter stay uniformly bounded along the flow.
\end{abstract}

\section{INTRODUCTION}

Mean curvature flow (MCF) of hypersurfaces in Euclidean space is a one-parameter family of hypersurfaces $\left\{\Sigma_{t}\right\}_{a<t<b}$ whose normal velocity equals the mean curvature vector. Namely,

$$
\left(\partial_{t} X_{t}\right)^{\perp}=\vec{H}_{\Sigma_{t}},
$$

where $X_{t}$ is the position vector of $\Sigma_{t}$ and $\vec{H}_{\Sigma_{t}}=\triangle_{\Sigma_{t}} X_{t}$ is the mean curvature vector. Given a closed, embedded, $C^{2}$ hypersurface $\Sigma_{0}$, there is a unique MCF $\left\{\Sigma_{t}\right\}$ starting from it. After a finite time, say as $t \nearrow T$, the flow will become singular. One can see this by comparing the flow with the evolution of any enclosing sphere using the maximum principle. Moreover, singularities of the flow always accompany the blow up of the second fundamental form (cf. [E]), i.e.

$$
\limsup _{t \nearrow T}\left\|A_{\Sigma_{t}}\right\|_{L^{\infty}}=\infty
$$

where $A_{\Sigma_{t}}=\nabla_{\Sigma_{t}}^{2} X_{t} \cdot N_{\Sigma_{t}}$ is the second fundamental form of $\Sigma_{t}$ and $N_{\Sigma_{t}}$ is the unit normal vector. A natural question is whether the mean curvature must also blow up at the first singular time. If so, singularities can be interpreted as the blow up of the normal speed.

Under various assumptions, the mean curvature does blow up at the first singular time. For instance, in [HS] it is shown that if the initial hypersurface is closed and mean convex (i.e. the mean curvature vector points to the inward direction), the second fundamental form will be bounded by a multiple of the mean curvature, which forces the mean curvature to blow up at the first singular time. More examples can be seen in [C, LS, LW, LZZ. In particular, Li-Wang in [LW] show that the $\mathrm{MCF}$ of closed surfaces in $\mathbb{R}^{3}$ can be extended so long as the mean curvature stays uniformly bounded. This tells us that singularities of MCF of closed surfaces in $\mathbb{R}^{3}$ can be characterized by the blow-up of the mean curvature.

On the other hand, people also consider MCF subject to various boundary conditions, in particular the "free boundary condition". Given an open, connected domain $U$ in Euclidean space with smooth boundary, a flow $\left\{\Sigma_{t}\right\}$ living in $U$ satisfies the "free boundary condition" provided that it meets $\Gamma=\partial U$ orthogonally at 
every time. Namely,

$$
N_{\Sigma_{t}} \cdot \nu=0 \quad \text { on } \quad \partial \Sigma_{t}=\Sigma_{t} \cap \Gamma,
$$

where $\nu$ is the inward, unit normal vector of $\Gamma$. As in the boundaryless case, given a compact, embedded, $C^{2}$ hypersurface $\Sigma_{0}$ meeting $\Gamma$ orthogonally, there is a unique $\operatorname{MCF}\left\{\Sigma_{t}\right\}$ which starts from it and has free boundary on $\Gamma$ (cf. [S] $)$. The flow may have long-time existence under certain conditions (for instance, see [H1]). However, if the flow becomes singular in finite time, the second fundamental form must blow up (cf. $[\mathrm{S}])$.

In this paper, we investigate the extension problem for MCF of surfaces with free boundary so as to gain insight into the development of singularities. The support surface $\Gamma=\partial U$ is assumed to be smooth and mean convex (i.e. the mean curvature vector of $\Gamma$ points toward $U$ ), which is used to ensure the "boundary approaches boundary" condition (see $[\mathrm{K}]$ for more details). In Theorem 6.1 we show that any $\operatorname{MCF}\left\{\Sigma_{t}\right\}_{0<t<T}$ moving freely in $U$ (i.e. satisfying the free boundary condition) can be extended as long as its mean curvature and perimeter stay uniformly bounded, i.e.

$$
\sup _{0 \leq t<T}\left(\left\|H_{\Sigma_{t}}\right\|_{L^{\infty}}+\mathcal{H}^{1}\left(\partial \Sigma_{t}\right)\right)<\infty,
$$

where $H_{\Sigma_{t}}=\vec{H}_{\Sigma_{t}} \cdot N_{\Sigma_{t}}$ is the mean curvature of $\Sigma_{t}, \mathcal{H}^{k}$ denotes $k$-dimensional Hausdorff measure, and $T>0$ is a finite number. This can be thought of as a generalization of [LW], in which surfaces are assume to be closed. In fact, our proof parallels that of Li-Wang in [LW]. We adapt their argument to the free boundary setting by using the method of reflection and many crucial properties for MCF with free boundary (cf. [B, GJ, $\mathrm{K}, \mathrm{S}$ ). The reason for assuming the perimeter bound (compared with the assumption in [LW]) comes from the use of the Gauss-Bonnet theorem (see Section 4). A key part of the proof is to show that every limit point $P$ of the flow $\left\{\Sigma_{t}\right\}_{0 \leq t<T}$ as $t \nearrow T$ satisfies

$$
\Theta_{\left\{\Sigma_{t}\right\}}(P, T)=\left\{\begin{array}{ll}
1, & \text { if } P \in U \\
\frac{1}{2}, & \text { if } P \in \Gamma
\end{array},\right.
$$

where $\Theta_{\left\{\Sigma_{t}\right\}}$ is Huisken's density (see [E, $[\mathrm{K}$ or Lemma 2.7 for the definition). However, unlike [LW], where one can apply White's regularity theorem (cf. [Wh]) to conclude that the flow is smooth up to time $T$, here we need an additional argument to handle possible boundary singularities (see Section 6).

The organization of the paper is as follows. In Section 2 fundamental tools for dealing with surfaces with free boundary are introduced. These include the parametrization of the tubular neighborhood of a surface and the reflection principle for parabolic equations with homogeneous Neumann boundary condition. In addition, the crucial monotonicity formulas for $\mathrm{MCF}$ (cf. [H2, E, B]) and the compactness theorem in the free boundary setting are also included. In Section 3 , key estimates for MCF with free boundary are derived. More precisely, Li-Wang's pseudolocality theorem and small energy theorem are adapted to the free boundary setting. Then Section 4-Section 6 are devoted to the proof of the main theorem. Specifically, in Section 4 we formulate the assumptions and prove the condensation compactness theorem for sequences of parabolic rescaling of the flow. In Section [5. we follow [LW to prove (1.1). Finally, in Section 6 we complete the proof. The arguments in Section 3 - Section 5 parallel that of [LW]. 


\section{ACKNOWLEDGEMENT}

The author is grateful to Peter Sternberg for suggesting the problem, for insightful discussions and for providing helpful comments on this paper.

\section{Preliminaries}

In this section we will set up the tools which are required for studying MCF with free boundary. These can be roughly divided into four different topics as follows.

(1) Complementary balls, the $\kappa$-graph condition of surfaces, and the parametrization of a tubular neighborhood.

(2) Huisken's monotonicity formula for MCF and Buckland's adaption to MCF with free boundary.

(3) Stahl's gradient estimates for MCF with free boundary, parametrization of MCF near the free boundary, the reflection principle for parabolic equations with Neumann boundary condition, and the $C^{2,1}$ estimates for MCF with free boundary.

(4) Topology and compactness of the space of MCF with free boundary.

Note that the discussion of the above will be in the form of surfaces in $\mathbb{R}^{3}$ for definiteness; however, most of them work in higher dimensions as well.

Let's begin with the first topic. Given an embedded, smooth surface $\Gamma$ in $\mathbb{R}^{3}$, let $X$ be a point which lies in a tubular neighborhood of $\Gamma$. Recall that the projection of $X$ on $\Gamma$ is given by

$$
\stackrel{\circ}{X}:=X-d_{\Gamma}(X) \nabla d_{\Gamma}(X),
$$

where $d_{\Gamma}(X)=\operatorname{dist}(X, \Gamma)$, and the reflection of $X$ with respect to $\Gamma$ is given by

$$
\tilde{X}:=X-2 d_{\Gamma}(X) \nabla d_{\Gamma}(X)=2 \stackrel{\circ}{X}-X .
$$

Below we follow GJ] to define the complementary balls.

Definition 2.1. (Complementary Balls)

Let $U$ be an open, connected subset of $\mathbb{R}^{3}$ with an embedded, smooth boundary surface $\Gamma$. Given $r>0$ and a point $P \in U$ which lies in the tubular neighborhood of $\Gamma$, define

$$
\tilde{B}_{r}(P)=\left\{X \in U \mid \tilde{X} \in B_{r}(P) \backslash U\right\}
$$

where $B_{r}(P)$ is the open ball in $\mathbb{R}^{3}$ centered at $P$ with radius $r$. Note that $\tilde{B}_{r}(P)=$ $\emptyset$ if $B_{r}(P) \subset U$.

In other words, $\tilde{B}_{r}(P)$ is the reflection of $B_{r}(P) \backslash U$ with respect to $\Gamma$. The prototype of reflection is with respect to a plane. What follows is a simple remark on the reflection and complementary balls with respect to a plane.

Remark 2.2. If

$$
U=\mathbb{R}_{+}^{3}=\left\{\left(x_{1}, x_{2}, x_{3}\right) \mid x_{2}>0\right\}
$$

is a half-space, then

$$
\Gamma=\partial U=\left\{\left(x_{1}, x_{2}, x_{3}\right) \mid x_{2}=0\right\}=x_{1} x_{3}-\text { plane }
$$

and the reflection with respect to $\Gamma$ is given by

$$
\left(\widetilde{x_{1}, x_{2}, x_{3}}\right)=\left(x_{1},-x_{2}, x_{3}\right) \text {. }
$$


Let $\Sigma$ be a surface in $U$ which meets $\Gamma$ orthogonally. Define $\tilde{\Sigma}$ to be the reflection of $\Sigma$ with respect to $\Gamma$. Namely,

$$
\tilde{\Sigma}=\{X \mid \tilde{X} \in \Sigma\} .
$$

Given $P \in \Sigma$ and $r>0$, note that $\Sigma \cap \tilde{B}_{r}(P)$ is the reflection of $\tilde{\Sigma} \cap B_{r}(P)$ with respect to $\Gamma$, and hence

$$
\mathcal{H}^{2}\left(\Sigma \cap \tilde{B}_{r}(P)\right)=\mathcal{H}^{2}\left(\tilde{\Sigma} \cap B_{r}(P)\right) .
$$

Let $\bar{\Sigma}=\Sigma \cup \tilde{\Sigma}$ be the extension of $\Sigma$ across $\Gamma$, then we have

$$
\begin{gathered}
\mathcal{H}^{2}\left(\bar{\Sigma} \cap B_{r}(P)\right)=\mathcal{H}^{2}\left(\Sigma \cap B_{r}(P)\right)+\mathcal{H}^{2}\left(\tilde{\Sigma} \cap B_{r}(P)\right) \\
=\mathcal{H}^{2}\left(\Sigma \cap B_{r}(P)\right)+\mathcal{H}^{2}\left(\Sigma \cap \tilde{B}_{r}(P)\right) .
\end{gathered}
$$

In addition, suppose that $\Sigma$ is a $\delta$-Lipschitz graph for some $\delta>0$, say

$$
\Sigma=\left\{\left(x_{1}, x_{2}, u\left(x_{1}, x_{2}\right)\right) \mid x_{1} \in \mathbb{R}, x_{2} \geq 0\right\},
$$

where the function satisfies $\partial_{2} u\left(x_{1}, 0\right)=0$ (since $\Sigma$ meets $\Gamma$ orthogonally) and $|\nabla u| \leq \delta$ (due to the $\delta$-Lipschitz graph condition). Then there holds

$$
\frac{\mathcal{H}^{2}\left(\Sigma \cap B_{r}(P)\right)+\mathcal{H}^{2}\left(\Sigma \cap \tilde{B}_{r}(P)\right)}{\pi r^{2}} \leq \sqrt{1+\delta^{2}} .
$$

Note that the above area ratio estimate also holds for non-flat $\Gamma$, as long as $r$ is sufficiently small (depending on the second fundamental form of $\Gamma$ and $\Sigma$, see Lemma 3.11).

We then proceed to introduce the $\kappa$-graph condition. Throughout the paper, we use $\nu$ to denote the unit normal vector of $\Gamma$. Moreover, if $\Gamma=\partial U$, where $U$ is an open, connected subset of $\mathbb{R}^{3}$, then $\nu$ is assumed to be the inward normal vector (i.e. pointing toward $U$ ).

Definition 2.3. $(\kappa-$ Graph Condition)

A properly embedded $C^{3,1}$ surface $\Gamma$ in $\mathbb{R}^{3}$ satisfies the " $\kappa$-graph condition" for some $\kappa>0$ if the following property holds. Choose an arbitrary point on $\Gamma$; without loss of generality, we may assume that the chosen point is $O$ and $\nu(O)=(0,1,0)$. Then $\Gamma \cap B_{\kappa^{-1}}(O)$ is a graph of a $C^{3,1}$ function $\varphi$ defined in $T_{O} \Gamma=x_{1} x_{3}$-plane, i.e. $x_{2}=\varphi\left(x_{1}, x_{3}\right)$ and

$$
\varphi(0,0)=0, \quad \nabla \varphi(0,0)=(0,0) .
$$

Moreover, the function $\varphi$ satisfies

$$
\left|\nabla^{2} \varphi\right| \leq \kappa, \quad\left|\nabla^{3} \varphi\right| \leq \kappa^{2}, \quad\left[\nabla^{3} \varphi\right]_{1} \leq \kappa^{3},
$$

where $[\cdot]_{1}$ is the Lipschitz norm defined by

$$
[v]_{1}=\sup _{x \neq x^{\prime}} \frac{\left|v(x)-v\left(x^{\prime}\right)\right|}{\left|x-x^{\prime}\right|} .
$$

Note that the mean value theorem then yields

$$
\left|\frac{\varphi(\xi)}{\xi}\right|+|\nabla \varphi(\xi)| \lesssim \kappa|\xi|
$$

for $|\xi| \lesssim \kappa^{-1}$ 
Any embedded, smooth surface $\Gamma$ would locally satisfy the above condition. More precisely, given $R>0$, there exists $\kappa>0$ so that $\Gamma \cap B_{R}(O)$ satisfies the $\kappa$-graph condition. Consequently, if the involved domain is bounded, then for simplicity we may just assume that $\Gamma$ satisfies the $\kappa$-graph condition for some $\kappa>0$.

In follows we discuss the parametrization of a tubular neighborhood of $\Gamma$. This will be used repeatedly to study the regularity of surfaces near the boundary.

Definition 2.4. (Parametrization of Tubular Neighborhood)

Let $U$ be an open, connected subset of $\mathbb{R}^{3}$ whose boundary $\Gamma=\partial U$ satisfies the $\kappa$-graph condition for some $\kappa>0$. Choose an arbitrary point on $\Gamma$; without loss of generality, we may assume that the chosen point is $O, \nu(O)=(0,1,0)$, and that $\Gamma \cap B_{\kappa^{-1}}(O)$ is defined by $x_{2}=\varphi\left(x_{1}, x_{3}\right)$ as in Definition [2.3. Then we can parametrize $B_{\kappa^{-1}}(O)$ by

$$
\left(x_{1}, x_{2}, x_{3}\right)=\Phi\left(y_{1}, y_{2}, y_{3}\right):=\left(y_{1}, \varphi\left(y_{1}, y_{3}\right), y_{3}\right)+y_{2} \nu
$$

where

$$
\nu=\nu\left(y_{1}, y_{3}\right)=\frac{\left(-\partial_{1} \varphi, 1,-\partial_{3} \varphi\right)}{\sqrt{1+|\nabla \varphi|^{2}}}
$$

is the inward, unit normal vector of $\Gamma \cap B_{\kappa^{-1}}(O)$. Note that $\Phi$ is a $C^{2,1}$ diffeomorphism from a neighborhood of $O$ onto $B_{\kappa^{-1}}(O)$ and that

$$
U \cap B_{\kappa^{-1}}(O)=\Phi\left\{y_{2}>0\right\}, \quad \Gamma \cap B_{\kappa^{-1}}(O)=\Phi\left\{y_{2}=0\right\} .
$$

Moreover, we have

$$
\stackrel{\circ}{X}=\stackrel{\circ}{X}\left(y_{1}, y_{3}\right)=\left(y_{1}, \varphi\left(y_{1}, y_{3}\right), y_{3}\right), \quad d_{\Gamma}(X)=y_{2}, \quad \nabla d_{\Gamma}(X)=\nu(\stackrel{\circ}{X}),
$$

where $\stackrel{\circ}{X}$ is the projection of $X$ on $\Gamma$ and $d_{\Gamma}(X)=\operatorname{dist}(X, \Gamma)$.

Following the notations in Definition 2.4, we have

$$
\begin{gathered}
\Phi(Y)-Y=\left(0, \varphi\left(y_{1}, y_{3}\right), 0\right)+y_{2}\{\nu-(0,1,0)\} \\
\partial_{1} \Phi=\left(1, \partial_{1} \varphi, 0\right)+y_{2} \partial_{1} \nu, \quad \partial_{2} \Phi=\nu(\stackrel{\circ}{X}), \quad \partial_{3} \Phi=\left(0, \partial_{3} \varphi, 1\right)+y_{2} \partial_{3} \nu
\end{gathered}
$$

which implies, by Definition 2.3, that $\Phi(O)=O, D \Phi(O)=I$ (the identity $3 \times 3$ matrix), and

$$
\begin{gathered}
|\Phi(Y)-Y| \lesssim \kappa|Y|^{2}, \quad|D \Phi(Y)-I| \lesssim \kappa|Y| \\
\left|D^{2} \Phi(Y)\right| \lesssim \kappa, \quad\left[D^{2} \Phi(Y)\right]_{1} \lesssim \kappa^{2} .
\end{gathered}
$$

Notice that $\Phi$ not only maps $\left\{y_{2}=0\right\}$ onto $\Gamma \cap B_{\kappa^{-1}}(O)$ but also preserves their unit normal vectors and the reflection with respect to them, i.e.

$$
\begin{gathered}
(0,1,0) \stackrel{D \Phi}{\mapsto} \nu, \\
\Phi(\tilde{Y})=\widetilde{\Phi(Y)} \Leftrightarrow \Phi^{-1}(\tilde{X})=\widetilde{\Phi^{-1}(X)},
\end{gathered}
$$

where $\tilde{Y}$ is the reflection of $Y$ with respect to the plane $\left\{y_{2}=0\right\}$ (see Remark 2.2) and $\widetilde{\Phi(Y)}$ is the reflection of $\Phi(Y)$ with respect to $\Gamma ; \tilde{X}$ and $\widetilde{\Phi^{-1}(X)}$ are defined anaglously. Furthermore, let

$$
h_{i j}(Y)=\partial_{i} \Phi(Y) \cdot \partial_{j} \Phi(Y), \quad i, j \in\{1,2,3\}
$$


be the pull-back metric, and

$$
\Gamma_{i j}^{k}(Y)=\frac{1}{2} h^{k l}(Y)\left\{\partial_{i} h_{j l}(Y)+\partial_{j} h_{i l}(Y)-\partial_{l} h_{i j}(Y)\right\}
$$

be the Levi-Civita connection, where $h^{k l}(Y)$ is the inverse of $h_{k l}(Y)$. Then we have

$$
\begin{gathered}
\left|h_{i j}-\boldsymbol{\delta}_{i j}\right| \lesssim \kappa|Y|, \quad\left|\Gamma_{i j}^{k}\right| \lesssim \kappa, \quad\left[\Gamma_{i j}^{k}\right]_{1} \lesssim \kappa^{2}, \\
h_{22}\left(y_{1}, y_{2}, y_{3}\right)=1, \quad h_{12}\left(y_{1}, 0, y_{3}\right)=0=h_{32}\left(y_{1}, 0, y_{3}\right),
\end{gathered}
$$

where $\boldsymbol{\delta}_{i j}$ is Kronecker delta.

We conclude the first topic with the following remark on the scaling property of the parametrization in Definition 2.4.

Remark 2.5. Following the notations of Definition 2.4 for each $\lambda>0$ we define $\Gamma^{\lambda}=\frac{1}{\lambda} \Gamma$. Apparently $\Gamma^{\lambda}$ satisfies the $\lambda \kappa$-graph condition and $\Gamma^{\lambda} \cap B_{(\lambda \kappa)^{-1}}(O)$ is a graph of

$$
\varphi^{\lambda}\left(y_{1}, y_{3}\right):=\frac{1}{\lambda} \varphi\left(\lambda y_{1}, \lambda y_{3}\right) .
$$

Let $\Phi^{\lambda}$ be the map corresponding to to $\Gamma^{\lambda}$ (as in Definition 2.4), then it's not hard to see that

$$
X=\Phi(Y) \Leftrightarrow \frac{1}{\lambda} X=\Phi^{\lambda}\left(\frac{1}{\lambda} Y\right) .
$$

In other words, the pull-back of $B_{(\lambda \kappa)^{-1}}(O)$ by $\Phi^{\lambda}$ corresponds to the pull-back of $B_{\kappa^{-1}}(O)$ by $\Phi$ via scaling.

Next, we begin the second topic with the fundamental monotonicity formulas for MCF. The formula was first discovered by Huisken (cf. [H2 ) for complete MCF, then Ecker found the localized version (cf. [E]). Afterward, Buckland modified the formula to work for MCF with free boundary (cf. [B]).

Note that for a given a surface $\Sigma$, we denote its second fundamental form by $A_{\Sigma}$, mean curvature by $H_{\Sigma}$ and unit normal vector by $N_{\Sigma}$.

Lemma 2.6. (Monotonicity Formulas for $M C F$ )

Let $\left\{\Sigma_{t}\right\}_{0<t<T}$ be a properly embedded $C^{2} M C F$ moving freely in $U \subset \mathbb{R}^{3}$, where $T>0$ is a finite number. Assume that $\Gamma=\partial U$ satisfies the $\kappa$-graph condition for some $\kappa>0$. Then we have the following.

- Given $P \in U$ and $0<r<\frac{1}{2 \sqrt{5}} d_{\Gamma}(P)$, there holds

$$
\begin{gathered}
\frac{d}{d t} \int_{\Sigma_{t}} \psi_{r ; P, T} \Psi_{P, T}(X, t) d \mathcal{H}^{2}(X) \\
\leq-\int_{\Sigma_{t}}\left(H_{\Sigma_{t}}-\nabla \ln \Psi_{P, T} \cdot N_{\Sigma_{t}}\right)^{2} \psi_{r ; P, T} \Psi_{P, T}(X, t) d \mathcal{H}^{2}(X)
\end{gathered}
$$

for $T-r^{2} \leq t<T$, where

$$
\psi_{r ; P, T}(X, t)=\left(1-\frac{|X-P|^{2}-4(T-t)}{r^{2}}\right)_{+}^{3}
$$

is a localization function and

$$
\Psi_{P, T}(X, t)=\frac{1}{4 \pi(T-t)} \exp \left(-\frac{|X-P|^{2}}{4(T-t)}\right)
$$


is the backward heat kernel centered at $(P, T)$. Note that on the right side of the formula, the first factor in the integrand can be written explicitly as

$$
H_{\Sigma_{t}}-\nabla \ln \Psi_{P, T} \cdot N_{\Sigma_{t}}=H_{\Sigma_{t}}+\frac{(X-P) \cdot N_{\Sigma_{t}}}{2(T-t)} .
$$

- Given $P \in \Gamma$, there holds

$$
\begin{gathered}
\frac{d}{d t} \int_{\Sigma_{t}} e^{85\left(\kappa^{2}(T-t)\right)^{\frac{2}{5}}} \eta_{\Gamma ; P, T} \Psi_{\Gamma ; P, T}(X, t) d \mathcal{H}^{2}(X) \\
\leq-\int_{\Sigma_{t}}\left(H_{\Sigma_{t}}-\nabla \ln \Psi_{\Gamma ; P, T} \cdot N_{\Sigma_{t}}\right)^{2} e^{85\left(\kappa^{2}(T-t)\right)^{\frac{2}{5}}} \eta_{\Gamma ; P, T} \Psi_{\Gamma ; P, T}(X, t) d \mathcal{H}^{2}(X) \\
\text { for } \max \left\{T-\frac{1}{2}\left(\frac{3}{320}\right)^{5} \kappa^{-2}, 0\right\} \leq t<T, \text { where } \\
\eta_{\Gamma ; P, T}(X, t)=\left(1-\frac{|X-P|^{2}+|\tilde{X}-P|^{2}-80(T-t)}{\left(\frac{1}{2}\left(\kappa^{2}(T-t)\right)^{\frac{2}{5}} \kappa^{-1}\right)^{2}}\right)_{+}^{4}
\end{gathered}
$$

is a localization function and

$$
\Psi_{\Gamma ; P, T}(X, t)=\frac{1}{4 \pi(T-t)} \exp \left(-\frac{\frac{1}{2}\left(|X-P|^{2}+|\tilde{X}-P|^{2}\right)}{4\left(1+16\left(\kappa^{2}(T-t)\right)^{\frac{2}{5}}\right)(T-t)}\right)
$$

is the modified backward heat kernel centered at $(P, T)$. Note that $\tilde{X}$ is the reflection of $X$ with respect to $\Gamma$ and that on the right side of the formula, the first factor in the integrand can be written explicitly as

$$
\begin{gathered}
H_{\Sigma_{t}}-\nabla \ln \Psi_{\Gamma ; P, T} \cdot N_{\Sigma_{t}} \\
=H_{\Sigma_{t}}+\frac{(X-P) \cdot N_{\Sigma_{t}}-\left((X-P) \cdot \nabla d_{\Gamma}-d_{\Gamma}\right) \nabla d_{\Gamma} \cdot N_{\Sigma_{t}}-d_{\Gamma} \nabla^{2} d_{\Gamma}\left(X-P, N_{\Sigma_{t}}\right)}{2\left(1+16\left(\kappa^{2}(T-t)\right)^{\frac{2}{5}}\right)(T-t)} .
\end{gathered}
$$

As a corollary, the integrals

$$
\int_{\Sigma_{t}} \psi_{r ; P, T} \Psi_{P, T} d \mathcal{H}^{2} \quad \text { for } P \in U
$$

and

$$
\int_{\Sigma_{t}} e^{85\left(\kappa^{2}(T-t)\right)^{\frac{2}{5}}} \eta_{\Gamma ; P, T} \Psi_{\Gamma ; P, T} d \mathcal{H}^{2} \quad \text { for } P \in \Gamma
$$

are non-negative and non-increasing in $t$, so they must converge as $t \nearrow T$. The limit is called Huisken's density of the flow $\left\{\Sigma_{t}\right\}$ at $(P, T)$, and it can be shown to be independent of the choice $r$ (cf. [E]). We finish the second topic by listing some important properties of Huisken's density as follows (cf. [E, $\mathrm{K}]$ ).

Lemma 2.7. (Huisken's Density)

Following the notations in Lemma 2.6, let

$$
\Theta_{\left\{\Sigma_{t}\right\}}(P, T)=\left\{\begin{array}{c}
\lim _{t \nearrow T} \int_{\Sigma_{t}} \psi_{r ; P, T} \Psi_{P, T} d \mathcal{H}^{2}, \quad \text { if } P \in U \\
\lim _{t \nearrow T} \int_{\Sigma_{t}} e^{85\left(\kappa^{2}(T-t)\right)^{\frac{2}{5}}} \eta_{\Gamma ; P, T} \Psi_{\Gamma ; P, T} d \mathcal{H}^{2}, \quad \text { if } P \in \Gamma
\end{array} .\right.
$$

If the flow $\left\{\Sigma_{t}\right\}_{0 \leq t<T}$ is regular up to time $T$, let $\Sigma_{T}=\lim _{t \nearrow T} \Sigma_{t}$. Then we have 


$$
\Theta_{\left\{\Sigma_{t}\right\}}(P, T)=\left\{\begin{array}{c}
1, \quad \text { if } P \in \Sigma_{T} \cap U \\
\frac{1}{2}, \quad \text { if } P \in \Sigma_{T} \cap \Gamma . \\
0, \quad \text { if } P \notin \Sigma_{T}
\end{array}\right.
$$

Moreover, $\Theta_{\left\{\Sigma_{t}\right\}}(P, T)$ is upper semi-continuous. Namely, given a sequence $t_{i} \nearrow T$ and points $P_{i} \in \Sigma_{t_{i}}$ so that

- $P_{i} \rightarrow P$ as $i \rightarrow \infty$,

- If $P \in \Gamma$, then $P_{i} \in \Gamma$ for $i \gg 1$,

then we have

$$
\Theta_{\left\{\Sigma_{t}\right\}}(P, T) \geq \limsup _{i \rightarrow \infty} \Theta_{\left\{\Sigma_{t}\right\}}\left(P_{i}, t_{i}\right) .
$$

The main point of the third topic is about the $C^{2,1}$ estimates for MCF with free boundary. Since the interior estimate can be found in [E], our focus will be on the boundary regularity. To achieve that, we will first use the map in Definition 2.4 to pull back the flow to a half-space, where the pull-back flow has free boundary on the boundary plane. Then we locally write the flow as a graph of a time-dependent function, which satisfies a parabolic equation and homogeneous Neumann boundary condition. Using the method of reflection, we can extend the function across the boundary and derive the boundary estimate.

Throughout the paper, the following notation will be adopted. Given a surface $\Sigma$ and a point $P \in \Sigma$, we denote by $(\Sigma)_{P}$ the path-connected component of $\Sigma$ containing $P$, i.e.

$$
(\Sigma)_{P}=\{Q \in \Sigma \mid \exists \text { continuous path } \gamma:[0,1] \rightarrow \Sigma \text { s.t. } \gamma(0)=P, \gamma(1)=Q\} .
$$

The following gradient estimate for MCF with free boundary is due to Stahl, who derived the estimate by using the maximum principle (cf. [EH,, $\mathrm{S}]$ ).

Lemma 2.8. (Stahl's Gradient Estimate for MCF)

Given $\varepsilon>0$, there exists $\delta>0$ with the following property. Let $\left\{\Sigma_{t}\right\}_{a \leq t \leq b}$ be a properly embedded $C^{2} M C F$ moving freely in $U \subset \mathbb{R}^{3}$, where $a \leq 0 \leq \bar{b}$ are constants. Assume that $O \in \Sigma_{0}$ and that

- Either $B_{1}(O) \subset U$, or $O \in \Gamma$ and $\Gamma=\partial U$ satisfies the $\kappa$-graph condition for some $0<\kappa \leq 1$;

- There holds

$$
\sup _{a \leq t \leq b}\left\|A_{\Sigma_{t}}\right\|_{L^{\infty}\left(B_{1}(O)\right)} \leq K
$$

for some $K \geq 1$.

Then we have

$$
N_{\Sigma_{t}}(P) \cdot N_{\Sigma_{0}}(O) \geq 1-\varepsilon
$$

for all $P \in\left(\Sigma_{t} \cap B_{\frac{\delta}{K}}(O)\right)_{O_{t}}, \breve{a} \leq t \leq \breve{b}$, where

$$
\breve{a}=\max \left\{a,-\frac{\delta}{K^{2}}\right\}, \quad \breve{b}=\min \left\{b, \frac{\delta}{K^{2}}\right\},
$$

and $O_{t}$ is the normal trajectory of $O$ along the flow, i.e.

$$
O_{t} \in \Sigma_{t} \quad \text { and } \quad \partial_{t} O_{t} \perp T_{O_{t}} \Sigma_{t} .
$$


As a consequence of the above lemma, near the point $O$ and around the time 0 , the flow $\left\{\Sigma_{t}\right\}$ is the graph of a time-dependent function over $T_{O} \Sigma_{0}$ with small gradient. However, if $O \in \Gamma$ and $U$ is not a cylinder, the domain of the function might be time-dependent. To solve this issue, we will use the map in Definition 2.4 to parametrize the tubular neighborhood of $\Gamma$ so as to make the pull-back of the domain (of the defining function) independent of time. Below we will carry out the details of realizing this idea.

Note that Einstein summation convention will be adopted throughout the paper.

Lemma 2.9. (Parametrization of $M C F$ Near the Boundary)

Fix $\iota \in\{0,1\}$. Given $\varepsilon>0$, there exists $\delta>0$ with the following property. Let $\left\{\Sigma_{t}\right\}_{-1<t<\iota}$ be a properly embedded $C^{2} M C F$ moving freely in $U \subset \mathbb{R}^{3}$. Assume that $O \in \bar{\Gamma}=\partial U, \nu(O)=(0,1,0)$ and that

- $\Gamma$ satisfies the $\kappa$-graph condition for some $0<\kappa \leq 1$;

- The second fundamental form of $\left\{\Sigma_{t}\right\}$ satisfies

$$
\sup _{-1 \leq t \leq \iota}\left\|A_{\Sigma_{t}}\right\|_{L^{\infty}\left(B_{1}(O)\right)} \leq K
$$

for some $K \geq 1$;

- There exists $P \in \partial \Sigma_{0} \cap B_{\frac{\delta}{K}}(O)$ so that $N_{\Sigma_{0}}(P) \cdot(0,0,1) \geq 1-\delta$.

Then $\left(\Sigma_{t} \cap B_{\frac{\varepsilon}{K}}(O)\right)_{P_{t}}$ can be parametrized by

$$
X=\Phi\left(y_{1}, y_{2}, u\left(y_{1}, y_{2}, t\right)\right)
$$

for

$$
\sqrt{y_{1}^{2}+y_{2}^{2}}<r, y_{2} \geq 0,-\frac{\delta}{K^{2}} \leq t \leq \frac{\delta}{K^{2}} \iota,
$$

where $P_{t}$ is the normal trajectory of $P$ along the flow (i.e. $P_{t} \in \Sigma_{t}$ and $\partial_{t} P_{t} \perp$ $\left.T_{P_{t}} \Sigma_{t}\right)$ and $\Phi$ is the map in Definition 2.4.

Moreover, the function $u(y, t)$ satisfies

$$
\begin{gathered}
\partial_{t} u=g^{i j}(y, u, \nabla u) \partial_{i j}^{2} u+f(y, u, \nabla u), \\
\partial_{2} u\left(y_{1}, 0, t\right)=0
\end{gathered}
$$

where $g^{i j}(y, u, \nabla u)$ is the inverse of $g_{i j}(y, u, \nabla u)$, and $g_{i j}(y, u, \nabla u), f(y, u, \nabla u)$ are defined in (2.11), (2.13), respectively.

Furthermore, we have the following estimates:

$$
\begin{gathered}
K|u(y, t)|+|\nabla u(y, t)| \leq \varepsilon, \quad\left|\nabla^{2} u(y, t)\right| \lesssim K, \\
\left|g^{i j}(y, u, \nabla u)-\delta^{i j}\right| \leq \varepsilon, \quad\left[g^{i j}(y, u, \nabla u)\right]_{1, \text { spacial }} \lesssim K, \\
\left.g^{12}(y, u, \nabla u)\right|_{x_{2}=0}=0, \\
|f(y, u, \nabla u)| \lesssim K, \quad[f(y, u, \nabla u)]_{1, \text { spatial }} \lesssim K^{2},
\end{gathered}
$$

where $[\cdot]_{1, \text { spacial }}$ is the Lipschitz norm with respect to the spacial variable defined by

$$
[v]_{1, \text { spacial }}=\sup _{y \neq y^{\prime}} \frac{\left|v(y, t)-v\left(y^{\prime}, t\right)\right|}{\left|y-y^{\prime}\right|} .
$$


Proof. By Lemma 2.8 and the assumption regarding $P$ and $N_{\Sigma_{0}}(P)$, if $\delta>0$ is sufficiently small, the flow

$$
\left\{\left(\Sigma_{t} \cap B_{\frac{\delta}{K}}(O)\right)_{P_{t}}\right\}_{-\frac{\delta}{K^{2}} \leq t \leq \frac{\delta}{K^{2}} \iota}
$$

are graphs with small gradients with respect to the direction $(0,0,1)$. Using Definition 2.4 and (2.1), the preimage of the flow under $\Phi$ are also graphs with respect to the direction $(0,0,1)$ and

$$
N_{\Sigma_{t}} \cdot \partial_{3} \Phi>0 .
$$

Consequently, the flow can be parametrized by

$$
X(y, t)=\Phi(y, u(y, t))
$$

for some function $u(y, t)$ which is defined on $\sqrt{y_{1}^{2}+y_{2}^{2}}<\frac{\delta}{K}, y_{2} \geq 0,-\frac{\delta}{K^{2}} \leq t \leq$ $\frac{\delta}{K^{2}} \iota$ and satisfies

$$
K|u(y, t)|+|\nabla u(y, t)| \leq \varepsilon, \quad K^{-1}\left|\nabla^{2} u(y, t)\right| \lesssim 1 .
$$

Note that $\delta>0$ is chosen so that that the above conditions can be met.

Next, in order to get more information on $u(y, t)$, let's compute

$$
\begin{gathered}
\partial_{t} X=\partial_{t} u \partial_{3} \Phi(y, u) ; \\
\partial_{i} X=\partial_{i} \Phi(y, u)+\partial_{i} u \partial_{3} \Phi(y, u), \quad i \in\{1,2\} ; \\
D_{i} \partial_{j} X=\partial_{i j}^{2} \Phi+\partial_{i j}^{2} u \partial_{3} \Phi+\partial_{i} u \partial_{j 3}^{2} \Phi+\partial_{j} u \partial_{i 3}^{2} \Phi+\partial_{i} u \partial_{j} u \partial_{33}^{2} \Phi, \quad i, j \in\{1,2\} .
\end{gathered}
$$

Note that since $\Sigma_{t}$ is orthogonal to $\Gamma$ along the boundary and $N_{\Sigma_{t}} \cdot \partial_{2} X=0$, we have

$$
0=\left.N_{\Sigma_{t}} \cdot \nu\right|_{\partial \Sigma_{t}}=\left.N_{\Sigma_{t}} \cdot \partial_{2} \Phi\right|_{\partial \Sigma_{t}}=-\left.\partial_{2} u\left(y_{1}, 0, t\right) N_{\Sigma_{t}} \cdot \partial_{3} \Phi\right|_{\partial \Sigma_{t}}
$$

which, together with (2.10), yields (2.8). Thus $u(y, t)$ satisfies the homogeneous Neumann boundary condition. Moreover, the induced metric and the second fundamental form of $\Sigma_{t}$ are respectively given by

$$
\begin{gathered}
g_{\Sigma_{t}}: g_{i j}(y, u, \nabla u)=\partial_{i} X \cdot \partial_{j} X \\
=h_{i j}(y, u)+h_{i 3}(y, u) \partial_{j} u+h_{j 3}(y, u) \partial_{i} u+h_{33}(y, u) \partial_{i} u \partial_{j} u
\end{gathered}
$$

and

$$
\begin{gathered}
A_{\Sigma_{t}}: A_{i j}\left(y, u, \nabla u, \nabla^{2} u\right)=D_{i} \partial_{j} X \cdot N_{\Sigma_{t}} \\
=\partial_{3} \Phi \cdot N_{\Sigma_{t}}\left\{\Gamma_{i j}^{3}(y, u)+\partial_{i j}^{2} u+Q_{i j}(y, u, \nabla u)\right\}
\end{gathered}
$$

for $i, j \in\{1,2\}$, where $h_{i j}$ and $\Gamma_{i j}^{k}$ are defined in (2.3) and (2.4), respectively, and

$$
Q_{i j}(y, u, \nabla u)=\Gamma_{i 3}^{3}(y, u) \partial_{j} u+\Gamma_{j 3}^{3}(y, u) \partial_{i} u-\Gamma_{i j}^{k}(y, u) \partial_{k} u
$$

$+\Gamma_{33}^{3}(y, u) \partial_{i} u \partial_{j} u-\Gamma_{i 3}^{k}(y, u) \partial_{j} u \partial_{k} u-\Gamma_{j 3}^{k}(y, u) \partial_{i} u \partial_{k} u-\Gamma_{33}^{k}(y, u) \partial_{i} u \partial_{j} u \partial_{k} u$.

Note that by (2.5), (2.8) and (2.11), we get

$$
\left.g_{12}(y, u, \nabla u)\right|_{y_{2}=0}=0,
$$

from which, (2.9) follows immediately.

Using the MCF equation

$$
\partial_{t} X \cdot N_{\Sigma_{t}}=H_{\Sigma_{t}}=g^{i j}(y, u, \nabla u) A_{i j},
$$

we derive

$$
\partial_{t} u=g^{i j}(y, u, \nabla u) \partial_{i j}^{2} u+f(y, u, \nabla u)
$$


where

$$
f(y, u, \nabla u)=g^{i j}(y, u, \nabla u)\left\{\Gamma_{i j}^{3}(y, u)+Q_{i j}(y, u, \nabla u)\right\}
$$

By (2.1), (2.3), (2.4) and the $C^{2}$ estimate of $u(y, t)$, it's not hard to see that

$$
\left|g^{i j}(y, u, \nabla u)-\boldsymbol{\delta}^{i j}\right| \leq \varepsilon, \quad K^{-1}\left[g^{i j}(y, u, \nabla u)\right]_{1, \text { spacial }} \lesssim 1,
$$

and

$$
K^{-1}|f(y, u, \nabla u)|+K^{-2}[f(y, u, \nabla u)]_{1, \text { spacial }} \lesssim 1
$$

The following is the reflection principle for parabolic equations with homogeneous Neumann boundary condition. The proof is omitted since it is based on a simple calculation.

Lemma 2.10. (Reflection Principle)

Let $u(x, t) \in C^{2}\left(B_{r}^{+}(O) \times\left(-r^{2}, 0\right]\right)$ be a solution of

$$
\partial_{t} u=a^{i j}(x, t) \partial_{i j}^{2} u+f(x, t)
$$

where $r>0$ is a constant, $B_{r}^{+}(O)=\left\{\left(x_{1}, x_{2}\right) \mid \sqrt{x_{1}^{2}+x_{2}^{2}}<r, x_{2} \geq 0\right\}$ is the upper half ball in $\mathbb{R}^{2},\left\{a^{i j}(x, t)\right\}_{i, j \in\{1,2\}}$ and $f(x, t)$ are continuous functions on $B_{r}^{+}(O) \times\left(-r^{2}, 0\right], a^{12}(x, t)=a^{21}(x, t)$.

Suppose that

$$
\partial_{2} u\left(x_{1}, 0, t\right)=0 \quad \text { and } \quad a^{12}\left(x_{1}, 0, t\right)=0
$$

for $x_{1} \in(-r, r), t \in\left(-r^{2}, 0\right]$. Define the even extension of $u(x, t)$ as

$$
\bar{u}(x, t)=\left\{\begin{array}{l}
u(x, t), x_{2} \geq 0 \\
u(\tilde{x}, t), x_{2}<0
\end{array},\right.
$$

where $\left.\tilde{x}=\widetilde{\left(x_{1}, x_{2}\right.}\right)=\left(x_{1},-x_{2}\right)$ is the reflection of $x$ with respect to the line $\left\{x_{2}=0\right\}$ in $\mathbb{R}^{2}$. Then $\bar{u}(x, t) \in C^{2}\left(B_{r}(O) \times\left(-r^{2}, 0\right]\right)$ and it satisfies

$$
\partial_{t} \bar{u}=\bar{a}^{i j}(x, t) \partial_{i j}^{2} \bar{u}+\bar{f}(x, t)
$$

where

$$
\begin{gathered}
\bar{a}^{i j}(x, t)=\left\{\begin{array}{c}
a^{i j}(x, t), x_{2} \geq 0 \\
(-1)^{i+j} a^{i j}(\tilde{x}, t), x_{2}<0
\end{array}, \quad i, j \in\{1,2\} ;\right. \\
\bar{f}(x, t)=\left\{\begin{array}{c}
f(x, t), x_{2} \geq 0 \\
f(\tilde{x}, t), x_{2}<0
\end{array} .\right.
\end{gathered}
$$

Note that $\left\{\bar{a}^{i j}(x, t)\right\}_{i, j \in\{1,2\}}, \bar{f}(x, t)$ are continuous on $B_{r}(O) \times\left(-r^{2}, 0\right]$ and that $\bar{a}^{12}(x, t)=\bar{a}^{21}(x, t)$.

In the next lemma, we show how to use the given the spatial bounds and the comparison principle to derive the Hölder estimate with respect to the time variables (cf. $\mathrm{An}$ ).

Lemma 2.11. (Regularity in the Time Variable)

Let $u(x, t) \in C^{2}\left(B_{r}(O) \times\left(-r^{2}, 0\right]\right)$ satisfy

$$
\frac{|u(x, t)|}{r}+|\nabla u(x, t)|+r\left|\nabla^{2} u(x, t)\right| \lesssim 1,
$$

where $r>0$ is a constant and $B_{r}(O)$ is the open ball in $\mathbb{R}^{2}$ centered at $O$ with radius $r$. 
Suppose that $u(x, t)$ satisfies the equation

$$
\partial_{t} u=a^{i j}(x, t) \partial_{i j}^{2} u+f(x, t),
$$

where $\left\{a^{i j}(x, t)\right\}_{i, j \in\{1,2\}}$ and $f(x, t)$ are continuous functions satisfying

$$
\begin{gathered}
\frac{1}{2} \delta^{i j} \leq a^{i j}(x, t) \leq 2 \delta^{i j}, \quad a^{12}(x, t)=a^{21}(x, t), \quad r\left[a^{i j}\right]_{1, \text { spacial }} \lesssim 1, \\
r|f|+r^{2}[f]_{1, \text { spacial }} \lesssim 1
\end{gathered}
$$

on $B_{r}(O) \times\left(-r^{2}, 0\right]$ for $i, j \in\{1,2\}$, where $[\cdot]_{1, \text { spacial }}$ is the Lipschitz norm with respect to the spacial variable (see Lemma 2.9). Then we have

$$
\frac{\left|\nabla u(x, t)-\nabla u\left(x, t^{\prime}\right)\right|}{\left|t-t^{\prime}\right|^{\frac{1}{2}}} \lesssim \frac{1}{r}
$$

for $x \in B_{\frac{r}{4}}(O), 0<t-t^{\prime} \ll r^{2}$.

Proof. Fix $x_{0} \in B_{\frac{r}{4}}(O), t_{0}<0$ with $\left|t_{0}\right| \ll r^{2}$ (to be determined), and $k \in\{1,2\}$. Below we will show that

$$
\left|\partial_{k} u\left(x_{0}, t\right)-\partial_{k} u\left(x_{0}, t_{0}\right)\right| \lesssim \frac{\sqrt{t-t_{0}}}{r}
$$

for $t_{0}<t \leq 0$.

For each $0<h<\frac{r}{4}$, the difference quotient of $u(x, t)$ is defined by

$$
\triangle_{k}^{h} u(x, t)=\frac{1}{h}\left(u\left(x+h e_{k}, t\right)-u(x, t)\right) .
$$

Using the equation of $u(x, t)$ and the assumption that

$$
|\nabla u(x, t)|+r\left|\nabla^{2} u\right|+r\left[a^{i j}\right]_{1, \text { spacial }}+r^{2}[f]_{1, \text { spacial }} \lesssim 1,
$$

there is $C>0$ so that

$$
\left|\triangle_{k}^{h} u(x, t)\right| \leq \frac{C}{4}
$$

and

$$
\begin{gathered}
\partial_{t} \triangle_{k}^{h} u=a^{i j}\left(x+h e_{k}, t\right) \partial_{i j}^{2} \triangle_{k}^{h} u+\left(\triangle_{k}^{h} a^{i j}\right) \partial_{i j}^{2} u+\triangle_{k}^{h} f \\
\leq a^{i j}\left(x+h e_{k}, t\right) \partial_{i j}^{2} \triangle_{k}^{h} u+\frac{C}{r^{2}} .
\end{gathered}
$$

By the mean value theorem, we may assume that for the same constant $C>0$, there holds

$$
\begin{gathered}
\triangle_{k}^{h} u\left(x, t_{0}\right) \leq \triangle_{k}^{h} u\left(x_{0}, t_{0}\right)+\frac{C}{r}\left|x-x_{0}\right| \\
\leq \triangle_{k}^{h} u\left(x_{0}, t_{0}\right)+\varepsilon+\frac{1}{4 \varepsilon}\left(\frac{C}{r}\right)^{2}\left|x-x_{0}\right|^{2} \quad \forall \varepsilon>0,
\end{gathered}
$$

in which we use Young's inequality to get the second line.

For each $\varepsilon>0$, consider an auxiliary function

$$
v_{\varepsilon}(x, t):=\triangle_{k}^{h} u\left(x_{0}, t_{0}\right)+\varepsilon+\frac{1}{4 \varepsilon}\left(\frac{C}{r}\right)^{2}\left|x-x_{0}\right|^{2}+\left(\frac{2}{\varepsilon}\left(\frac{C}{r}\right)^{2}+\frac{C}{r^{2}}\right)\left(t-t_{0}\right) \text {. }
$$


Obviously, by (2.16), we have $v_{\varepsilon}\left(x, t_{0}\right) \geq \triangle_{k}^{h} u\left(x, t_{0}\right)$ for $x \in B_{\frac{r}{2}}\left(x_{0}\right)$. Also, (2.15) and the condition that $a^{i j} \leq 2 \delta^{i j}$ yield

$$
\left(\partial_{t}-a^{i j}\left(x+h e_{k}, t\right) \partial_{i j}^{2}\right) v_{\varepsilon} \geq \frac{C}{r^{2}} \geq\left(\partial_{t}-a^{i j}\left(x+h e_{k}, t\right) \partial_{i j}^{2}\right) \triangle_{k}^{h} u .
$$

In addition, by (2.14), for any $(x, t)$ satisfying $\left|x-x_{0}\right|=\frac{r}{2}$ and $t_{0} \leq t \leq 0$, we have

$$
\begin{gathered}
v_{\varepsilon}(x, t) \geq \triangle_{k}^{h} u\left(x_{0}, t_{0}\right)+\varepsilon+\frac{1}{4 \varepsilon}\left(\frac{C}{r}\right)^{2}\left|x-x_{0}\right|^{2} \geq \triangle_{k}^{h} u\left(x_{0}, t_{0}\right)+\frac{C}{r}\left|x-x_{0}\right| \\
\geq-\frac{C}{4}+\frac{C}{r}\left|x-x_{0}\right|=\frac{C}{4} \geq \triangle_{k}^{h} u(x, t) .
\end{gathered}
$$

The comparison principle for parabolic equations then gives

$$
\triangle_{k}^{h} u(x, t) \leq v_{\varepsilon}(x, t)
$$

for $\left|x-x_{0}\right| \leq \frac{r}{2}, t_{0} \leq t \leq 0$. In particular, we get

$$
\triangle_{k}^{h} u\left(x_{0}, t\right) \leq \triangle_{k}^{h} u\left(x_{0}, t_{0}\right)+\varepsilon+\left(\frac{C}{r^{2}}+\frac{1}{\varepsilon}\left(\frac{C}{r}\right)^{2}\right)\left(t-t_{0}\right)
$$

for any $t \in\left[t_{0}, 0\right], \varepsilon>0$.

For each $t \in\left(t_{0}, t_{0}+\frac{r^{2}}{C^{2}}\right]$, choose $\varepsilon=\frac{C}{r} \sqrt{t-t_{0}}$, where $C>0$ is the aforementioned constant. By (2.17), we get

$$
\triangle_{k}^{h} u\left(x_{0}, t\right)-\triangle_{k}^{h} u\left(x_{0}, t_{0}\right) \leq 2 \frac{C}{r} \sqrt{t-t_{0}}+\frac{C}{r^{2}}\left(t-t_{0}\right) \leq\left(2 C+\frac{1}{C}\right) \frac{\sqrt{t-t_{0}}}{r} .
$$

As $h \searrow 0$, we get

$$
\partial_{k} u\left(x_{0}, t\right)-\partial_{k} u\left(x_{0}, t_{0}\right) \leq\left(2 C+\frac{1}{C}\right) \frac{\sqrt{t-t_{0}}}{r} .
$$

Lastly, choose $-\frac{r^{2}}{C^{2}} \leq t_{0}<0$ so that the above hold for $t \in\left(t_{0}, 0\right]$.

Replacing $u$ by $-u$ and following the same procedure gives

$$
-\partial_{k} u\left(x_{0}, t\right)+\partial_{k} u\left(x_{0}, t_{0}\right) \leq\left(2 C+\frac{1}{C}\right) \frac{\sqrt{t-t_{0}}}{r}
$$

for $t_{0}<t \leq 0$.

With the help of Lemma 2.10 and Lemma 2.11, we can improve Lemma 2.9 as follows.

Proposition 2.12. ( $C^{2,1}$ Estimates for $M C F$ with Free Boundary)

The function $u(y, t)$ in Lemma 2.9 satisfies

$$
K|u(y, t)| \ll 1, \quad\left[\nabla^{2} u\right]_{1}+\left[\partial_{t} u\right]_{1} \lesssim K^{2}
$$

for $|x|+|t|^{\frac{1}{2}} \ll \frac{1}{K}$, where $[\cdot]_{1}$ is the "parabolic" Lipschitz norm defined by

$$
[v]_{1}=\sup _{\left|y-y^{\prime}\right|+\left|t-t^{\prime}\right|>0} \frac{\left|v(y, t)-v\left(y^{\prime}, t^{\prime}\right)\right|}{\left|y-y^{\prime}\right|+\left|t-t^{\prime}\right|^{\frac{1}{2}}}
$$


Proof. By (2.8) and (2.9), Lemma 2.10 implies that the even extension $\bar{u}(y, t)$ of $u(y, t)$ across $\left\{y_{2}=0\right\}$ satisfies

$$
\partial_{t} \bar{u}=\bar{g}^{i j}(y, t) \partial_{i j}^{2} \bar{u}+\bar{f}(x, t),
$$

where

$$
\begin{gathered}
\bar{g}^{i j}(y, t)=\left\{\begin{array}{c}
g^{i j}(y, u(y, t), \nabla u(x, t)), y_{2} \geq 0 \\
(-1)^{i+j} g^{i j}(\tilde{y}, u(\tilde{y}, t), \nabla u(\tilde{y}, t)), y_{2}<0
\end{array}, \quad i, j \in\{1,2\} ;\right. \\
\bar{f}(y, t)=\left\{\begin{array}{c}
f(y, u(y, t), \nabla u(y, t)), y_{2} \geq 0 \\
f(\tilde{y}, u(\tilde{y}, t), \nabla u(\tilde{y}, t)), y_{2}<0
\end{array} .\right.
\end{gathered}
$$

Moreover, we have

$$
\begin{gathered}
K|\bar{u}(y, t)|+|\nabla \bar{u}(y, t)|+K^{-1}\left|\nabla^{2} \bar{u}(y, t)\right| \lesssim 1, \\
\left|\bar{g}^{i j}-\delta^{i j}\right| \ll 1, \quad\left[\bar{g}^{i j}\right]_{1, \text { spacial }} \lesssim K, \\
|f| \lesssim K, \quad[f]_{1, \text { spacial }} \lesssim K^{2} .
\end{gathered}
$$

which, by Lemma 2.11, implies that

$$
\left|\nabla \bar{u}(y, t)-\nabla \bar{u}\left(y^{\prime}, t^{\prime}\right)\right| \lesssim K\left(\left|y-y^{\prime}\right|+\left|t-t^{\prime}\right|^{\frac{1}{2}}\right) .
$$

By definitions of $\bar{g}^{i j}(y, t)$ and $\bar{f}(y, t)$ (and interpolation inequalities), we then get

$$
\begin{gathered}
\left|\bar{g}^{i j}(y, t)-\bar{g}^{i j}\left(y^{\prime}, t^{\prime}\right)\right| \lesssim K\left(\left|y-y^{\prime}\right|+\left|t-t^{\prime}\right|^{\frac{1}{2}}\right), \\
\left|\bar{f}(y, t)-\bar{f}\left(y^{\prime}, t^{\prime}\right)\right| \lesssim K^{2}\left(\left|y-y^{\prime}\right|+\left|t-t^{\prime}\right|^{\frac{1}{2}}\right) .
\end{gathered}
$$

The conclusion follows immediately by applying Schauder estimates to the equation of $\bar{u}(y, t)$.

Lastly, we conclude this section with the fourth topic: the compactness of the space of MCF with free boundary. Let's first give a definition of the topology of the space of MCF with free boundary.

Definition 2.13. (Topology of the Space of MCF with Free Boundary)

Fix $\iota \in\{0,1\}$. Let $\left\{\left\{\Sigma_{t}^{i}\right\}_{a<t<b}\right\}_{i \in \mathbb{N}}$ be a sequence of MCF which moves freely in $\left\{U_{i} \subset \mathbb{R}^{3}\right\}_{i \in \mathbb{N}}$ respectively. Also, let $\left\{\Sigma_{t}\right\}_{a<t<b}$ be a properly embedded $C^{2}$ MCF moving freely in $U \subset \mathbb{R}^{3}$. We say that $\left\{\Sigma_{t}^{i}\right\}$ converges $^{\iota}$ to $\left\{\Sigma_{t}\right\}$ with finite multiplicity as $i \rightarrow \infty$ if the following hold.

(1) $U_{i}$ converges to $U$ in the sense that $U$ consists of all limit points of $U_{i}$ and $\partial U_{i}=\Gamma_{i} \stackrel{C^{3}}{\rightarrow} \Gamma=\partial U$ as $i \rightarrow \infty$

(2) For each $t_{*} \in(a, b), \Sigma_{t_{*}}$ consists of all limit points of $\left\{\Sigma_{t_{*}}^{i}\right\}_{i \in \mathbb{N}}$. In addition, for every $P \in \Sigma_{t_{*}}$, there exist $r>0$ and $m \in \mathbb{N}$ so that

- There is a $C^{2}$ diffeomorphism $\Phi$ from an open neighborhood of $O$ in $\mathbb{R}^{3}$ onto $B_{r}(P)$ so that $\Phi^{-1}\left(\Sigma_{t} \cap B_{r}(P)\right)$ is a graph of a function $u(y, t)$ defined for $y \in \Omega \subset \mathbb{R}^{2}, t \in\left[t_{*}-r^{2}, t_{*}+\iota r^{2}\right]$, where

$$
\Omega=\left\{\begin{array}{cl}
\left\{\left(y_{1}, y_{2}\right) \mid \sqrt{y_{1}^{2}+y_{2}^{2}}<r\right\}, \quad \text { if } \quad P \in U \\
\left\{\left(y_{1}, y_{2}\right) \mid \sqrt{y_{1}^{2}+y_{2}^{2}}<r, y_{2} \geq 0\right\}, \quad \text { if } P \in \Gamma
\end{array} .\right.
$$

Note that if $P \in \Gamma$, we require $\Gamma \cap B_{r}(P)=\Phi\left\{y_{2}=0\right\}$ and $\Phi^{-1}\left(\Sigma_{t} \cap B_{r}(P)\right)$ meets $\left\{y_{2}=0\right\}$ orthogonally. 
- For each $i \gg 1$, there is a $C^{2}$ diffeomorphism $\Phi_{i}$ from an open neighborhood of $O$ in $\mathbb{R}^{3}$ onto $B_{r}(P)$ so that $\Phi_{i}^{-1}\left(\Sigma_{t}^{i} \cap B_{r}(P)\right)$ consists of $m$ graphs of functions

$$
\left\{u_{i}^{1}(y, t), \cdots, u_{i}^{m}(y, t)\right\}
$$

defined for $y \in \Omega, t_{*}-r^{2} \leq t \leq t_{*}+\iota r^{2}$; moreover, if $P \in \Gamma, \Gamma_{i} \cap$ $B_{r}(P)=\Phi_{i}\left\{y_{2}=0\right\}$ and $\Phi_{i}^{-1}\left(\Sigma_{t}^{i} \cap B_{r}(P)\right)$ meets $\left\{y_{2}=0\right\}$ orthogonally. Furthermore, as $i \rightarrow \infty$ we have

$$
\begin{gathered}
\Phi_{i} \stackrel{C^{2}}{\rightarrow} \Phi, \\
u_{i}^{j} \stackrel{C^{2}}{\rightarrow} u \quad \forall j \in\{1, \cdots, m\} .
\end{gathered}
$$

Before coming to the compactness theorem, let's introduce the following notation in order to simplify the notation in the proof.

Definition 2.14. (Representation of a Local Graph)

Given a point $Q \in \mathbb{R}^{3}$, an orthonormal basis $\omega=\left\{e_{1}, e_{2}, e_{3}\right\}$ in $\mathbb{R}^{3}$, and a function $u: \Omega \subset \mathbb{R}^{2} \rightarrow \mathbb{R}$, let $[Q, \omega, u]$ be the graph of $u$ with respect to the orientation $\omega$ and centered at $Q$. That is,

$$
[Q, \omega, u]=\left\{X\left(y_{1}, y_{2}\right)=Q+y_{1} e_{1}+y_{2} e_{2}+u\left(y_{1}, y_{2}\right) e_{3} \mid\left(y_{1}, y_{2}\right) \in \Omega\right\} .
$$

What follows is the the compactness theorem that will be used frequently in the later sections (cf. [PR]).

Proposition 2.15. (Compactness of the Space of MCF with Free Boundary)

Fix $\iota \in\{0,1\}$. Let $\left\{\left\{\Sigma_{t}^{i}\right\}_{a<t<b}\right\}_{i \in \mathbb{N}}$ be a sequence of connected, properly embedded $C^{2}$ MCF moving freely in $\left\{U_{i} \subset \mathbb{R}^{3}\right\}_{i \in \mathbb{N}}$ respectively. Suppose that

- There is $\kappa>0$ so that each $\Gamma_{i}=\partial U_{i}$ is mean convex (i.e. the mean curvature vector of $\Gamma_{i}$ points toward $U_{i}$ ) and satisfies the $\kappa-$ graph condition. Note that by passing to a subsequence, we may assume that $U_{i} \rightarrow U$ as $i \rightarrow \infty$ (in the sense that $U$ consists of all limit points of $U_{i}$ and $\Gamma_{i}=$ $\left.\partial U_{i} \stackrel{C^{3}}{\rightarrow} \Gamma=\partial U\right)$

- The areas and second fundamental forms of the flows are locally uniformly bounded ${ }^{t}$. Namely, for each $t_{*} \in(a, b)$ and $P \in \mathbb{R}^{3}$, there is $r>0$ so that for every $i \in \mathbb{N}$, there holds

$$
\sup _{t_{*}-r^{2} \leq t \leq t_{*}+\iota r^{2}}\left(\mathcal{H}^{2}\left(\Sigma_{t}^{i} \cap B_{r}(P)\right)+\left\|A_{\Sigma_{t}^{i}}\right\|_{L^{\infty}\left(B_{r}(P)\right)}\right) \leq C\left(P, t_{*}, r\right)<\infty ;
$$

- There is a time $t_{0}$ so that every subsequence of $\left\{\Sigma_{t_{0}}^{i}\right\}_{i \in \mathbb{N}}$ has limit points which are not in $\Gamma$.

Then $\left\{\Sigma_{t}^{i}\right\}_{t \geq t_{0}}$ converges ${ }^{l}$ to a MCF moving freely in $U$ as $i \rightarrow \infty$ (in the sense of Definition 2.13).

Proof. Due to the the uniform $\kappa$-graph condition (see Definition 2.3), we may assume, by passing to a subsequence, that $U_{i} \rightarrow U$ as $i \rightarrow \infty$. Moreover, the convergence implies that $\Gamma$ is mean convex and satisfies the $\kappa$-graph condition as well.

Fix $P \in U \cup \Gamma$ and $t_{*} \in \mathbb{R}$. If $P$ is not a limit point of $\left\{\sum_{t_{*}}^{i}\right\}_{i \in \mathbb{N}}$, by the local bound on the second fundamental form (which controls the normal speed of the (low), we may assume, by passing to a subsequence, that there is $r>0$ so that 
$\Sigma_{t}^{i} \cap B_{r}(P)=\emptyset$ for $t_{*}-r^{2} \leq t \leq t_{*}+\iota r^{2}, i \in \mathbb{N}$. In case $P$ is a limit point of $\left\{\Sigma_{t_{*}}^{i}\right\}_{i \in \mathbb{N}}$, below we divide into two cases to consider:

- Case 1: $P \in U$;

- Case 2: $P \in \Gamma$.

Case $1(P \in U)$ :

By passing to a subsequence, we may assume that there exist $P_{i} \in \Sigma_{t_{*}}^{i} \cap U_{i}$ which converge to $P$ as $i \rightarrow \infty$. The properly embeddedness and the uniform bound on the second fundamental forms imply that there exists $r>0$ with the following property. For each $i \in \mathbb{N}$, there are a number of time-dependent local graphs

$$
\left\{\left[Q_{i}^{j}, \omega_{i}^{j}, u_{i}^{j}: B_{3 r}(O) \times\left[t_{*}-9 r^{2}, t_{*}+9 \iota r^{2}\right] \rightarrow \mathbb{R}\right]\right\}_{j=1, \cdots, m_{i}}
$$

whose union covers $\left\{\Sigma_{t}^{i} \cap B_{r}\left(P_{i}\right)\right\}_{t_{*}-r^{2} \leq t \leq t_{*}+\iota r^{2}}$ and which are mutually disjoint in $B_{2 r}\left(P_{i}\right)$ for $t_{*}-4 r^{2} \leq t \leq t_{*}+4 \iota r^{2}$. The uniform bound for the local areas yields that

$$
\sup _{i \in \mathbb{N}} m_{i} \leq C\left(P, t_{*}, r\right),
$$

so we may assume, by passing to a subsequence, that $m_{i}=m$ for $i \in \mathbb{N}$. By the smooth estimate for MCF (see $[\mathrm{E}]$ for analogous results of Proposition 2.12 for the interior case), there holds

$$
\max _{1 \leq j \leq m}\left\|u_{i}^{j}\right\|_{C^{2,1}} \leq C\left(P, t_{*}, r\right) .
$$

It follows, by passing to a subsequence, that

$$
Q_{i}^{j} \rightarrow Q^{j}, \quad \omega_{i}^{j} \rightarrow \omega^{j}, \quad u_{i}^{j} \stackrel{C^{2}}{\rightarrow} u \quad \forall j \in\{1, \cdots, m\}
$$

as $i \rightarrow \infty$. Clearly, each limiting local graph $\left[Q^{j}, \omega^{j}, u^{j}\right]$ is a solution of MCF. Furthermore, every two limiting local graphs must be either disjoint or identical by the strong maximum principle.

Case $2(P \in \Gamma)$ :

Passing to a subsequence, there are $P_{i} \in \Sigma_{t_{*}}^{i}$ so that $P_{i} \rightarrow P$ as $i \rightarrow \infty$. Let $\stackrel{\circ}{P}_{i}$ be the closest point on $\Gamma_{i}$ to $P_{i}$, then we have $\left|\stackrel{\circ}{P}_{i}-P_{i}\right| \rightarrow 0$ as $i \rightarrow \infty$ (since $P \in \Gamma$ and $\Gamma_{i} \rightarrow \Gamma$ ). By the $\kappa$-graph condition, the uniform bound on the second fundamental forms and Lemma 2.9, there exists $r>0$ with the following properties. For each $i \in \mathbb{N}$, there is a diffeomorphism $\Phi_{i}$ (see Definition 2.4) which maps from a neighborhood of $O$ in $\mathbb{R}^{3}$ onto $B_{3 r}\left(\stackrel{\circ}{P}_{i}\right)$ and

$$
U_{i} \cap B_{3 r}\left(\stackrel{\circ}{P}_{i}\right)=\Phi_{i}\left\{y_{2}>0\right\}, \quad \Gamma_{i} \cap B_{3 r}\left(\stackrel{\circ}{P}_{i}\right)=\Phi_{i}\left\{y_{2}=0\right\} ;
$$

moreover, there are a number of time-dependent local graphs $\left\{\left[Q_{i}^{j}, \omega_{i}^{j}, u_{i}^{j}\right]\right\}_{j=1, \cdots, m_{i}+m_{i}^{\prime}}$, whose union covers $\left\{\Phi_{i}^{-1}\left(\Sigma_{t}^{i} \cap B_{r}\left(P_{i}\right)\right)\right\}_{t_{*}-r^{2} \leq t \leq t_{*}+\iota r^{2}}$ and which are mutually disjoint in $B_{2 r}(O)$ for $t_{*}-4 r^{2} \leq t \leq t_{*}+4 \iota r^{2}$. The number $m_{i}$ and $m_{i}^{\prime}$ means the following:

- For $j \in\left\{1, \cdots, m_{i}\right\}$, the function $u_{i}^{j}(x, t)$ is defined on $B_{3 r}^{+}(O) \times\left[t_{*}-9 r^{2}, t_{*}+9 \iota r^{2}\right]$ and $\Phi_{i}\left[Q_{i}^{j}, \omega_{i}^{j}, u_{i}^{j}\right]$ meets $\Gamma_{i}$ orthogonally for $j \in\left\{1, \cdots, m_{i}\right\}$; 
- For $j \in\left\{m_{i}+1, \cdots, m_{i}+m_{i}^{\prime}\right\}$, the function $u_{i}^{j}(x, t)$ is defined on $B_{3 r}(O) \times$ $\left[t_{*}-9 r^{2}, t_{*}+9 \iota r^{2}\right]$ and $\Phi_{i}\left[Q_{i}^{j}, \omega_{i}^{j}, u_{i}^{j}\right]$ is contained in $U_{i}$.

The uniform bound on areas yields

$$
\sup _{i \in \mathbb{N}}\left(m_{i}+m_{i}^{\prime}\right) \leq C\left(P, t_{*}, r, \kappa\right),
$$

so, by passing to a subsequence, we may assume that $m_{i}=m$ and $m_{i}^{\prime}=m^{\prime}$ for $i \in \mathbb{N}$. In addition, Proposition 2.12 implies

$$
\max _{1 \leq j \leq m+m^{\prime}}\left\|u_{i}^{j}\right\|_{C^{2,1}} \leq C\left(P, t_{*}, r, \kappa\right) .
$$

It follows, by passing to a subsequence, that $\Phi_{i} \stackrel{C^{2}}{\rightarrow} \Phi$ and

$$
Q_{i}^{j} \rightarrow Q^{j}, \quad \omega_{i}^{j} \rightarrow \omega^{j}, \quad u_{i}^{j} \stackrel{C^{2}}{\rightarrow} u^{j}, \quad \forall j \in\left\{1, \cdots, m+m^{\prime}\right\}
$$

as $i \rightarrow \infty$. By the convergence, $\Phi$ is a local diffeomorphism from a neighborhood of $O$ in $\mathbb{R}^{3}$ to $B_{2 r}(P)$ and

$$
\begin{aligned}
& \Phi^{-1}\left(U \cap B_{2 r}(P)\right) \subset\left\{\left(y_{1}, y_{2}, y_{3}\right) \mid y_{2}>0\right\}, \\
& \Phi^{-1}\left(\Gamma \cap B_{2 r}(P)\right) \subset\left\{\left(y_{1}, y_{2}, y_{3}\right) \mid y_{2}=0\right\} .
\end{aligned}
$$

By the comparison principle (more precisely, using (2.7), Lemma 2.10 and [Wa]), every two limiting graphs must be either disjoint or identical. In addition, each $\Phi\left[Q^{j}, \omega^{j}, u^{j}\right]$ is clearly a solution of MCF. For $j \in\{1, \cdots, m\}, \Phi\left[Q^{j}, \omega^{j}, u^{j}\right]$ meets $\Gamma$ orthogonally. For $j \in\left\{m+1, \cdots, m+m^{\prime}\right\}$, due to the strong maximum principle and the mean convexity of $\Gamma$, either $\Phi\left[Q^{j}, \omega^{j}, u^{j}\right]$ and $\Gamma$ are disjoint, or $\Phi\left[Q^{j}, \omega^{j}, u^{j}\right] \subset \Gamma$.

Lastly, take a countable dense subset $\left\{\left(P_{k}, t_{k}\right)\right\}_{k \in \mathbb{N}}$ of $(U \cup \Gamma) \times\left[t_{0}, \infty\right)$. Applying the above argument for the sequence of points one by one successively and using Cantor's diagonal argument, we can extract a subsequence as claimed. Note that by the above argument, if the limiting flow $\left\{\Sigma_{t}\right\}$ intersects $\Gamma$ at interior points for $t_{1} \geq t_{0}$, then $\Sigma_{t} \subset \Gamma$ by the strong maximum principle for all $t \in\left[t_{0}, t_{1}\right]$, which contradicts with the assumption that $\Sigma_{t_{0}}$ is not contained in $\Gamma$.

\section{Area Ratio and Curvature Estimates for MCF}

In this section we follow closely the ideas in $[\mathrm{LW}$ to estimate the area ratio and second fundamental form of MCF with free boundary and uniformly bounded mean curvature. It consists of the following two parts.

(1) The area ratio of a surface in a sufficiently small ball (which would be modified near the boundary, see Lemma 3.1 for instance) stays close to one along MCF with free boundary and uniformly bounded mean curvature (see Proposition 3.5).

(2) Smallness of the $L^{2}$ norm of the initial second fundamental form yields the bound on the second fundamental form for MCF with free boundary and uniformly bounded mean curvature (see Proposition 3.10).

The proof of the first part proceeds as follows. We first show that the modified area ratio for the initial surface $\Sigma_{0}$ is close to one, provided that the radius is sufficiently small (depending on the curvatures of $\Sigma$ and the boundary support surface $\Gamma$ ). Then we show that the area of $\Sigma_{t}$ change slightly (forward and backward) in time if the mean curvature stays uniformly bounded. Lastly, by appealing to the monotonicity 
of area ratio for each $\Sigma_{t}$, we show that the modified area ratio stays close to one along the flow.

Recall that in (2.6), we denote by $(\Sigma)_{P}$ the path-connected component of a surface $\Sigma$ containing $P$. Also, $\tilde{B}_{r}(P)$ stands for the reflection of $B_{r}(P) \backslash U$ with respect to $\Gamma$ (see Definition 2.1).

Lemma 3.1. Given $\varepsilon>0$, there exists $\delta>0$ with the following property. Let $\Sigma$ be a properly embedded $C^{2}$ surface in $U \subset \mathbb{R}^{3}$ which meets $\Gamma=\partial U$ orthogonally. Suppose that

- Either $B_{1}(O) \subset U$, or $O \in \Gamma$ and $\Gamma$ satisfies the $\kappa$-graph condition for some $0<\kappa \leq 1$;

- There is $K \geq 1$ so that $\left(\Sigma \cap B_{\frac{\delta}{K}}(P)\right)_{P}$ is a $\delta$-Lipschitz graph for any $P \in \Sigma \cap B_{\frac{1}{2}}(O)$ (which holds, for instance, when $\left.\left\|A_{\Sigma}\right\|_{L^{\infty}\left(B_{1}(O)\right)} \leq K\right)$.

Then for any $P \in \Sigma \cap B_{\delta}(O)$ and $\rho \in\left[0, \frac{\delta}{K}\right)$, we have

$$
\frac{\mathcal{H}^{2}\left(\Sigma \cap B_{\rho}(P)\right)_{P}+\mathcal{H}^{2}\left(\left(\Sigma \cap B_{\rho}(P)\right)_{P} \cap \tilde{B}_{\rho}(P)\right)}{\pi \rho^{2}} \leq 1+\varepsilon .
$$

Proof. Fix $P \in \Sigma$ and $\rho>0$. If $\rho \leq d_{\Gamma}(P)$, then $\tilde{B}_{\rho}(P)=\emptyset$ (see Definition 2.1) and the conclusion follows directly from the small gradient graph condition. In the case when $\rho>d_{\Gamma}(P)$, one can first apply the map $\Phi$ in Definition 2.4 to pull back $\Sigma$ to a half-space and then use (2.1), (2.2), Remark 2.2 and the small gradient graph condition to get the conclusion.

Below we show that the area changes slightly along MCF within a time which is inversely proportional to the mean curvature.

Lemma 3.2. Given $\varepsilon>0$, there exists $\delta>0$ with the following property. Let $\left\{\Sigma_{t}\right\}_{a \leq t \leq b}$ be a properly embedded $C^{2} M C F$ which moves freely in $U \subset \mathbb{R}^{3}$ and has the following parametrization:

$$
X_{t}=X(\cdot, t): M^{2} \times[a, b] \rightarrow U \subset \mathbb{R}^{3}, \quad \partial_{t} X_{t}=\vec{H}_{\Sigma_{t}},
$$

where $a \leq 0 \leq b$ are constants. Suppose that

- Either $B_{1}(O) \subset U$, or $O \in \Gamma=\partial U$ and $\Gamma$ satisfies the $\kappa$-graph condition for some $0<\kappa \leq 1$;

- There is $0<\Lambda \leq 1$ so that $\sup _{a \leq t \leq b}\left\|H_{\Sigma_{t}}\right\|_{L^{\infty}\left(B_{1}(O)\right)} \leq \Lambda$.

Then for any $\rho \in(0, \delta], t \in[\breve{a}, \breve{b}]$ and $P \in \Sigma_{t} \cap B_{\delta}(O)$, we have

$$
\begin{gathered}
\frac{\mathcal{H}^{2}\left(\Sigma_{t} \cap B_{\rho}(P)\right)_{P}}{\pi \rho^{2}} \leq e^{\Lambda^{2}|t|}\left(1+\frac{2 \Lambda|t|}{\rho}\right)^{2} \frac{\mathcal{H}^{2}\left(\Sigma_{0} \cap B_{\rho+2 \Lambda|t|}\left(P_{0}\right)\right)_{P_{0}}}{\pi(\rho+2 \Lambda|t|)^{2}} \\
\frac{\mathcal{H}^{2}\left(\left(\Sigma_{t} \cap B_{\rho}(P)\right)_{P} \cap \tilde{B}_{\rho}(P)\right)}{\pi \rho^{2}} \\
\leq e^{\Lambda^{2}|t|}\left(1+\frac{2(1+\varepsilon) \Lambda|t|}{\rho}\right)^{2} \frac{\mathcal{H}^{2}\left(\left(\Sigma_{0} \cap B_{\rho+2(1+\varepsilon) \Lambda|t|}\left(P_{0}\right)\right)_{P_{0}} \cap \tilde{B}_{\rho+2(1+\varepsilon) \Lambda|t|}\left(P_{0}\right)\right)}{\pi(\rho+2(1+\varepsilon) \Lambda|t|)^{2}}
\end{gathered}
$$

where

$$
\breve{a}=\max \left\{a,-\frac{\delta}{\Lambda}\right\}, \quad \breve{b}=\min \left\{b, \frac{\delta}{\Lambda}\right\}, \quad P_{0}=X_{0} \circ X_{t}^{-1}(P)
$$


Proof. Given $t$ and $P \in \Sigma_{t}$, let $P_{0}=X_{0} \circ X_{t}^{-1}(P)$. Since

$$
\partial_{t}|X(p, t)-X(p, 0)| \leq \Lambda, \quad \partial_{t}|X(p, t)-X(q, t)| \leq 2 \Lambda
$$

for any $p, q \in M$, we get $\left|P-P_{0}\right| \leq \Lambda|t|$ and

$$
X_{t}^{-1}\left(\Sigma_{t} \cap B_{\rho}(P)\right)_{P} \subset X_{0}^{-1}\left(\Sigma_{0} \cap B_{\rho+2 \Lambda|t|}\left(P_{0}\right)\right)_{P_{0}} .
$$

Moreover, by the evolution formula $\partial_{t} d \mu_{t}=-H_{\Sigma_{t}}^{2} d \mu_{t}$, where $d \mu_{t}$ is the induced measure of $\Sigma_{t}$ on $M$, it follows that

$$
\begin{gathered}
\mathcal{H}^{2}\left(\Sigma_{t} \cap B_{R}(P)\right)_{P} \leq \mathcal{H}^{2}\left(X_{t} \circ X_{0}^{-1}\left(\Sigma_{0} \cap B_{R+2 \Lambda|t|}\left(P_{0}\right)\right)_{P_{0}}\right) \\
\leq e^{\Lambda^{2}|t|} \mathcal{H}^{2}\left(\Sigma_{0} \cap B_{R+2 \Lambda|t|}\left(P_{0}\right)_{P_{0}}\right)
\end{gathered}
$$

This proves the first inequality.

Likewise, for the second inequality, it suffices to show that

$X_{t}^{-1}\left(\left(\Sigma_{t} \cap B_{\rho}(P)\right)_{P} \cap \tilde{B}_{\rho}(P)\right) \subset X_{0}^{-1}\left(\left(\Sigma_{0} \cap B_{\rho+2(1+\varepsilon) \Lambda|t|}\left(P_{0}\right)\right)_{P_{0}} \cap \tilde{B}_{\rho+2(1+\varepsilon) \Lambda|t|}\left(P_{0}\right)\right)$.

To see that, fix $Q \in\left(\Sigma_{t} \cap B_{\rho}(P)\right)_{P} \cap \tilde{B}_{\rho}(P)$ and let $Q_{0}=X_{0} \circ X_{t}^{-1}(Q)$. Notice that $\tilde{Q} \in B_{\rho}(P)$ (see Definition 2.1) and $Q_{0} \in\left(\Sigma_{0} \cap B_{\rho+2 \Lambda|t|}\left(P_{0}\right)\right)_{P_{0}}$. Given $\varepsilon>0$, by (2.1) and (2.2), there is $\delta>0$ so that

$$
\left|\widetilde{Q_{0}}-\tilde{Q}\right| \leq(1+\varepsilon)\left|Q_{0}-Q\right|
$$

as long as $P \in B_{\delta}(O), 0<\rho \leq \delta$ and $\Lambda|t| \leq \delta$. It follows that

$$
\widetilde{Q_{0}} \in B_{\rho+(1+\varepsilon) \Lambda|t|}(P) \subset B_{\rho+2(1+\varepsilon) \Lambda|t|}\left(P_{0}\right),
$$

which, by Definition 2.1, yields $Q_{0} \in \tilde{B}_{\rho+2(1+\varepsilon) \Lambda|t|}\left(P_{0}\right)$.

The following is an immediate consequence of Lemma 3.2

Corollary 3.3. Given $\varepsilon>0$, there exists $\delta>0$ with the following property. Let $\left\{\Sigma_{t}\right\}_{a \leq t \leq b}$ be as in Lemma [3.2. Then for any $\rho \in(0, \delta], t \in[\breve{a}, \breve{b}]$ and $P \in$ $\Sigma_{t} \cap B_{\delta}(O)$, there holds

$$
\begin{gathered}
\frac{\mathcal{H}^{2}\left(\Sigma_{t} \cap B_{\rho}(P)\right)_{P}+\mathcal{H}^{2}\left(\left(\Sigma_{t} \cap B_{\rho}(P)\right)_{P} \cap \tilde{B}_{\rho}(P)\right)}{\pi \rho^{2}} \\
\leq(1+\varepsilon) \frac{\mathcal{H}^{2}\left(\Sigma_{0} \cap B_{(1+\delta) \rho}\left(P_{0}\right)\right)_{P_{0}}+\mathcal{H}^{2}\left(\left(\Sigma_{0} \cap B_{(1+\delta) \rho}\left(P_{0}\right)\right)_{P_{0}} \cap \tilde{B}_{(1+\delta) \rho}\left(P_{0}\right)\right)}{\pi((1+\delta) \rho)^{2}},
\end{gathered}
$$

where

$$
\breve{a}=\max \left\{a,-\frac{\delta \rho}{\Lambda}\right\}, \quad \breve{b}=\min \left\{b, \frac{\delta \rho}{\Lambda}\right\}, \quad P_{0}=X_{0} \circ X_{t}^{-1}(P) .
$$

There is one drawback of Corollary 3.3 , which is that the time-span $[\breve{a}, \breve{b}]$ depends on the radius $\rho$ and degenerates as $\rho \searrow 0$. To fix the problem, we appeal to the following lemma from [GJ]. Loosely speaking, it says that the modified area ratio of a surface with free boundary is non-decreasing in radius provided that its mean curvature (and also the curvature of the boundary support surface) is bounded. 
Lemma 3.4. (Monotonicity of Area Ratio)

There is a universal constant $C>0$ with the following property. Let $\Sigma$ be a properly embedded $C^{2}$ surface in $U \subset \mathbb{R}^{3}$ which meets $\Gamma=\partial U$ orthogonally. Suppose that

- Either $B_{1}(O) \subset U$, or $O \in \Gamma$ and $\Gamma$ satisfies the $\kappa$-graph condition for some $0<\kappa \leq 1$;

- There is $\Lambda \geq 0$ so that $\left\|H_{\Sigma}\right\|_{L^{\infty}\left(B_{1}(O)\right)} \leq \Lambda$.

Then for any $P \in \Sigma \cap B_{1}(O)$, the function

$$
r \mapsto e^{C(\Lambda+\kappa) r}\left(\frac{\mathcal{H}^{2}\left(\Sigma \cap B_{r}(P)\right)_{P}+\mathcal{H}^{2}\left(\left(\Sigma \cap B_{r}(P)\right)_{P} \cap \tilde{B}_{r}(P)\right)}{\pi r^{2}}\right)
$$

is non-decreasing for $0<r \leq 1-|P|$.

Combining Lemma 3.1. Corollary 3.3 and Lemma 3.4 we then get the following area ratio estimates for MCF with free boundary and uniformly bounded mean curvature.

Proposition 3.5. (Modified Area Ratio Staying Close to One)

Given $\varepsilon>0$, there exists $\delta>0$ with the following property. Let $\left\{\Sigma_{t}\right\}_{a \leq t \leq b}$ be a properly embedded $C^{2} M C F$ in $U \subset \mathbb{R}^{3}$ with free boundary on $\Gamma=\partial U$, where $a \leq 0 \leq b$ are constants. Suppose that

- Either $B_{1}(O) \subset U$, or $O \in \Gamma$ and $\Gamma$ satisfies the $\kappa$-graph condition for some $0<\kappa \leq 1$;

- There is $K \geq 1$ so that $\left(\Sigma_{0} \cap B_{\frac{\delta}{K}}(P)\right)_{P}$ is a $\delta$-Lipschitz graph for any $P \in \Sigma_{0} \cap B_{\frac{1}{2}}(O)$ (which holds, for instance, when $\left.\left\|A_{\Sigma_{0}}\right\|_{L^{\infty}\left(B_{1}(O)\right)} \leq K\right)$;

- There is $0<\Lambda \leq 1$ so that $\sup _{a \leq t \leq b}\left\|H_{\Sigma_{t}}\right\|_{L^{\infty}\left(B_{1}(O)\right)} \leq \Lambda$.

Then for any $t \in[\breve{a}, \breve{b}], P \in \Sigma_{t} \cap B_{\delta}(O)$, there holds

$$
\sup _{0<r \leq \frac{\delta}{K}} \frac{\mathcal{H}^{2}\left(\Sigma_{t} \cap B_{r}(P)\right)_{P}+\mathcal{H}^{2}\left(\left(\Sigma_{t} \cap B_{r}(P)\right)_{P} \cap \tilde{B}_{r}(P)\right)}{\pi r^{2}} \leq 1+\varepsilon,
$$

where $\breve{a}=\max \left\{a,-\frac{\delta}{\Lambda K}\right\}, \breve{b}=\min \left\{b, \frac{\delta}{\Lambda K}\right\}$.

Next, let's begin the second part of the section with the following lemma. It says that given a complete minimal surface $\Sigma$ in $\mathbb{R}_{+}^{3}$ with free boundary and bounded second fundamental form, if the modified area ratio is sufficiently close to one, it must be a half-plane (cf. [LW]).

Lemma 3.6. There is a universal constant $\vartheta>0$ with the following property. Let $\Sigma$ be a properly embedded $C^{2}$ minimal surface satisfying $\left\|A_{\Sigma}\right\|_{L^{\infty}} \leq K$ for some $K>0$. Suppose that

- Either $\Sigma$ is complete surface in $\mathbb{R}^{3}$ without boundary and

$$
\sup _{r>0} \frac{\mathcal{H}^{2}\left(\Sigma \cap B_{r}(P)\right)_{P}}{\pi r^{2}} \leq 1+\vartheta
$$

for any $P \in \Sigma$; 
- Or $\Sigma$ is a complete surface in $\mathbb{R}_{+}^{3}$ with free boundary on $\partial \mathbb{R}_{+}^{3} \simeq \mathbb{R}^{2}$ and

$$
\sup _{r>0} \frac{\mathcal{H}^{2}\left(\Sigma \cap B_{r}(P)\right)_{P}+\mathcal{H}^{2}\left(\left(\Sigma \cap B_{r}(P)\right)_{P} \cap \tilde{B}_{r}(P)\right)}{\pi r^{2}} \leq 1+\vartheta
$$

for any $P \in \Sigma$.

Then $\Sigma$ is flat, i.e. $A_{\Sigma} \equiv 0$.

Proof. It suffices to prove the first case (which is a lemma in [LW]), since the second case can be reduced to the first case by the method of reflection (see Remark 2.2. (2.7) and Lemma 2.10). Below we sketch the argument in [LW] for the convenience of the reader.

By rescaling, we may assume that $K=1$. For the sake of contradiction, let's suppose that there is a sequence of non-flat, complete, properly embedded $C^{2}$ minimal surfaces $\left\{\Sigma_{i}\right\}_{i \in \mathbb{N}}$ satisfying $0<\left\|A_{\Sigma_{i}}\right\|_{L^{\infty}} \leq 1$ and

$$
\sup _{P \in \Sigma_{i}, r>0} \frac{\mathcal{H}^{2}\left(\Sigma_{i} \cap B_{r}(P)\right)_{P}}{\pi r^{2}} \leq 1+\frac{1}{i}
$$

for all $i$. For each $i \in \mathbb{N}$, choose $P_{i} \in \Sigma_{i}$ so that

$$
2\left|A_{\Sigma_{i}}\left(P_{i}\right)\right| \geq\left\|A_{\Sigma_{i}}\right\|_{L^{\infty}}:=A_{i}>0 .
$$

Let $\hat{\Sigma}_{i}=A_{i}\left(\Sigma_{i}-P_{i}\right)$, then $\hat{\Sigma}_{i}$ is a properly embedded $C^{2}$ minimal surface satisfy$\operatorname{ing}\left\|A_{\hat{\Sigma}_{i}}\right\|_{L^{\infty}} \leq 1,\left|A_{\hat{\Sigma}_{i}}(O)\right| \geq \frac{1}{2}$ and

$$
\sup _{r>0} \frac{\mathcal{H}^{2}\left(\hat{\Sigma}_{i} \cap B_{r}(O)\right)_{O}}{\pi r^{2}} \leq 1+\frac{1}{i} .
$$

By the compactness theorem for the space of minimal surfaces, it follows that a subsequence of $\left\{\hat{\Sigma}_{i}\right\}_{i \in \mathbb{N}}$ converges in the $C^{2}$ topology to a complete minimal surface $\hat{\Sigma}$, which satisfies $\left\|A_{\hat{\Sigma}_{i}}\right\|_{L^{\infty}}=1,\left|A_{\hat{\Sigma}}(O)\right| \geq \frac{1}{2}$ and

$$
\sup _{r>0} \frac{\mathcal{H}^{2}\left(\hat{\Sigma} \cap B_{r}(O)\right)_{O}}{\pi r^{2}} \leq 1
$$

By the monotonicity formula of minimal surfaces (cf. [A] $)$, it follows that $\left(\hat{\Sigma} \cap B_{1}(O)\right)_{O}$ must be a $C^{2}$ minimal cone (and hence a plane), which contradicts with $\left|A_{\hat{\Sigma}}(O)\right| \geq$ $\frac{1}{2}$.

Now we are in a position to establish Li-Wang's pseudolocality theorem for MCF (cf. [LW]) in the free boundary setting. The proof is based on a rescaling argument, with the help of Proposition 3.5 and Lemma 3.6. To simplify the notation in the proof, let's denote the interior norm for the second fundamental form of MCF by

$$
\llbracket A_{\left\{\Sigma_{t}\right\}} \rrbracket_{B_{R}(O) \times B_{\rho}(0)}
$$$$
=\sup \left\{r\left|A_{\Sigma_{t_{0}}}(P)\right|: t_{0} \in B_{\rho}(0), P \in \Sigma_{t_{0}}, B_{r}(P) \times B_{r^{2}}\left(t_{0}\right) \subset B_{R}(O) \times B_{\rho}(0)\right\} .
$$ 
Proposition 3.7. (Li-Wang's Curvature Estimate for MCF)

There exist $\delta>0$ and $C>0$ with the following property. Let $\left\{\Sigma_{t}\right\}_{-1<t<1}$ be a properly embedded $C^{2} M C F$ in $U \subset \mathbb{R}^{3}$ with free boundary on $\Gamma=\partial U$. Suppose that

- Either $B_{1}(O) \subset U$, or $O \in \Gamma$ and $\Gamma$ is mean convex and satisfies the $\kappa$-graph condition for some $0<\kappa \leq 1$;

- There is $K \geq 1$ so that $\left(\Sigma_{0} \cap B_{\frac{\delta}{K}}(P)\right)_{P}$ is a $\delta$-Lipschitz graph for any $P \in \Sigma_{0} \cap B_{\frac{1}{2}}(O)$ (which holds, for instance, when $\left.\left\|A_{\Sigma_{0}}\right\|_{L^{\infty}\left(B_{1}(O)\right)} \leq K\right)$;

- There is $0<\Lambda \leq 1$ so that $\sup _{|t| \leq 1}\left\|H_{\Sigma_{t}}\right\|_{L^{\infty}\left(B_{1}(O)\right)} \leq \Lambda$.

Then we have

$$
\llbracket A_{\left\{\Sigma_{t}\right\}} \rrbracket_{\left.B_{\frac{\delta}{K}}(O) \times B_{\min \left\{1, \frac{\delta}{\Lambda K}\right.}\right\}^{(0)}} \leq C
$$

in particular, there holds

$$
\sup _{|t| \leq \min \left\{\frac{1}{4}, \frac{\delta}{4 \Lambda K}\right\}}\left\|A_{\Sigma_{t}}\right\|_{L^{\infty}\left(B_{\frac{\delta}{2 K}}(O)\right)} \leq \frac{2 C}{\delta} K .
$$

Proof. Let $\vartheta>0$ be the constant in Lemma 3.6 and $\delta>0$ be the constant in Proposition 3.5 with the choice $\varepsilon=\vartheta$.

Suppose that the proposition does not hold. Then for each $i \in \mathbb{N}$, we can find a MCF $\left\{\Sigma_{t}^{i}\right\}_{-1 \leq t \leq 1}$ in $U_{i}$ with free boundary on $\Gamma_{i}=\partial U_{i}$ so that it satisfies the hypotheses and

$$
C_{i}:=\llbracket A_{\left\{\Sigma_{t}^{i}\right\}} \rrbracket_{B_{\frac{\delta}{K}}(O) \times B_{\min \left\{1, \frac{\delta}{K K}\right\}}(0)} \rightarrow \infty .
$$

For each $i \in \mathbb{N}$, choose $\left(P_{i}, t_{i}, r_{i}\right)$ so that $r_{i}\left|A_{\Sigma_{t}^{i}}\left(P_{i}\right)\right| \geq \frac{C_{i}}{2}$. Let $A_{i}=\left|A_{\Sigma_{t}^{i}}\left(P_{i}\right)\right|$, then we have $A_{i} \geq r_{i} A_{i} \rightarrow \infty$ as $i \rightarrow \infty$. Moreover, by (3.1), we have

$$
\sup _{\left|t-t_{i}\right|<\left(\frac{r_{i}}{2}\right)^{2}} \frac{r_{i}}{2}\left\|A_{\Sigma_{t}}\right\|_{L^{\infty}\left(B_{\frac{r_{i}}{2}}\left(P_{i}\right)\right.} \leq C_{i} \leq 2 r_{i} A_{i},
$$

which implies

$$
\sup _{\left|t-t_{i}\right|<\frac{r_{i}^{2}}{4}}\left\|A_{\Sigma_{t}}\right\|_{L^{\infty}\left(B \frac{r_{i}}{2}\left(P_{i}\right)\right.} \leq 4 A_{i}
$$

Here we have two cases to consider:

- Case 1: $\limsup _{i \rightarrow \infty} A_{i} d_{\Gamma_{i}}\left(P_{i}\right)=\infty$;

- Case 2: $\limsup _{i \rightarrow \infty} A_{i} d_{\Gamma_{i}}\left(P_{i}\right)<R$ for some $R>0$.

Case $1\left(\limsup _{i \rightarrow \infty} A_{i} d_{\Gamma_{i}}\left(P_{i}\right)=\infty\right)$ :

By passing to a subsequence, we may assume that $\lim _{i \rightarrow \infty} A_{i} d_{\Gamma_{i}}\left(P_{i}\right)=\infty$. Let

$$
\hat{\Sigma}_{\tau}^{i}=\left(A_{i}\left(\Sigma_{t_{i}+\frac{\tau}{A_{i}^{2}}}^{i}-P_{i}\right) \cap B_{\frac{1}{2} r_{i} A_{i}}(O)\right)_{O_{\tau}}, \quad|\tau| \leq \frac{1}{4}\left(r_{i} A_{i}\right)^{2},
$$

where $O_{\tau}$ is the "normal trajectory" of $O$ along the flow at time $\tau$. Then we have

$$
\begin{gathered}
\left\|A_{\hat{\Sigma}_{\tau}^{i}}\right\|_{L^{\infty}} \leq 4, \quad\left|A_{\hat{\Sigma}_{0}^{i}}(O)\right|=1, \\
\left\|H_{\hat{\Sigma}_{\tau}^{i}}\right\|_{L^{\infty}} \leq \frac{\Lambda}{A_{i}} \rightarrow 0,
\end{gathered}
$$




$$
\sup _{|\tau|<\frac{1}{4}\left(r_{i} A_{i}\right)^{2}} \sup _{Q \in \hat{\Sigma}_{\tau}^{i}} \sup _{0<r<\operatorname{dist}\left(Q, \partial \hat{\Sigma}_{\tau}^{i}\right)} \frac{\mathcal{H}^{2}\left(\hat{\Sigma}_{\tau}^{i} \cap B_{r}(Q)\right)_{Q}}{\pi r^{2}} \leq 1+\vartheta,
$$

in which the last inequality comes from Proposition 3.5. It follows, by Proposition 2.15, that a subsequence of the rescaled flows converges to a complete, properly embedded minimal surface $\hat{\Sigma}$, which satisfies

$$
\begin{aligned}
\left\|A_{\hat{\Sigma}}\right\|_{L^{\infty}} \leq 4, \quad\left|A_{\hat{\Sigma}}(O)\right| & =1, \\
\sup _{Q \in \hat{\Sigma}} \sup _{r>0} \frac{\mathcal{H}^{2}\left(\hat{\Sigma} \cap B_{r}(Q)\right)_{Q}}{\pi r^{2}} & \leq 1+\vartheta .
\end{aligned}
$$

This contradicts Lemma 3.6

Case $2\left(\lim \sup _{i \rightarrow \infty} A_{i} d_{\Gamma_{i}}\left(P_{i}\right)<R\right.$ for some $\left.R>0\right)$ :

Let's first choose $\stackrel{\circ}{P}_{i} \in \Gamma_{i}$ so that $\left|\stackrel{\circ}{P}_{i}-P_{i}\right|=d_{\Gamma_{i}}\left(P_{i}\right)$. For for $i \gg 1$ (so that $r_{i} A_{i}>R$ ), let

$$
\begin{gathered}
\mathcal{P}_{i}=A_{i}\left(P_{i}-\stackrel{\circ}{P}_{i}\right) \in B_{R}(O) ; \\
\hat{\Sigma}_{\tau}^{i}=\left(A_{i}\left(\Sigma_{t_{i}+\frac{\tau}{A_{i}^{2}}}^{i}-\stackrel{\circ}{P}_{i}\right) \cap B_{\frac{1}{2} r_{i} A_{i}}\left(\mathcal{P}_{i}\right)\right)_{\mathcal{P}_{i}(\tau)}, \quad|\tau| \leq \frac{1}{4}\left(r_{i} A_{i}\right)^{2} ; \\
\hat{U}_{i}=A_{i}\left(U_{i}-\stackrel{\circ}{P}_{i}\right), \quad \partial \hat{U}_{i}=\hat{\Gamma}_{i}=A_{i}\left(\Gamma_{i}-\stackrel{\circ}{P}_{i}\right) .
\end{gathered}
$$

where $\mathcal{P}_{i}(\tau)$ is the "normal trajectory" of $\mathcal{P}_{i}$ along the flow at time $\tau$. Then $\hat{\Gamma}_{i}$ satisfies $\kappa_{i}$-graph condition, where $\kappa_{i}=\kappa A_{i}^{-1} \rightarrow 0$, and

$$
\begin{gathered}
\left\|A_{\hat{\Sigma}_{\tau}^{i}}\right\|_{L^{\infty}} \leq 4, \quad\left|A_{\hat{\Sigma}_{0}^{i}}\left(\mathcal{P}_{i}\right)\right|=1, \\
\left\|H_{\hat{\Sigma}_{\tau}^{i}}\right\|_{L^{\infty}} \leq \frac{\Lambda}{A_{i}} \rightarrow 0,
\end{gathered}
$$

and

$$
\frac{\mathcal{H}^{2}\left(\hat{\Sigma}_{\tau}^{i} \cap B_{r}(Q)\right)_{Q}+\mathcal{H}^{2}\left(\left(\hat{\Sigma}_{\tau}^{i} \cap B_{r}(Q)\right)_{Q} \cap \tilde{B}_{r}(Q)\right)}{\pi r^{2}} \leq 1+\vartheta
$$

for all $(Q, \tau, r)$ satisfying

$$
|\tau|<\frac{1}{4}\left(r_{i} A_{i}\right)^{2}, \quad Q \in \hat{\Sigma}_{\tau}^{i}, \quad B_{r}(Q) \subset B_{\frac{1}{2} r_{i} A_{i}}\left(\mathcal{P}_{i}\right) .
$$

Note that the last inequality comes from Proposition 3.5. Passing to a subsequence, we may assume $\mathcal{P}_{i} \rightarrow \mathcal{P} \in B_{R}(O)$ and (by Proposition 2.15) that the rescaled flows converge to a complete, properly embedded minimal surface $\hat{\Sigma}$ in $\mathbb{R}_{+}^{3}$ with free boundary on $\partial \mathbb{R}_{+}^{3} \simeq \mathbb{R}^{2}$. The limiting minimal surface satisfies

$$
\begin{gathered}
\left\|A_{\hat{\Sigma}}\right\|_{L^{\infty}} \leq 4, \quad\left|A_{\hat{\Sigma}}(\mathcal{P})\right|=1, \\
\sup _{Q \in \hat{\Sigma}} \sup _{r>0} \frac{\mathcal{H}^{2}\left(\hat{\Sigma} \cap B_{r}(Q)\right)_{Q}+\mathcal{H}^{2}\left(\left(\hat{\Sigma} \cap B_{r}(Q)\right)_{Q} \cap \tilde{B}_{r}(Q)\right)}{\pi r^{2}} \leq 1+\vartheta,
\end{gathered}
$$

which contradicts Lemma 3.6 . 
To apply Proposition 3.7 one of the conditions to be satisfied is that we need to know in what scale can we write the surface as a local graph with small gradient. Sometimes this is not known in advance, especially when proving our main theorem. Instead, we would like to replace this condition by the smallness of $L^{2}$ norm of the second fundamental form, which is what we called the small energy theorem. The key to making the transition is through the following lemma and its corollary (see Corollary 3.9).

Lemma 3.8. (High-Curvature and Energy Concentration)

Given $K \geq 5$, there exists $\epsilon>0$ with the following property. Let $\left\{\Sigma_{t}\right\}_{-1 \leq t \leq 1}$ be a properly embedded $C^{2} M C F$ in $U \subset \mathbb{R}^{3}$ with free boundary on $\Gamma=\partial U$. Suppose that

- Either $B_{1}(O) \subset U$, or $O \in \Gamma$ and $\Gamma$ is mean convex and satisfies the $\kappa$-graph condition for some $0<\kappa \leq 1$;

- There is $0<\Lambda \leq 1$ so that $\sup _{|t| \leq 1}\left\|H_{\Sigma_{t}}\right\|_{L^{\infty}\left(B_{1}(O)\right)} \leq \Lambda$;

- There holds $\sup \left\{r\left|A_{\Sigma_{0}}(P)\right|: P \in \Sigma_{0}, B_{r}(P) \subset B_{1}(O)\right\}>K$.

Then we have

$$
\int_{\Sigma_{0} \cap B_{1}(O)}\left|A_{\Sigma_{0}}\right|^{2} d \mathcal{H}^{2}>\epsilon
$$

Proof. Suppose the contrary. Then for each $i \in \mathbb{N}$, there is a MCF $\left\{\Sigma_{t}^{i}\right\}_{-1 \leq t \leq 1}$ in $U_{i}$ with free boundary on $\Gamma_{i}=\partial U_{i}$, which satisfies the hypotheses and

$$
\int_{\Sigma_{0}^{i} \cap B_{1}(O)}\left|A_{\Sigma_{0}^{i}}\right|^{2} d \mathcal{H}^{2} \leq \frac{1}{i} .
$$

For each $i \in \mathbb{N}$, choose $P_{i} \in \Sigma_{0}^{i}$ and $0<r_{i}<1$ so that

$$
r_{i}\left|A_{\Sigma_{0}^{i}}\left(P_{i}\right)\right| \geq \frac{1}{2} \sup \left\{r\left|A_{\Sigma_{0}^{i}}(P)\right|: P \in \Sigma_{0}^{i}, B_{r}(P) \subset B_{1}(O)\right\}>\frac{K}{2} .
$$

Note that $\left\|A_{\Sigma_{0}^{i}}\right\|_{L^{\infty}\left(B_{\frac{1}{2} r_{i}}\left(P_{i}\right)\right)} \leq 4 A_{i}$, where $A_{i}=\left|A_{\Sigma_{0}^{i}}\left(P_{i}\right)\right|$.

Let $0<\alpha \leq \frac{1}{4}$ be a small number to be determined. By passing to a subsequence, we may assume that

- Either $\lim \sup _{i \rightarrow \infty} A_{i} d_{\Gamma_{i}}\left(P_{i}\right)<\alpha($ Case 1);

- Or $\lim \sup _{i \rightarrow \infty} A_{i} d_{\Gamma_{i}}\left(P_{i}\right) \geq \alpha$ (Case 2).

Case $1\left(\limsup _{i \rightarrow \infty} A_{i} d_{\Gamma_{i}}\left(P_{i}\right)<\alpha\right)$ :

Choose $\stackrel{\circ}{P}_{i} \in \Gamma_{i}$ so that $\left|\stackrel{\circ}{P}_{i}-P_{i}\right|=d_{\Gamma_{i}}\left(P_{i}\right)$. Define

$$
\mathcal{P}_{i}=A_{i}\left(P_{i}-\stackrel{\circ}{P}_{i}\right), \quad \hat{\Sigma}_{\tau}^{i}=A_{i}\left(\Sigma_{\tau A_{i}^{-2}}^{i}-\stackrel{\circ}{P}_{i}\right), \quad \hat{U}_{i}=A_{i}\left(U_{i}-\stackrel{\circ}{P}_{i}\right) .
$$

Then $\hat{\Gamma}_{i}:=\partial \hat{U}_{i}=A_{i}\left(\Gamma_{i}-\stackrel{\circ}{P}_{i}\right)$ satisfies $\kappa_{i}$-graph condition, where $\kappa_{i}=\kappa A_{i}^{-1} \leq 1$, and

$$
\begin{gathered}
\left\|A_{\hat{\Sigma}_{0}^{i}}\right\|_{L^{\infty}\left(B_{\frac{1}{2} r_{i} A_{i}}\left(\mathcal{P}_{i}\right)\right.} \leq 4, \quad\left|A_{\hat{\Sigma}_{0}^{i}}\left(\mathcal{P}_{i}\right)\right|=1, \\
\sup _{|\tau| \leq A_{i}^{2}}\left\|H_{\hat{\Sigma}_{\tau}^{i}}\right\|_{L^{\infty}\left(B_{\frac{1}{2} r_{i} A_{i}}\left(\mathcal{P}_{i}\right)\right)} \leq \frac{\Lambda}{A_{i}} \leq 1 \\
\int_{\hat{\Sigma}_{0}^{i} \cap B_{\frac{1}{2} r_{i} A_{i}}\left(\mathcal{P}_{i}\right)}\left|A_{\hat{\Sigma}_{0}^{i}}\right|^{2} d \mathcal{H}^{2} \leq \frac{1}{i}
\end{gathered}
$$


Note that $B_{\frac{1}{2} r_{i} A_{i}}\left(\mathcal{P}_{i}\right) \supset B_{1}(O)$ since $\frac{1}{2} r_{i} A_{i} \geq \frac{K}{4}>\frac{5}{4}$ and $\mathcal{P}_{i} \in B_{\alpha}(O)$. By Proposition 3.7, there exist universal constants $\delta>0$ and $C>0$ so that

$$
\sup _{|\tau| \leq \delta^{2}}\left\|A_{\hat{\Sigma}_{\tau}^{i}}\right\|_{L^{\infty}\left(B_{\delta}(O)\right)} \leq C .
$$

It follows, by Proposition 2.15 and passing to a subsequence, that $\mathcal{P}_{i} \rightarrow \mathcal{P} \in B_{2 \alpha}(O)$ and $\left\{\hat{\Sigma}_{\tau}^{i}\right\} \rightarrow\left\{\hat{\Sigma}_{\tau}\right\}$. The limiting $\operatorname{MCF}\left\{\hat{\Sigma}_{\tau}\right\}$ satisfies

$$
\int_{\hat{\Sigma}_{0} \cap B_{\delta}(O)}\left|A_{\hat{\Sigma}_{0}}\right|^{2} d \mathcal{H}^{2}=0, \quad\left|A_{\hat{\Sigma}_{0}}(\mathcal{P})\right|=1,
$$

which is a contradiction if we choose $\alpha<\frac{\delta}{2}$.

Case $2\left(\limsup _{i \rightarrow \infty} A_{i} d_{\Gamma_{i}}\left(P_{i}\right) \geq \alpha\right)$ :

Let

$$
\hat{\Sigma}_{\tau}^{i}=\frac{A_{i}}{\alpha}\left(\Sigma_{\tau\left(\frac{\alpha}{A_{i}}\right)^{2}}-P_{i}\right) .
$$

Then we have

$$
\begin{gathered}
\left\|A_{\hat{\Sigma}_{0}^{i}}\right\|_{L^{\infty}\left(B_{\frac{1}{2} r_{i} \frac{A_{i}}{\alpha}}(O)\right)} \leq 4 \alpha, \quad\left|A_{\hat{\Sigma}_{0}^{i}}(O)\right|=\alpha, \\
\left.\sup _{|\tau| \leq\left(\frac{A_{i}}{\alpha}\right)^{2}}\left\|H_{\hat{\Sigma}_{\tau}^{i} \|^{\infty}}\right\|\right|_{L^{\infty}\left(B_{\frac{1}{2} r_{i} \frac{A_{i}}{\alpha}}(O)\right)} \leq \frac{\alpha \Lambda}{A_{i}} \leq 1, \\
\int_{\hat{\Sigma}_{0}^{i} \cap B_{\frac{1}{2} r_{i} \frac{A_{i}}{\alpha}}(O)}\left|A_{\hat{\Sigma}_{0}^{i}}\right|^{2} d \mathcal{H}^{2} \leq \frac{1}{i} .
\end{gathered}
$$

Proposition 3.7 then implies that there exist $\delta>0$ and $C>0$ so that

$$
\sup _{|\tau| \leq \delta^{2}}\left\|A_{\hat{\Sigma}_{\tau}^{i}}\right\|_{L^{\infty}\left(B_{\delta}(O)\right)} \leq C .
$$

It follows, by Proposition 2.15, that a subsequence of the flows converges to a limiting $\operatorname{MCF}\left\{\hat{\Sigma}_{\tau}\right\}$, which satisfies

$$
\int_{\hat{\Sigma}_{0} \cap B_{\delta}(O)}\left|A_{\hat{\Sigma}_{0}}\right|^{2} d \mathcal{H}^{2}=0, \quad\left|A_{\hat{\Sigma}_{0}}(O)\right|=\alpha,
$$

so we get a contradiction.

Setting $K=5$ in Lemma 3.8 we then get the following corollary.

Corollary 3.9. There exists $\epsilon>0$ with the following property. Let $\left\{\Sigma_{t}\right\}_{-1 \leq t \leq 1}$ be a properly embedded $C^{2} M C F$ in $U \subset \mathbb{R}^{3}$ with free boundary on $\Gamma=\partial U$. Suppose that

- Either $B_{1}(O) \subset U$, or $O \in \Gamma$ and $\Gamma$ is mean convex and satisfies the $\kappa$-graph condition for some $\kappa \leq 1$

- There is $0<\Lambda \leq 1$ so that $\sup _{|t| \leq 1}\left\|H_{\Sigma_{t}}\right\|_{L^{\infty}\left(B_{1}(O)\right)} \leq \Lambda$;

- There holds $\int_{\Sigma_{0} \cap B_{1}(O)}\left|A_{\Sigma_{0}}\right|^{2} d \mathcal{H}^{2} \leq \epsilon$. 
Then we have

$$
\sup \left\{r\left|A_{\Sigma_{0}}(P)\right|: P \in \Sigma_{0}, B_{r}(P) \subset B_{1}(O)\right\} \leq 5,
$$

which, in particular, implies $\left\|A_{\Sigma_{0}}\right\|_{L^{\infty}\left(B_{\frac{1}{2}}(O)\right)} \leq 10$.

Thanks to Corollary 3.9, Proposition 3.7 can be improved as follows (cf. [LW]).

Proposition 3.10. (Li-Wang's Small Energy Theorem)

There exist $\epsilon>0$ and $C>0$ with the following property. Let $\left\{\Sigma_{t}\right\}_{-1<t<1}$ be a properly embedded $C^{2} M C F$ in $U \subset \mathbb{R}^{3}$ with free boundary on $\Gamma=\partial U$. Suppose that

- Either $B_{1}(O) \subset U$, or $O \in \Gamma$ and $\Gamma$ is mean convex and satisfies the $\kappa$-graph condition for some $0<\kappa \leq 1$;

- There holds $\int_{\Sigma_{0} \cap B_{1}(O)}\left|A_{\Sigma_{0}}\right|^{2} d \mathcal{H}^{2} \leq \epsilon$;

- There is $0<\Lambda \leq 1$ so that $\sup _{|t| \leq 1}\left\|H_{\Sigma_{t}}\right\|_{L^{\infty}\left(B_{1}(O)\right)} \leq \Lambda$.

Then we have

$$
\sup _{|t| \leq \min \left\{\frac{1}{4}, \frac{\epsilon}{4 \Lambda}\right\}}\left\|A_{\Sigma_{t}}\right\|_{L^{\infty}\left(B_{\frac{\epsilon}{2}}(O)\right)} \leq C
$$

\section{HYPOTHESES}

In this section we will specify the hypotheses of our main theorem. From now on, let $\left\{\boldsymbol{\Sigma}_{t}\right\}_{0 \leq t<T}$ be a compact, embedded $C^{2}$ MCF in $\boldsymbol{U} \subset \mathbb{R}^{3}$ with free boundary on $\boldsymbol{\Gamma}=\partial \boldsymbol{U}$, where $T>0$ is a finite constant. We assume that

- $\boldsymbol{\Gamma}=\partial \boldsymbol{U}$ is a properly embedded $C^{3,1}$ surface which satisfies the $\boldsymbol{\kappa}$-graph condition for some $\boldsymbol{\kappa}>0$ (see Definition 2.3) and is mean convex, i.e.

$$
H_{\boldsymbol{\Gamma}}=-\nabla_{\boldsymbol{\Gamma}} \cdot \boldsymbol{\nu} \geq 0
$$

where $\boldsymbol{\nu}$ is the inward, unit normal vector of $\boldsymbol{\Gamma}$;

- The mean curvature of $\left\{\boldsymbol{\Sigma}_{t}\right\}_{0 \leq t<T}$ is uniformly bounded, i.e.

$$
\sup _{0 \leq t<T}\left\|H_{\boldsymbol{\Sigma}_{t}}\right\|_{L^{\infty}} \leq \boldsymbol{\Lambda}<\infty
$$

- The perimeter of $\left\{\boldsymbol{\Sigma}_{t}\right\}_{0 \leq t<T}$ is uniformly bounded, i.e.

$$
\sup _{0 \leq t<T} \mathcal{H}^{1}\left(\partial \boldsymbol{\Sigma}_{t}\right) \leq \boldsymbol{l}<\infty .
$$

Note that in order to distinguish from the generic MCF that appeared in the previous sections, we use boldface to denote the specific MCF in the main theorem. Also, in the proof we only need $\boldsymbol{\Gamma}$ to satisfy the $\boldsymbol{\kappa}$-graph condition in the region where the flow exists (i.e. the support of the flow). Actually, the support of the flow is bounded as $\boldsymbol{\Sigma}_{0}$ is compact and its mean curvature stays uniformly bound. Since every properly embedded $C^{3,1}$ surface locally satisfies the $\boldsymbol{\kappa}$-graph condition (with $\boldsymbol{\kappa}$ depending on the given bounded region), one can regard this condition as a byproduct of the other conditions.

The goal of this paper is to show that the second fundamental form of $\left\{\boldsymbol{\Sigma}_{t}\right\}_{0<t<T}$ is uniformly bounded; whence, by $[\mathbf{S}]$ the flow can be extended (see Theorem [6.1). The proof begins in this section and will be completed in Section 6. For the rest of this section, we will first show that the $L^{2}$ norm of the second fundamental form is uniformly bounded. Then we will use that to prove the condensation compactness theorem for sequences of parabolic rescaling of the flow. 
Lemma 4.1. (Uniformly Bounded Energy)

The $L^{2}$ norm of the second fundamental form is uniformly bounded. More precisely, there holds

$$
\int_{\boldsymbol{\Sigma}_{t}}\left|A_{\boldsymbol{\Sigma}_{t}}\right|^{2} d \mathcal{H}^{2} \leq C\left(\boldsymbol{\Lambda}, \boldsymbol{\kappa}, \boldsymbol{l}, \mathcal{H}^{2}\left(\boldsymbol{\Sigma}_{0}\right), \chi\left(\boldsymbol{\Sigma}_{0}\right)\right)
$$

for $0 \leq t<T$.

Proof. Using the normal parametrization of the flow, i.e.

$$
X_{t}=X(\cdot, t): M^{2} \times[0, T) \rightarrow \boldsymbol{U} \subset \mathbb{R}^{3}, \quad \partial_{t} X_{t}=\vec{H}_{\boldsymbol{\Sigma}_{t}},
$$

we have

$$
\partial_{t} d \boldsymbol{\mu}_{t}=-H_{\boldsymbol{\Sigma}_{t}}^{2} d \boldsymbol{\mu}_{t}
$$

where $d \boldsymbol{\mu}_{t}$ is the pull-back measure of $\boldsymbol{\Sigma}_{t}$ on $M$. It follows that

$$
\sup _{0 \leq t<T} \mathcal{H}^{2}\left(\boldsymbol{\Sigma}_{t}\right) \leq \mathcal{H}^{2}\left(\boldsymbol{\Sigma}_{0}\right)<\infty .
$$

Next, for each $P \in \boldsymbol{U} \cup \boldsymbol{\Gamma}$, let

$$
r_{P}=\left\{\begin{array}{c}
d_{\boldsymbol{\Gamma}}(P), \quad \text { if } \quad P \in \boldsymbol{U} \\
\frac{1}{\sqrt{2}}\left(\frac{3}{320}\right)^{\frac{5}{2}} \boldsymbol{\kappa}^{-1}, \quad \text { if } P \in \boldsymbol{\Gamma}
\end{array} .\right.
$$

Lemma 2.6 yields the following area ratio estimate (cf. [E, $\mathrm{K}]$ ):

$$
\frac{\mathcal{H}^{2}\left(\boldsymbol{\Sigma}_{t} \cap B_{R \sqrt{T-t}}(P)\right)}{R^{2}(T-t)} \leq C(\boldsymbol{\kappa}) \frac{\mathcal{H}^{2}\left(\boldsymbol{\Sigma}_{T-\frac{5}{14} r_{P}^{2}} \cap B_{r_{P}}(P)\right)}{r_{P}^{2}} \leq C\left(\boldsymbol{\kappa}, \mathcal{H}^{2}\left(\boldsymbol{\Sigma}_{0}\right), r_{P}\right)
$$

for $R>1, T-\frac{1}{10}\left(\frac{r_{P}}{R}\right)^{2}<t<T$.

By the Gauss-Bonnet theorem, for each $t \in[0, T)$ we have

$$
\int_{\boldsymbol{\Sigma}_{t}} K_{\boldsymbol{\Sigma}_{t}} d \mathcal{H}^{2}+\int_{\gamma_{t}} \vec{k}_{\gamma_{t}} \cdot \boldsymbol{\nu} d \mathcal{H}^{1}=2 \pi \chi\left(\boldsymbol{\Sigma}_{t}\right)
$$

where $K_{\boldsymbol{\Sigma}_{t}}$ is the Gauss curvature of $\boldsymbol{\Sigma}_{t}$,

$$
\gamma_{t}:=\partial \boldsymbol{\Sigma}_{t}=\boldsymbol{\Sigma}_{t} \cap \boldsymbol{\Gamma}
$$

is the boundary curve, $\vec{k}_{\gamma_{t}}=D_{T_{\gamma_{t}}} T_{\gamma_{t}}$ is the curvature vector of $\gamma_{t}$ in $\mathbb{R}^{3}, T_{\gamma_{t}}$ is the unit tangent vector of $\gamma_{t}$, and $\chi\left(\boldsymbol{\Sigma}_{t}\right)$ is the Euler characteristic of $\boldsymbol{\Sigma}_{t}$. Note that

$$
\begin{gathered}
K_{\boldsymbol{\Sigma}_{t}}=\frac{1}{2}\left(H_{\boldsymbol{\Sigma}_{t}}^{2}-\left|A_{\boldsymbol{\Sigma}_{t}}\right|^{2}\right), \\
\vec{k}_{\gamma_{t}} \cdot \boldsymbol{\nu}=D_{T_{\gamma_{t}}} T_{\gamma_{t}} \cdot \boldsymbol{\nu}=-T_{\gamma_{t}} \cdot D_{T_{\gamma_{t}}} \boldsymbol{\nu}=A_{\boldsymbol{\Gamma}}\left(T_{\gamma_{t}}, T_{\gamma_{t}}\right), \\
\chi\left(\boldsymbol{\Sigma}_{t}\right)=\chi\left(\boldsymbol{\Sigma}_{0}\right) .
\end{gathered}
$$

It follows from (4.2), (4.3) and (4.5) that

$$
\begin{aligned}
\int_{\boldsymbol{\Sigma}_{t}}\left|A_{\boldsymbol{\Sigma}_{t}}\right|^{2} d \mathcal{H}^{2}= & \int_{\boldsymbol{\Sigma}_{t}} H_{\boldsymbol{\Sigma}_{t}}^{2} d \mathcal{H}^{2}+2 \int_{\gamma_{t}} A_{\boldsymbol{\Gamma}}\left(T_{\gamma_{t}}, T_{\gamma_{t}}\right) d \mathcal{H}^{1}-4 \pi \chi\left(\boldsymbol{\Sigma}_{0}\right) \\
& \lesssim \boldsymbol{\Lambda}^{2} \mathcal{H}^{2}\left(\boldsymbol{\Sigma}_{0}\right)+2 \boldsymbol{\kappa} \boldsymbol{l}-4 \pi \chi\left(\boldsymbol{\Sigma}_{0}\right) .
\end{aligned}
$$

The following remark points out that the energy concentrates at only finitely many points; hence there are at most finitely many singularities at time $T$. 
Remark 4.2. Let

$$
\omega_{t}=\left|A_{\boldsymbol{\Sigma}_{t}}\right|^{2}\left\lfloor d \mathcal{H}^{2} .\right.
$$

By (4.4) and compactness, given any sequence $t_{i} \nearrow T$, there is a subsequence (still denoted by $\left\{t_{i}\right\}$ for simplicity of notations) so that $\omega_{t_{i}} \rightarrow \omega$ in the sense of Radon measure in $\mathbb{R}^{3}$. It follows from the regularity of Radon measures that $\omega_{t_{i}}(\mathcal{B}) \rightarrow \omega(\mathcal{B})$ for any bounded Borel set $\mathcal{B}$ satisfying $\omega(\partial \mathcal{B})=0$. As a result (together with the local finiteness of $\omega$ ), we have

$$
\omega_{t_{i}}\left(B_{r}(P)\right) \rightarrow \omega\left(B_{r}(P)\right)
$$

for every $P \in \mathbb{R}^{3}$ and almost every $r>0$. Let's define

$$
\mathcal{S}=\left\{P \in \mathbb{R}^{3} \mid \lim _{i \rightarrow \infty} \omega_{t_{i}}\left(B_{r}(P)\right) \geq \epsilon \quad \forall r>0\right\},
$$

where $\epsilon$ is the constant in Proposition 3.10. Note that

$$
\mathcal{H}^{0}(\mathcal{S}) \leq \frac{1}{\epsilon} \limsup _{t \nearrow T} \int_{\boldsymbol{\Sigma}_{t}}\left|A_{\boldsymbol{\Sigma}_{t}}\right|^{2} d \mathcal{H}^{2} \leq C\left(\boldsymbol{\Lambda}, \boldsymbol{\kappa}, \boldsymbol{l}, \mathcal{H}^{2}\left(\boldsymbol{\Sigma}_{0}\right), \chi\left(\boldsymbol{\Sigma}_{0}\right)\right)
$$

by (4.4). By Proposition 3.10 and Proposition 2.12, one can conclude that for any limit point $P$ of $\left\{\boldsymbol{\Sigma}_{t}\right\}$ (as $t \nearrow T$ ) which is not in $\mathcal{S}$, there exists $r>0$ so that $\left\{\boldsymbol{\Sigma}_{t} \cap B_{r}(P)\right\}$ is $C^{2,1}$ up to time $T$. Therefore, there are at most finitely many singularities of $\left\{\boldsymbol{\Sigma}_{t}\right\}$ as $t \nearrow T$.

Below we consider the parabolic rescaling of $\left\{\boldsymbol{\Sigma}_{t}\right\}$ about a singular point on the boundary and prove the condensation compactness theorem.

Proposition 4.3. (Condensation Compactness)

Given $P \in \mathcal{S} \cap \boldsymbol{\Gamma}$ and a sequence $\left\{\lambda_{i} \searrow 0\right\}_{i \in \mathbb{N}}$. Let

$$
\boldsymbol{\Sigma}_{\tau}^{(P, T), \lambda_{i}}=\frac{1}{\lambda_{i}}\left(\boldsymbol{\Sigma}_{T+\lambda_{i}^{2} \tau}-P\right), \quad-\frac{T}{\lambda_{i}^{2}} \leq \tau<0
$$

and

$$
\boldsymbol{U}^{P, \lambda_{i}}=\frac{1}{\lambda_{i}}(\boldsymbol{U}-P), \quad \boldsymbol{\Gamma}^{P, \lambda_{i}}=\partial \boldsymbol{U}^{P, \lambda_{i}}=\frac{1}{\lambda_{i}}(\boldsymbol{\Gamma}-P) .
$$

Then there exist a half plane $\Pi$ which meets $\lim _{i \rightarrow \infty} \Gamma^{P, \lambda_{i}} \simeq \mathbb{R}^{2}$ orthogonally, a finite set $\mathfrak{S}_{P} \subset \Pi$, and an integer $m \in \mathbb{N}$ so that a subsequence of (4.9) converges to $\{\Pi\}_{-\infty<\tau<0}$ with multiplicity $m$ away from $\mathfrak{S}_{P} \times(-\infty, 0)$. Moreover, we have

$$
\mathcal{H}^{0}\left(\mathfrak{S}_{P}\right) \leq C\left(\boldsymbol{\Lambda}, \boldsymbol{\kappa}, \boldsymbol{l}, \mathcal{H}^{2}\left(\boldsymbol{\Sigma}_{0}\right), \chi\left(\boldsymbol{\Sigma}_{0}\right)\right) .
$$

An analogous result holds for $P \in \mathcal{S} \cap \boldsymbol{U}$, in which case $\Pi$ is a plane.

Proof. Throughout the proof, we will assume that $P \in \mathcal{S} \cap \boldsymbol{\Gamma}$. The result for $P \in \mathcal{S} \cap \boldsymbol{U}$ follows from the same argument.

Firstly, note that $\left\{\boldsymbol{\Sigma}_{\tau}^{(P, T), \lambda_{i}}\right\}$ is a MCF in $\boldsymbol{U}^{P, \lambda_{i}}$ with free boundary on $\boldsymbol{\Gamma}^{P, \lambda_{i}}$, which is mean convex and satisfies $\lambda_{i} \kappa$-graph condition. Also, by (4.6), (4.2) and (4.4), we have

$$
\begin{gathered}
\sup _{R>1} \sup _{-\frac{1}{10}\left(\frac{r_{p}}{\lambda_{i} R}\right)^{2}<\tau<0} \frac{\mathcal{H}^{2}\left(\boldsymbol{\Sigma}_{\tau}^{(P, T), \lambda_{i}} \cap B_{R \sqrt{-\tau}}(O)\right)}{R^{2}(-\tau)} \leq C\left(\boldsymbol{\kappa}, \mathcal{H}^{2}\left(\boldsymbol{\Sigma}_{0}\right), r_{p}\right), \\
\sup _{-\frac{T}{\lambda_{i}^{2}} \leq \tau<0}\left\|H_{\boldsymbol{\Sigma}_{\tau}^{(P, T), \lambda_{i}}}\right\|_{L^{\infty}} \leq \lambda_{i} \boldsymbol{\Lambda},
\end{gathered}
$$




$$
\int_{\boldsymbol{\Sigma}_{-1}^{(P, T), \lambda_{i}}}\left|A_{\boldsymbol{\Sigma}_{-1}^{(P, T), \lambda_{i}}}\right|^{2} d \mathcal{H}^{2} \leq C\left(\boldsymbol{\Lambda}, \boldsymbol{\kappa}, \boldsymbol{l}, \mathcal{H}^{2}\left(\boldsymbol{\Sigma}_{0}\right), \chi\left(\boldsymbol{\Sigma}_{0}\right)\right) .
$$

By a similar argument as in Remark 4.2, the set

$$
\mathfrak{S}_{P}:=\left\{\left.Q \in \mathbb{R}^{3}\left|\lim _{i \rightarrow \infty} \int_{\boldsymbol{\Sigma}_{-1}^{(P, T), \lambda_{i}} \cap B_{r}(Q)}\right| A_{\boldsymbol{\Sigma}_{-1}^{(P, T), \lambda_{i}}}\right|^{2} d \mathcal{H}^{2}\left(B_{r}(Q)\right) \geq \epsilon \quad \forall r>0\right\}
$$

is finite, where $\epsilon$ is the constant in Proposition 3.10, and

$$
\mathcal{H}^{0}\left(\mathfrak{S}_{P}\right) \leq C\left(\boldsymbol{\Lambda}, \boldsymbol{\kappa}, \boldsymbol{l}, \mathcal{H}^{2}\left(\boldsymbol{\Sigma}_{0}\right), \chi\left(\boldsymbol{\Sigma}_{0}\right)\right) .
$$

It follows, by Proposition 3.10, (4.10), (4.11) and Proposition2.15, that $\left\{\boldsymbol{\Sigma}_{\tau}^{(P, T), \lambda_{i}}\right\}$ converges with finite multiplicity to a minimal surface $\{\Pi\}_{-\infty<\tau<0}$ away from $\mathfrak{S}_{P}$. Note that

$$
\boldsymbol{U}^{P, \lambda_{i}} \rightarrow \mathbb{R}_{+}^{3}, \quad \Gamma^{P, \lambda_{i}} \rightarrow \partial \mathbb{R}_{+}^{3} \simeq \mathbb{R}^{2}
$$

and $\Pi$ is orthogonal to $\lim _{i \rightarrow \infty} \Gamma^{P, \lambda_{i}}$. Furthermore, Lemma 2.6 implies that the limiting surface $\Pi$ satisfies the self-shrinker equation

$$
H_{\Pi}+\frac{1}{2} X \cdot N_{\Pi}=0
$$

(cf. [I, B]). Consequently, the minimal surface $\Pi$ must be flat.

\section{Unity of Huisken's Density}

The goal of this section is to show the unity of Huisken's density of $\left\{\boldsymbol{\Sigma}_{t}\right\}_{0<t<T}$ at time $T$. By "unity" we mean that it is one for the interior limit points and one half for the boundary limit points (see Proposition 5.5). We will follow closely the procedure in $[\mathrm{LW}$ to prove that. Our discussion will focus on the boundary limit points since the arguments are similar for the interior limit points.

In order to prove the unity of Huisken's density, we will work with the normalized MCF defined in (5.1). As a consequence of Proposition 4.3, any sequence of timeslices of (5.1) would converge to a half-plane with multiplicity away from (at most) finitely many singularities (see Lemma 5.1]). Following the idea of [LW], we will choose special sequences and use that to prove the unity of Huisken's density by contradiction (see Proposition 5.3, Lemma 5.4 and Proposition 5.5). It then follows from Allard's regularity theorem (cf. [Al]) and Proposition 3.7 that the convergence in Proposition 4.3 is of multiplicity one and without singularities (see Corollary [5.6).

Let's begin our discussion with the parabolic rescaling defined in (4.9). Given $P \in \mathcal{S} \cap \boldsymbol{\Gamma}$ and a sequence $\left\{t_{i} \nearrow T\right\}_{i \in \mathbb{N}}$, let $\lambda_{i}=\sqrt{T-t_{i}}$ and

$$
\begin{gathered}
\boldsymbol{\Sigma}_{\tau}^{(P, T), \lambda_{i}}=\frac{1}{\lambda_{i}}\left(\boldsymbol{\Sigma}_{T+\lambda_{i}^{2} \tau}-P\right) \quad \text { for } \quad-\frac{T}{\lambda_{i}^{2}} \leq \tau<0, \\
\boldsymbol{U}^{P, \lambda_{i}}=\frac{1}{\lambda_{i}}(\boldsymbol{U}-P), \quad \boldsymbol{\Gamma}^{P, \lambda_{i}}=\partial \boldsymbol{U}^{P, \lambda_{i}}=\frac{1}{\lambda_{i}}(\boldsymbol{\Gamma}-P) .
\end{gathered}
$$

Note that $\boldsymbol{\Gamma}^{P, \lambda_{i}}$ satisfies $\lambda_{i} \boldsymbol{\kappa}$-graph condition. As usual, we will parametrize its tubular neighborhood near $O$ by a map $\boldsymbol{\Phi}^{P, \lambda_{i}}$ (defined by Definition 2.4 and Remark (2.5). 
On the other hand, it is very useful to consider the following time-dependent parabolic rescaling of $\left\{\boldsymbol{\Sigma}_{t}\right\}$ (called "normalized MCF"), $\boldsymbol{U}$ and $\boldsymbol{\Gamma}$ :

$$
\begin{gathered}
\Pi_{s}:=\left.\frac{1}{\sqrt{T-t}}\left(\boldsymbol{\Sigma}_{t}-P\right)\right|_{t=T-e^{-s}} \quad \text { for } \quad-\ln T \leq s<\infty, \\
\boldsymbol{U}_{s}:=\left.\frac{1}{\sqrt{T-t}}(\boldsymbol{U}-P)\right|_{t=T-e^{-s}}, \quad \boldsymbol{\Gamma}_{s}:=\partial \boldsymbol{U}_{s}=\left.\frac{1}{\sqrt{T-t}}(\boldsymbol{\Gamma}-P)\right|_{t=T-e^{-s}} .
\end{gathered}
$$

Note that $\Pi_{s}$ has free boundary on $\boldsymbol{\Gamma}_{s}$ and that $\boldsymbol{\Gamma}_{s}$ satisfies $e^{-\frac{s}{2}} \boldsymbol{\kappa}$-graph condition. Likewise, the tubular neighborhood of $\boldsymbol{\Gamma}_{s}$ near $O$ is parametrized by a map $\boldsymbol{\Phi}_{s}$ (defined by Definition 2.4 and Remark 2.5).

As a remark, let $s_{i}=-\ln \left(T-t_{i}\right)$, then one can see that

$$
\begin{gathered}
\Pi_{s_{i}}=\frac{1}{\sqrt{T-t_{i}}}\left(\boldsymbol{\Sigma}_{t_{i}}-P\right)=\boldsymbol{\Sigma}_{-1}^{(P, T), \lambda_{i}}, \\
\boldsymbol{U}_{s_{i}}=\frac{1}{\sqrt{T-t_{i}}}(\boldsymbol{U}-P)=\boldsymbol{U}^{P, \lambda_{i}}, \quad \boldsymbol{\Gamma}_{s_{i}}=\frac{1}{\sqrt{T-t_{i}}}(\boldsymbol{\Gamma}-P)=\boldsymbol{\Gamma}^{P, \lambda_{i}} .
\end{gathered}
$$

More generally, for each $\sigma \in\left[-\ln T-s_{i}, \infty\right)$, we have

$$
\Pi_{s_{i}+\sigma}=\left.\frac{1}{\sqrt{-\tau}} \boldsymbol{\Sigma}_{\tau}^{(P, \boldsymbol{T}), \lambda_{i}}\right|_{\tau=-e^{-\sigma}},
$$

$$
\boldsymbol{U}_{s_{i}+\sigma}=\left.\frac{1}{\sqrt{-\tau}} \boldsymbol{U}^{P, \lambda_{i}}\right|_{\tau=-e^{-\sigma}}, \quad \boldsymbol{\Gamma}_{s_{i}+\sigma}=\partial \boldsymbol{U}_{s_{i}+\sigma}=\left.\frac{1}{\sqrt{-\tau}} \boldsymbol{\Gamma}^{P, \lambda_{i}}\right|_{\tau=-e^{-\sigma}} .
$$

The following lemma is a paraphrasing of Proposition 4.3 in terms of $\left\{\Pi_{s_{i}+\sigma}\right\}$.

Lemma 5.1. Given $P \in \mathcal{S} \cap \boldsymbol{\Gamma}$ and a sequence $\left\{s_{i} \nearrow \infty\right\}_{i \in \mathbb{N}}$, there exist a half plane $\Pi$, a finite set $\mathfrak{S}_{P} \subset \Pi$, and an integer $m \in \mathbb{N}$ so that, after passing to a subsequence, $\left\{\Pi_{s_{i}+\sigma}\right\}_{-\ln T-s_{i}<\sigma<\infty}$ converges to $\{\Pi\}_{-\infty<\sigma<\infty}$ with multiplicity $m$ away from $\underset{-\infty<\sigma<\infty}{\cup} e^{\frac{\sigma}{2} \mathfrak{S}_{P} \times\{\sigma\}}$.

The half plane $\Pi$ meets $\lim _{i \rightarrow \infty} \boldsymbol{\Gamma}_{s_{i+\sigma}} \simeq \mathbb{R}^{2}$ orthogonally. The number of $\mathfrak{S}_{P}$ is bounded by

$$
\mathcal{H}^{0}\left(\mathfrak{S}_{P}\right) \leq C\left(\boldsymbol{\Lambda}, \boldsymbol{\kappa}, \boldsymbol{l}, \mathcal{H}^{2}\left(\boldsymbol{\Sigma}_{0}\right), \chi\left(\boldsymbol{\Sigma}_{0}\right)\right) .
$$

Moreover, Huisken's densitiy of $\left\{\boldsymbol{\Sigma}_{t}\right\}$ at $(P, T)$ (see Lemma 2.7) is given by

$$
\Theta_{\left\{\boldsymbol{\Sigma}_{t}\right\}}(P, T)=\frac{m}{2}
$$

Proof. By Proposition 4.3, there exist a half plane $\Pi$, a finite set $\mathfrak{S}_{P} \subset \Pi$, and an integer $m \in \mathbb{N}$ so that $\left\{\boldsymbol{\Sigma}_{\tau}^{(P, T), \lambda_{i}}\right\} \rightarrow\{\Pi\}$ with multiplicity $m$ away from the set $\mathfrak{S}_{P} \times(-\infty, 0)$. It follows that

$$
\Pi_{s_{i}+\sigma}=\left.\frac{1}{\sqrt{-\tau}} \Sigma_{\tau}^{(P, T), \lambda_{i}}\right|_{\tau=-e^{-\sigma}} \rightarrow \frac{1}{\sqrt{-\tau}} \Pi=\Pi
$$

away from $\underset{-\infty<\sigma<\infty}{\cup} \frac{1}{\sqrt{-\tau}} \mathfrak{S}_{P} \times\{\sigma\}$, where $\tau=-e^{-\sigma}$. Notice that

$$
\left\|H_{\Pi_{s_{i}+\sigma}}\right\|_{L^{\infty}} \leq \boldsymbol{\Lambda} e^{-\frac{1}{2}\left(s_{i}+\sigma\right)} \rightarrow 0 \quad \text { as } \quad i \rightarrow \infty
$$

and $\boldsymbol{\Gamma}_{s_{i}+\sigma}$ satisfies a $\boldsymbol{\kappa} e^{-\frac{1}{2}\left(s_{i}+\sigma\right)}$-graph condition.

On the other hand, let $t_{i}=T-e^{-s_{i}}$, then Lemma 2.7 yields

$$
\Theta_{\left\{\boldsymbol{\Sigma}_{t}\right\}}(P, T)=\lim _{i \rightarrow \infty} \int_{\boldsymbol{\Sigma}_{t_{i}}} e^{85\left(\boldsymbol{\kappa}^{2}\left(T-t_{i}\right)\right)^{\frac{2}{5}}} \eta_{\boldsymbol{\Gamma} ; P, T} \Psi_{\boldsymbol{\Gamma} ; P, T}\left(X, t_{i}\right) d \mathcal{H}^{2}(X)
$$




$$
=\lim _{i \rightarrow \infty} \int_{\Pi_{s_{i}}} e^{85\left(\kappa^{2} e^{-s_{i}}\right)^{\frac{2}{5}}} \eta_{\Gamma_{s_{i}}} \Psi_{\boldsymbol{\Gamma}_{s_{i}}}(Y) d \mathcal{H}^{2}(Y)
$$

where

$$
\begin{gathered}
\eta_{\boldsymbol{\Gamma}_{s_{i}}}(Y)=\left(1-\frac{|Y|^{2}+|\tilde{Y}|^{2}-80}{\left(\frac{1}{2}\left(\boldsymbol{\kappa}^{2} e^{-s_{i}}\right)^{\frac{2}{5}}\left(\boldsymbol{\kappa} e^{-\frac{s_{i}}{2}}\right)^{-1}\right)^{2}}\right)_{+}^{4}, \\
\Psi_{\boldsymbol{\Gamma}_{s_{i}}}(Y)=\frac{1}{4 \pi} \exp \left(-\frac{\frac{1}{2}\left(|Y|^{2}+|\tilde{Y}|^{2}\right)}{4\left(1+16\left(\boldsymbol{\kappa}^{2} e^{-s_{i}}\right)^{\frac{2}{5}}\right)}\right)
\end{gathered}
$$

Due to $\boldsymbol{\Gamma}_{s_{i}} \rightarrow \partial \mathbb{R}^{3} \simeq \mathbb{R}^{2}, \Pi_{s_{i}} \rightarrow \Pi$ with multiplicity $m$ away from $\mathfrak{S}_{P}$, (4.10) and Lemma 3.4 (with $\Sigma$ replaced by $\Pi_{s_{i}}$ ), we get $\Theta_{\left\{\boldsymbol{\Sigma}_{t}\right\}}(P, T)=\frac{m}{2}$.

Next, we would like to choose carefully sequences in Lemma 5.1 in the hope that it could help to prove the unity of Huisken's density. For that purpose, let's make the following definition. Given $\delta>0$, for each $s \in[-\ln T, \infty)$ we define

$$
\boldsymbol{U}_{s}^{\delta \text {-curved }}=\left\{Q \in \boldsymbol{U}_{s} \cap B_{\delta^{-1}}(O) \mid \exists Q^{\prime} \in \Pi_{s} \cap B_{\delta}(Q) \text { s.t. } \delta\left|A_{\Pi_{s}}\left(Q^{\prime}\right)\right| \geq 1\right\} .
$$

Let $\boldsymbol{U}_{s}^{\delta \text {-outer }}$ be the set consisting of all points $Q \in\left(\boldsymbol{U}_{s} \cap B_{\delta^{-1}}(O)\right) \backslash\left(\Pi_{s} \cup \boldsymbol{U}_{s}^{\delta \text {-curved }}\right)$ for which there is a continuous path $\gamma:[0,1) \rightarrow \boldsymbol{U}_{s} \backslash\left(\Pi_{s} \cup \boldsymbol{U}_{s}^{\delta \text {-curved }}\right)$ so that $\gamma(0)=Q$ and $\operatorname{dist}\left(\gamma(\xi), \Pi_{s}\right) \rightarrow \infty$ as $\xi \nearrow 1$. Then we define

$$
\boldsymbol{U}_{s}^{\delta}=\left(\boldsymbol{U}_{s} \cap B_{\delta^{-1}}(O)\right) \backslash\left(\boldsymbol{U}_{s}^{\delta-\text { curved }} \cup \boldsymbol{U}_{s}^{\delta \text {-outer }}\right) .
$$

Loosely speaking, $\boldsymbol{U}_{s}^{\delta}$ is the region in $B_{\delta^{-1}}(O)$ which is enclosed by $\Pi_{s}$ (especially when it has multiple sheets) and away from points of large curvature of $\Pi_{s}$ (cf. $[\mathrm{LW}]$ ).

The next lemma will be used to choose the special sequences in Proposition 5.3

Lemma 5.2. Given $\delta>0$ and $i \in \mathbb{N}$, there exists $s_{i} \geq i$ so that

$$
\sup _{0<\sigma \leq i} \mathcal{H}^{3}\left(\boldsymbol{U}_{s_{i}+\sigma}^{\delta}\right) \leq\left(1+\frac{1}{i}\right) \mathcal{H}^{3}\left(\boldsymbol{U}_{s_{i}}^{\delta}\right) .
$$

Proof. Suppose the contrary, then for every $s \geq i$, there exists $0<\sigma_{s} \leq i$ so that

$$
\mathcal{H}^{3}\left(\boldsymbol{U}_{s+\sigma_{s}}^{\delta}\right)>\left(1+\frac{1}{i}\right) \mathcal{H}^{3}\left(\boldsymbol{U}_{s}^{\delta}\right)
$$

In particular, we have

$$
\mathcal{H}^{3}\left(\boldsymbol{U}_{i+\sigma_{i}}^{\delta}\right)>\left(1+\frac{1}{i}\right) \mathcal{H}^{3}\left(\boldsymbol{U}_{i}^{\delta}\right) \geq 0
$$

Define a sequence $\left\{s_{k}\right\}_{k \in \mathbb{Z}_{+}}$recursively by setting $s_{k+1}=s_{k}+\sigma_{s_{k}}$ for $k \geq 0$ and $s_{0}=i+\sigma_{i}$. Then we have

$$
\mathcal{H}^{3}\left(\boldsymbol{U}_{s_{k}}^{\delta}\right)>\left(1+\frac{1}{i}\right)^{k} \mathcal{H}^{3}\left(\boldsymbol{U}_{s_{0}}^{\delta}\right) \quad \forall k \in \mathbb{N},
$$

which implies $\lim \sup _{k \rightarrow \infty} \mathcal{H}^{3}\left(\boldsymbol{U}_{s_{k}}^{\delta}\right)=\infty$ (a contradiction). 
In the following proposition, we use Lemma 5.2 to choose a special sequence of (5.2). By Lemma 5.1 and Definition 2.4, we can parametrize each flow as a multigraph over a half-plane. In that case, if the multiplicity is not one, the volume of (5.3) can be roughly interpreted as the integral of the difference of the upper and lower graphs, over which we have some uniform control within any finite period of time (see (5.6) ). Moreover, we also derive the equation satisfied by the difference functions.

Proposition 5.3. Given $P \in \mathcal{S} \cap \boldsymbol{\Gamma}$ and $\delta>0$. Suppose that $\Theta_{\left\{\boldsymbol{\Sigma}_{t}\right\}}(P, T)>\frac{1}{2}$, then there exists a sequence $\left\{s_{i} \nearrow \infty\right\}_{i \in \mathbb{N}}$, a half plane $\Pi$, a finite set $\mathfrak{S}_{P} \subset \Pi$ and an integer $m>1$ with the following property. If we assume (without loss of generality) that

$$
\begin{gathered}
\lim _{i \rightarrow \infty} \boldsymbol{U}_{s_{i}}=\left\{\left(x_{1}, x_{2}, x_{3}\right) \mid x_{1}, x_{3} \in \mathbb{R}, x_{2}>0\right\}, \\
\lim _{i \rightarrow \infty} \boldsymbol{\Gamma}_{s_{i}}=\left\{\left(x_{1}, 0, x_{3}\right) \mid x_{1}, x_{3} \in \mathbb{R}\right\}, \\
\Pi=\left\{\left(x_{1}, x_{2}, 0\right) \mid x_{1} \in \mathbb{R}, x_{2} \geq 0\right\},
\end{gathered}
$$

then the rescaled flow $\left\{\Pi_{s_{i}+\sigma}\right\}_{-\ln T-s_{i} \leq \sigma<\infty}$ defined in (5.2) can be parametrized as a multigraph

$$
Y_{s_{i}+\sigma}\left(y_{1}, y_{2}\right)=\boldsymbol{\Phi}_{s_{i}+\sigma}\left(y_{1}, y_{2}, v_{s_{i}}^{j}\left(y_{1}, y_{2}, \sigma\right)\right), \quad j=1, \cdots, m
$$

where $\boldsymbol{\Phi}_{s_{i}+\sigma}$ is the map in Definition 2.4 which parametrizes the tubular neighborhood of $\boldsymbol{\Gamma}_{s_{i}+\sigma}$ near $O$, and the functions satisfy

$$
\begin{gathered}
v_{s_{i}}^{1}(y, \sigma)<\cdots<v_{s_{i}}^{m}(y, \sigma), \\
v_{s_{i}}^{j}(y, \sigma) \rightarrow 0 \quad \text { away from } \bigcup_{-\infty<\sigma<\infty} e^{\frac{\sigma}{2}} \mathfrak{S}_{P} \times\{\sigma\} \text { for } j \in\{1, \cdots, m\} .
\end{gathered}
$$

In addition, let $v_{s_{i}}=v_{s_{i}}^{m}-v_{s_{i}}^{1}$, then it satisfies

$\partial_{\sigma} v_{s_{i}}=\partial_{k}\left(g_{s_{i}+\sigma}^{k l}\left(y, v_{s_{i}}^{m}, \nabla v_{s_{i}}^{m}\right) \partial_{l} v_{s_{i}}\right)+\left(-\frac{1}{2} y+b_{s_{i}}(y, \sigma)\right) \cdot \nabla v_{s_{i}}+\left(\frac{1}{2}+c_{s_{i}}(y, \sigma)\right) v_{s_{i}}$,

$$
\left.\partial_{2} v_{s_{i}}\right|_{y_{2}=0}=0
$$

in which the coefficients satisfy

$$
\begin{gathered}
g_{s_{i}+\sigma}^{k l}\left(y, v_{s_{i}}^{m}, \nabla v_{s_{i}}^{m}\right) \rightarrow \delta^{k l} \quad \text { and }\left.\quad g_{s_{i}+\sigma}^{12}\left(y, v_{s_{i}}^{m}, \nabla v_{s_{i}}^{m}\right)\right|_{y_{2}=0}=0, \\
\left|b_{s_{i}}\right|+\left|c_{s_{i}}\right| \rightarrow 0 .
\end{gathered}
$$

Furthermore, given $0<\varepsilon<1<\mathcal{T}<\infty$, for $i \gg 1$ there holds

$$
\begin{aligned}
& \sup _{0<\sigma \leq \mathcal{T}} \int_{\Pi \cap B_{(1-\varepsilon) \delta^{-1}}(O) \backslash \cup_{Q \in e^{\frac{\sigma}{2}} \mathfrak{S}_{P}} B_{(1+\varepsilon) \delta}(Q)} v_{s_{i}}(y, \sigma) d y \\
& \leq(1+\varepsilon) \int_{\Pi \cap B_{(1+\varepsilon) \delta^{-1}}(O) \backslash \cup_{Q \in e^{\frac{\sigma}{2}} \mathfrak{S}_{P}} B_{(1-\varepsilon) \delta}(Q)} v_{s_{i}}(y, 0) d y .
\end{aligned}
$$

Proof. Given $\delta>0$, by Proposition 4.3, Lemma 5.1 and Lemma 5.2, there exist a sequence $\left\{t_{i} \nearrow T\right\}$, a half plane $\Pi$ and a finite set $\mathfrak{S}_{P} \subset \Pi$, and an integer $m \in \mathbb{N}$ with the following properties. 
- Let $\lambda_{i}=\sqrt{T-t_{i}}$, then the sequence of MCF defined in (4.9) satisfies $\left\{\boldsymbol{\Sigma}_{\tau}^{(P, T), \lambda_{i}}\right\}_{-\frac{T}{\lambda_{i}^{2}} \leq \tau<0} \rightarrow\{\Pi\}_{-\infty<\tau<0}$ with finite multiplicity $m$ away from $\mathfrak{S}_{P} \times(-\infty, 0)$. The half plane $\Pi$ has free boundary on $\lim _{i \rightarrow \infty} \Gamma^{P, \lambda_{i}} \simeq \mathbb{R}^{2}$;

- Let $s_{i}=-\ln \left(T-t_{i}\right)$, then the sequence of normalized MCF defined in (5.2) satisfies $\left\{\Pi_{s_{i}+\sigma}\right\}_{-\ln T-s_{i} \leq \sigma<\infty} \rightarrow\{\Pi\}_{-\infty<\tau<\infty}$ with finite multiplicity $m$ away from $\underset{-\infty<\tau<\infty}{\cup} e^{\frac{\sigma}{2} \mathfrak{S}_{P}} \times\{\sigma\}$;

- The set $\boldsymbol{U}_{s_{i}}^{\delta}$ defined in (5.3) satisfies

$$
\sup _{0<\sigma \leq i} \mathcal{H}^{3}\left(\boldsymbol{U}_{s_{i}+\sigma}^{\delta}\right) \leq\left(1+\frac{1}{i}\right) \mathcal{H}^{3}\left(\boldsymbol{U}_{s_{i}}^{\delta}\right) .
$$

With out loss of generality, we may assume that

$$
\begin{gathered}
\lim _{i \rightarrow \infty} \boldsymbol{U}^{P, \lambda_{i}}=\left\{\left(x_{1}, x_{2}, x_{3}\right) \mid x_{1}, x_{3} \in \mathbb{R}, x_{2}>0\right\}, \\
\lim _{i \rightarrow \infty} \boldsymbol{\Gamma}^{P, \lambda_{i}}=\left\{\left(x_{1}, 0, x_{3}\right) \mid x_{1}, x_{3} \in \mathbb{R}\right\}, \\
\Pi=\left\{\left(x_{1}, x_{2}, 0\right) \mid x_{1} \in \mathbb{R}, x_{2} \geq 0\right\} .
\end{gathered}
$$

Let $\boldsymbol{\Phi}^{P, \lambda_{i}}$ be the map defined in Definition 2.4 which parametrizes the tubular neighborhood of $\boldsymbol{\Gamma}^{P, \lambda_{i}}$ near $O$ (see also Remark 2.5). Since $\boldsymbol{\Gamma}^{P, \lambda_{i}}$ satisfies $e^{-\frac{1}{2}\left(s_{i}+\sigma\right)} \boldsymbol{\kappa}$-graph condition, $\boldsymbol{\Phi}^{P, \lambda_{i}}$ converges to the identity map as $i \rightarrow \infty$. By (2.1) and the normal-vector-preserving property of $\boldsymbol{\Phi}^{P, \lambda_{i}}$, for each $-\infty<\tau<0$, $\left(\boldsymbol{\Phi}^{P, \lambda_{i}}\right)^{-1}\left(\boldsymbol{\Sigma}_{\tau}^{(P, T), \lambda_{i}}\right)$ has free boundary on

$$
\left(\boldsymbol{\Phi}^{P, \lambda_{i}}\right)^{-1}\left(\boldsymbol{\Gamma}^{P, \lambda_{i}}\right) \subset\left\{\left(y_{1}, 0, y_{3}\right) \mid y_{1}, y_{3} \in \mathbb{R}\right\}
$$

and it converges to

$$
\left\{\left(y_{1}, y_{2}, 0\right) \mid y_{1} \in \mathbb{R}, y_{2} \geq 0\right\} \simeq \Pi
$$

with multiplicity $m$ away from $\mathfrak{S}_{P}$. It follows that for $i \gg 1$ and away from $\mathfrak{S}_{P}$, $\left(\boldsymbol{\Phi}^{P, \lambda_{i}}\right)^{-1}\left(\boldsymbol{\Sigma}_{\tau}^{(P, T), \lambda_{i}}\right)$ is a disjoint union of graphs of $u_{\lambda_{i}}^{j}\left(y_{1}, y_{2}, t\right)$ defined on $\Pi$ for $j=1, \cdots, m$. We may assume that $u_{\lambda_{i}}^{1}(y, t)<\cdots<u_{\lambda_{i}}^{m}(y, t)$. Note that $\Theta_{\left\{\boldsymbol{\Sigma}_{t}\right\}}(P, T)>\frac{1}{2}$ implies $m>1$ (see Lemma 5.1), and that $u_{\lambda_{i}}^{j}(y, t) \rightarrow 0$ away from $\mathfrak{S}_{P} \times(-\infty, 0)$ for each $j \in\{1, \cdots, m\}$. Thus, we can parametrize $\boldsymbol{\Sigma}_{\tau}^{(P, T), \lambda_{i}}$ (away from $\mathfrak{S}_{P}$ ) as

$$
X_{\tau}^{(P, T), \lambda_{i}}\left(y_{1}, y_{2}\right)=\boldsymbol{\Phi}^{P, \lambda_{i}}\left(y_{1}, y_{2}, u_{\lambda_{i}}^{j}\left(y_{1}, y_{2}, t\right)\right), \quad j=1, \cdots, m .
$$

Using a similar argument as in Lemma 2.9, $u_{\lambda_{i}}^{j}(y, t)$ satisfies an analogous equation as (2.7). Namely,

$$
\partial_{\tau} u_{\lambda_{i}}^{j}=\boldsymbol{g}_{\lambda_{i}}^{k l}\left(y, u_{\lambda_{i}}^{j}, \nabla u_{\lambda_{i}}^{j}\right) \partial_{k l}^{2} u_{\lambda_{i}}^{j}+\boldsymbol{f}_{\lambda_{i}}\left(y, u_{\lambda_{i}}^{j}, \nabla u_{\lambda_{i}}^{j}\right),
$$

where $\boldsymbol{g}_{\lambda_{i}}^{k l}\left(y, u_{\lambda_{i}}^{j}, \nabla u_{\lambda_{i}}^{j}\right)$ and $\boldsymbol{f}_{\lambda_{i}}\left(y, u_{\lambda_{i}}^{j}, \nabla u_{\lambda_{i}}^{j}\right)$ are defined in the same way as (2.11) and (2.13) but with $\boldsymbol{\Phi}^{P, \lambda_{i}}$ in place of $\Phi$. More precisely, $\boldsymbol{g}_{\lambda_{i}}^{k l}\left(y, u_{\lambda_{i}}^{j}, \nabla u_{\lambda_{i}}^{j}\right)$ is the inverse of

$$
\boldsymbol{g}_{k l}^{\lambda_{i}}\left(y, u_{\lambda_{i}}^{j}, \nabla u_{\lambda_{i}}^{j}\right):=\partial_{k} X_{\tau}^{(P, T), \lambda_{i}} \cdot \partial_{l} X_{\tau}^{(P, T), \lambda_{i}}
$$


$=\boldsymbol{h}_{k l}^{\lambda_{i}}\left(y, u_{\lambda_{i}}^{j}\right)+\boldsymbol{h}_{k 3}^{\lambda_{i}}\left(y, u_{\lambda_{i}}^{j}\right) \partial_{l} u_{\lambda_{i}}^{j}+\boldsymbol{h}_{l 3}^{\lambda_{i}}\left(y, u_{\lambda_{i}}^{j}\right) \partial_{k} u_{\lambda_{i}}^{j}+\boldsymbol{h}_{33}^{\lambda_{i}}\left(y, u_{\lambda_{i}}^{j}\right) \partial_{k} u_{\lambda_{i}}^{j} \partial_{l} u_{\lambda_{i}}^{j}$, where

$$
\boldsymbol{h}_{k l}^{\lambda_{i}}\left(y_{1}, y_{2}, y_{3}\right)=\partial_{k} \boldsymbol{\Phi}^{P, \lambda_{i}}\left(y_{1}, y_{2}, y_{3}\right) \cdot \partial_{l} \boldsymbol{\Phi}^{P, \lambda_{i}}\left(y_{1}, y_{2}, y_{3}\right) \text {. }
$$

is the pull-back metric by $\boldsymbol{\Phi}^{P, \lambda_{i}}$ (see also (2.3) and (2.11)). Similarly, we define

$\boldsymbol{f}_{\lambda_{i}}\left(y, u_{\lambda_{i}}^{j}, \nabla u_{\lambda_{i}}^{j}\right)=\boldsymbol{g}_{\lambda_{i}}^{k l}\left(y, u_{\lambda_{i}}^{j}, \nabla u_{\lambda_{i}}^{j}\right)\left\{\boldsymbol{\Gamma}_{k l, \lambda_{i}}^{3}\left(y, u_{\lambda_{i}}^{j}\right)+\boldsymbol{Q}_{k l, \lambda_{i}}\left(y, u_{\lambda_{i}}^{j}, \nabla u_{\lambda_{i}}^{j}\right)\right\}$,

where $\boldsymbol{\Gamma}_{k l, \lambda_{i}}^{3}\left(y, u_{\lambda_{i}}^{j}\right)$ and $\boldsymbol{Q}_{k l, \lambda_{i}}\left(y, u_{\lambda_{i}}^{j}, \nabla u_{\lambda_{i}}^{j}\right)$ are defined analogously as (2.4) and (2.12) but with $\boldsymbol{\Phi}^{P, \lambda_{i}}$ and $\left(\Phi^{P, \lambda_{i}}, u_{\lambda_{i}}^{j}, \nabla u_{\lambda_{i}}^{j}\right)$ in place of $\Phi$ and $(\Phi, u, \nabla u)$, respectively. As in Lemma 2.9, we have

$$
\begin{gathered}
\left.\partial_{2} u_{\lambda_{i}}^{j}\right|_{y_{2}=0}=0, \\
\left.\boldsymbol{g}_{\lambda_{i}}^{12}\left(y, u_{\lambda_{i}}^{j}, \nabla u_{\lambda_{i}}^{j}\right)\right|_{y_{2}=0}=0 .
\end{gathered}
$$

Note that $\boldsymbol{\Gamma}^{P, \lambda_{i}}$ satisfies a $\lambda_{i} \kappa$-graph condition.

By (5.2) and Remark 2.5, $\Pi_{s_{i}+\sigma}$ can be parametrized as a multigraph

$$
Y_{s_{i}+\sigma}(y)=\boldsymbol{\Phi}_{s_{i}+\sigma}\left(y, v_{s_{i}}^{j}(y, \sigma)\right), \quad j=1, \cdots, m,
$$

where

$$
v_{s_{i}}^{j}(y, \sigma)=\left.\frac{1}{\sqrt{-\tau}} u_{\lambda_{i}}^{j}(\sqrt{-\tau} y, \tau)\right|_{\tau=-e^{-\sigma}}
$$

and $\boldsymbol{\Phi}_{s_{i}+\sigma}$ is the map defined in 2.4 (which parametrizes a tubular neighborhood of $\left.\boldsymbol{\Gamma}_{s_{i}+\sigma}\right)$. Note that $v_{s_{i}}^{1}(y, \sigma)<\cdots<v_{s_{i}}^{m}(y, \sigma)$ and $v_{s_{i}}^{j}(y, \sigma) \rightarrow 0$ away from $\left\{(y, \sigma) \mid y \in e^{\frac{\sigma}{2}} \mathfrak{S}_{P}, \sigma \in \mathbb{R}\right\}$. By (5.8), (5.9) and (5.11), we have

$$
\begin{aligned}
\partial_{\sigma} v_{s_{i}}^{j}+\frac{1}{2} y \cdot \nabla v_{s_{i}}^{j}-\frac{1}{2} v_{s_{i}}^{j}= & \boldsymbol{g}_{s_{i}+\sigma}^{k l}\left(y, v_{s_{i}}^{j}, \nabla v_{s_{i}}^{j}\right) \partial_{k l}^{2} v_{s_{i}}^{j}+\boldsymbol{f}_{s_{i}+\sigma}\left(y, v_{s_{i}}^{j}, \nabla v_{s_{i}}^{j}\right), \\
& \left.\partial_{2} v_{s_{i}}^{j}\right|_{y_{2}=0}=0,
\end{aligned}
$$

for $j=1, \cdots, m$, where $\boldsymbol{g}_{s_{i}+\sigma}^{k l}\left(y, v_{s_{i}}^{j}, \nabla v_{s_{i}}^{j}\right)$ and $\boldsymbol{f}_{s_{i}+\sigma}\left(y, v_{s_{i}}^{j}, \nabla v_{s_{i}}^{j}\right)$ are defined analogously as (2.11) and (2.13) but with $\boldsymbol{\Phi}_{s_{i}+\sigma}$ in place of $\Phi$. Note that $\boldsymbol{\Gamma}_{s_{i}+\sigma}$ satisfies our $e^{-\frac{s_{i}+\sigma}{2}} \kappa$-graph condition.

Next, let $v_{s_{i}}=v_{s_{i}}^{m}-v_{s_{i}}^{1}$. By (5.12), we get

$$
\begin{gathered}
\partial_{\sigma} v_{s_{i}}+\frac{1}{2} y \cdot \nabla v_{s_{i}}-\frac{1}{2} v_{s_{i}}=\partial_{k}\left(\boldsymbol{g}_{s_{i}+\sigma}^{k l}\left(y, v_{s_{i}}^{m}, \nabla v_{s_{i}}^{m}\right) \partial_{l} v_{s_{i}}\right)+\Xi_{s_{i}+\sigma}, \\
\left.\partial_{2} v_{s_{i}}\right|_{y_{2}=0}=0,
\end{gathered}
$$

where

$$
\begin{gathered}
\Xi_{s_{i}+\sigma}=-\partial_{k}\left(\boldsymbol{g}_{s_{i}+\sigma}^{k l}\left(y, v_{s_{i}}^{m}, \nabla v_{s_{i}}^{m}\right)\right) \partial_{l} v_{s_{i}} \\
+\left(\boldsymbol{g}_{s_{i}+\sigma}^{k l}\left(y, v_{s_{i}}^{m}, \nabla v_{s_{i}}^{m}\right)-\boldsymbol{g}_{s_{i}+\sigma}^{k l}\left(y, v_{s_{i}}^{1}, \nabla v_{s_{i}}^{1}\right)\right) \partial_{k l}^{2} v_{s_{i}}^{1} \\
+\boldsymbol{f}_{s_{i}+\sigma}\left(y, v_{s_{i}}^{m}, \nabla v_{s_{i}}^{m}\right)-\boldsymbol{f}_{s_{i}+\sigma}\left(y, v_{s_{i}}^{1}, \nabla v_{s_{i}}^{1}\right) .
\end{gathered}
$$

From (2.13), we have

$$
\Xi_{s_{i}+\sigma}=b_{s_{i}}(y, \sigma) \cdot \nabla v_{s_{i}}+c_{s_{i}}(y, \sigma) v_{s_{i}} .
$$

with the vector-valued function $b_{s_{i}}$ and the scalar function $c_{s_{i}}$ satisfying

$$
\left|b_{s_{i}}\right|+\left|c_{s_{i}}\right|
$$


$\leq C\left(\left\|\boldsymbol{\varphi}_{s_{i}+\sigma}\right\|_{C^{3}}, y_{2}, \nabla v_{s_{i}}^{m}, \nabla v_{s_{i}}^{1}\right)\left(\left\|v_{s_{i}}^{m}\right\|_{C^{2}}+\left\|v_{s_{i}}^{1}\right\|_{C^{2}}+\left\|\boldsymbol{\Gamma}_{k l, s_{i}+\sigma}^{p}\right\|_{L^{\infty}}+\left[\boldsymbol{\Gamma}_{k l, s_{i}+\sigma}^{p}\right]_{1, \text { spacial }}\right)$, where $\boldsymbol{\varphi}_{s_{i}+\sigma}$ is the local graph of $\boldsymbol{\Gamma}_{s_{i}+\sigma}$ (see Definition 2.3), $\boldsymbol{\Gamma}_{k l, s_{i}+\sigma}^{p}$ is the connection associated with $\boldsymbol{\Phi}_{s_{i}+\sigma}$ (see (2.4) ), and $[\cdot]_{1, \text { spacial }}$ is the Lipschitz norm with respect to the spacial variable (see Lemma 2.9). Note that (5.10) yields

$$
\left.\boldsymbol{g}_{s_{i}+\sigma}^{12}\left(y, v_{s_{i}}^{m}, \nabla v_{s_{i}}^{m}\right)\right|_{y_{2}=0}=0
$$

Since $\boldsymbol{\Gamma}_{s_{i}+\sigma}$ satisfies $e^{-\frac{1}{2}\left(s_{i}+\sigma\right)} \boldsymbol{\kappa}$-graph condition, we have $\boldsymbol{\Phi}_{s_{i}+\sigma}(Y) \stackrel{C^{3}}{\rightarrow} Y$ as $i \rightarrow$ $\infty$. It follows that

$$
\boldsymbol{g}_{s_{i}+\sigma}^{k l}\left(y, v_{s_{i}}^{m}, \nabla v_{s_{i}}^{m}\right) \rightarrow \boldsymbol{\delta}^{k l}, \quad\left|b_{s_{i}}^{k}\right|+\left|c_{s_{i}}\right| \rightarrow 0 .
$$

Moreover, the push-forward measure $\boldsymbol{\Phi}_{s_{i}+\sigma}^{*} \mathcal{H}^{3}$ converge to $\mathcal{H}^{3}$ as $i \rightarrow \infty$. Namely, for every Borel set $\mathcal{B}$ in $\mathbb{R}^{3}$, there holds

$$
\mathbf{\Phi}_{s_{i}+\sigma}^{*} \mathcal{H}^{3}(\mathcal{B})=\mathcal{H}^{3}\left(\Phi_{s_{i}+\sigma}^{-1}(\mathcal{B})\right) \rightarrow \mathcal{H}^{3}(\mathcal{B}) .
$$

Lastly, given $0<\varepsilon<1<\mathcal{T}<\infty$, by (5.3), for $i \gg 1$ we have

$$
\begin{gathered}
\sup _{|\sigma| \leq \mathcal{T}} \int_{\Pi \cap B_{(1-\varepsilon) \delta^{-1}}(O) \backslash \cup_{Q \in e^{\frac{\sigma}{2}} \mathfrak{S}_{P}} B_{(1+\varepsilon) \delta}(Q)} v_{s_{i}}(y, \sigma) d y \leq\left(1+\frac{\varepsilon}{2}\right) \mathcal{H}^{3}\left(\boldsymbol{U}_{s_{i}+\sigma}^{\delta}\right), \\
\int_{\Pi \cap B_{(1+\varepsilon) \delta^{-1}}(O) \backslash \cup_{Q \in e^{\frac{\sigma}{2}} \mathfrak{S}_{P}} B_{(1-\varepsilon) \delta}(Q)} v_{s_{i}}(y, 0) d y \geq\left(1-\frac{\varepsilon}{2}\right) \mathcal{H}^{3}\left(\boldsymbol{U}_{s_{i}}^{\delta}\right) .
\end{gathered}
$$

Using (5.7), we get

for $i \gg 1$.

$$
\begin{gathered}
\sup _{0<\sigma \leq \mathcal{T}} \int_{\Pi \cap B_{(1-\varepsilon) \delta^{-1}}(O) \backslash \cup_{Q \in e^{\frac{\sigma}{2}} \mathfrak{G}_{P}} B_{(1+\varepsilon) \delta}(Q)} v_{s_{i}}(y, \sigma) d y \\
\leq \frac{1+\frac{\varepsilon}{2}}{1-\frac{\varepsilon}{2}}\left(1+\frac{1}{i}\right) \int_{\Pi \cap B_{(1+\varepsilon) \delta^{-1}}(O) \backslash \cup_{Q \in e^{\frac{\sigma}{2}} \mathfrak{S}_{P}} B_{(1-\varepsilon) \delta}(Q)} v_{s_{i}}(y, 0) d y \\
\leq(1+\varepsilon) \int_{\Pi \cap B_{(1+\varepsilon) \delta^{-1}}(O) \backslash \cup_{Q \in e^{\frac{\sigma}{2}} \mathfrak{S}_{P}} B_{(1-\varepsilon) \delta}(Q)} v_{s_{i}}(y, 0) d y
\end{gathered}
$$

What follows are the estimates on the upper and lower bound for the difference function. Since the function is defined on a half-plane, we first use the method of reflection and then use the equation satisfied by the extension to derive the estimates.

Lemma 5.4. Let $\delta>0$ and $\left\{v_{s_{i}}\right\}$ be as in Proposition 5.3. Define $\bar{v}_{s_{i}}$ as the even extension of $v_{s_{i}}$, i.e.

$$
\bar{v}_{s_{i}}(y, t)=\left\{\begin{array}{l}
v_{s_{i}}(y, \sigma), y_{2} \geq 0 \\
v_{s_{i}}(\tilde{y}, \sigma), y_{2}<0
\end{array},\right.
$$

where $\tilde{y}=\widetilde{\left(y_{1}, y_{2}\right)}=\left(y_{1},-y_{2}\right)$. Then $\bar{v}_{s_{i}}$ is $C^{2}$ away from $\underset{\sigma \in \mathbb{R}}{\cup} e^{\frac{\sigma}{2}}\left(\overline{\mathfrak{S}_{P}}\right) \times\{\sigma\}$, where $\overline{\mathfrak{S}_{P}}=\mathfrak{S}_{P} \cup \widetilde{\mathfrak{S}_{P}}$, and it satisfies

$$
\partial_{\sigma} \bar{v}_{s_{i}}=\partial_{k}\left(\overline{\boldsymbol{g}}_{s_{i}}^{k l}(y, \sigma) \partial_{l} \bar{v}_{s_{i}}\right)+\left(-\frac{1}{2} y+\bar{b}_{s_{i}}(y, \sigma)\right) \cdot \nabla v_{s_{i}}+\left(\frac{1}{2}+\bar{c}_{s_{i}}(y, \sigma)\right) v_{s_{i}}
$$


where

$$
\begin{gathered}
\overline{\boldsymbol{g}}_{s_{i}}^{k l}(y, \sigma)=\left\{\begin{array}{c}
\boldsymbol{g}_{s_{i}+\sigma}^{k l}\left(y, v_{s_{i}}^{m}, \nabla v_{s_{i}}^{m}\right), y_{2} \geq 0 \\
(-1)^{k+l} \boldsymbol{g}_{s_{i}+\sigma}^{k l}\left(\tilde{y}, v_{s_{i}}^{m}(\tilde{y}, \sigma), \nabla v_{s_{i}}^{m}(\tilde{y}, \sigma)\right), y_{2}<0
\end{array}, \quad \text { for } k, l \in\{1,2\} ;\right. \\
\bar{b}_{s_{i}}^{k}(y, \sigma)=\left\{\begin{array}{c}
b_{s_{i}}^{k}(y, \sigma), y_{2} \geq 0 \\
(-1)^{k+1} b_{s_{i}}^{k}(\tilde{y}, \sigma) y_{2}<0
\end{array}, \text { for } k \in\{1,2\} ;\right. \\
\bar{c}_{s_{i}}(y, \sigma)=\left\{\begin{array}{c}
c_{s_{i}}(y, \sigma), y_{2} \geq 0 \\
c_{s_{i}}(\tilde{y}, \sigma) y_{2}<0
\end{array} .\right.
\end{gathered}
$$

The functions $\overline{\boldsymbol{g}}_{s_{i}}^{k l}(y, \sigma), \bar{c}_{s_{i}}(y, \sigma)$ are Lipschitz in $y, \bar{b}_{s_{i}} \in L^{\infty}$ and

$$
\overline{\boldsymbol{g}}_{s_{i}}^{k l} \rightarrow \boldsymbol{\delta}^{k l}, \quad\left|\bar{b}_{s_{i}}\right|+\left|\bar{c}_{s_{i}}\right| \rightarrow 0
$$

Additionally, let

$$
\sigma_{\delta}=\max \left\{-2 \ln \left(\frac{\delta}{2} \min _{Q \in \mathfrak{S}_{P} \backslash\{O\}}|Q|\right), 1\right\}
$$

and fix $Q_{\delta} \in \Pi \cap B_{\frac{1}{2} \delta^{-1}}(O) \backslash B_{\frac{3}{2} \delta}(O)$. Then for any given $\mathcal{T}>\sigma_{\delta}+1$, if $i \gg 1$, we have

- For $y \in B_{\frac{2}{3} \delta^{-1}}(O) \backslash B_{\frac{4}{3} \delta}(O), \sigma_{\delta}+1 \leq \sigma \leq \mathcal{T}$, there holds

$$
C(\delta, \mathcal{T})^{-1} \bar{v}_{s_{i}}\left(Q_{\delta}, \sigma_{\delta}\right) \leq \bar{v}_{s_{i}}(y, \sigma) \leq C(\delta) \bar{v}_{s_{i}}\left(Q_{\delta}, \sigma_{\delta}\right)
$$

- For $y \in B_{\frac{2}{3} \delta^{-1}}(O) \backslash B_{\frac{4}{3} \delta}(O), \sigma_{\delta}+1 \leq \sigma \leq \sigma_{\delta}+2$, there holds

$$
\bar{v}_{s_{i}}(y, \sigma) \geq C(\delta)^{-1} \bar{v}_{s_{i}}\left(Q_{\delta}, \sigma_{\delta}\right) .
$$

Proof. The first part follows from (5.4), (5.5) and the reflection principle (see Lemma 2.10). For the second part, note that $\sigma_{\delta} \geq 1$ is chosen so that

$$
e^{\frac{\sigma_{\delta}}{2}} \min _{Q \in \mathfrak{S}_{P} \backslash\{O\}}|Q| \geq 2 \delta^{-1}
$$

if $\mathfrak{S}_{P} \backslash\{O\} \neq \emptyset$. In particular, $v_{s_{i}}(\cdot, \sigma)$ is $C^{2}$ in $B_{2 \delta^{-1}}(O)$ away from $O$ for $\sigma \geq \sigma_{\delta}$ and $i \gg 1$.

Given $\mathcal{T}>\sigma_{\delta}+1$, by (5.13) $i \gg 1$ we have

- For $y \in B_{\frac{2}{3} \delta^{-1}}(O) \backslash B_{\frac{4}{3} \delta}(O), \sigma_{\delta}+1 \leq \sigma \leq \mathcal{T}$,

$$
\bar{v}_{s_{i}}(\cdot, \sigma) \geq C(\delta, \mathcal{T})^{-1} \bar{v}_{s_{i}}\left(Q_{\delta}, \sigma_{\delta}\right)
$$

- For $y \in B_{\frac{2}{3} \delta^{-1}}(O) \backslash B_{\frac{4}{3} \delta}(O), \sigma_{\delta}+1 \leq \sigma \leq \sigma_{\delta}+2$,

$$
\bar{v}_{s_{i}}(\cdot, \sigma) \geq C(\delta)^{-1} \bar{v}_{s_{i}}\left(Q_{\delta}, \sigma_{\delta}\right)
$$

- For $y \in B_{\frac{5}{4} \delta^{-1}}(O) \backslash \cup_{Q \in \mathfrak{S}_{P}} B_{\frac{3}{4} \delta}(Q)$,

$$
\bar{v}_{s_{i}}(\cdot, 0) \leq C(\delta) \bar{v}_{s_{i}}\left(Q_{\delta}, \sigma_{\delta}\right) .
$$

In particular, the last one yields

$$
\int_{B_{\frac{5}{4} \delta^{-1}}(O) \backslash \cup_{Q \in \mathfrak{S}_{P}} B_{\frac{3}{4} \delta}(Q)} \bar{v}_{s_{i}}(y, 0) d y \leq C(\delta) \bar{v}_{s_{i}}\left(Q_{\delta}, \sigma_{\delta}\right) .
$$

To derive the upper bound for $\bar{v}_{s_{i}}$, we first use (5.6) and the above inequality to get

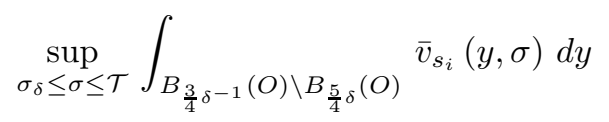




$$
\lesssim \int_{\Pi \cap B_{\frac{5}{4} \delta^{-1}}(O) \backslash B_{\frac{3}{4} \delta}(O)} \bar{v}_{s_{i}}(y, 0) d y \leq C(\delta) \bar{v}_{s_{i}}\left(Q_{\delta}, \sigma_{\delta}\right) .
$$

By (5.13), (5.14) and the mean value inequality (cf. [AS]), for $y \in B_{\frac{2}{3} \delta^{-1}}(O) \backslash$ $B_{\frac{4}{3} \delta}(O), \sigma_{\delta}+1 \leq \sigma \leq \mathcal{T}$, we have

$$
\begin{gathered}
\bar{v}_{s_{i}}\left(y_{0}, \sigma_{0}\right) \lesssim f_{B_{\frac{\delta}{12}}\left(y_{0}\right) \times\left(\sigma_{0}-\left(\frac{\delta}{12}\right)^{2}, \sigma\right)} \bar{v}_{s_{i}}(y, \sigma) d y d \sigma \\
\lesssim \frac{1}{\delta^{2}} \sup _{\sigma_{0}-\left(\frac{\delta}{12}\right)^{2}<\sigma<\sigma_{0}} \int_{B_{\frac{3}{4} \delta^{-1}}(O) \backslash B_{\frac{5}{4} \delta}(O)} \bar{v}_{s_{i}}(y, \sigma) d y \leq C(\delta) \bar{v}_{s_{i}}\left(Q_{\delta}, \sigma_{\delta}\right) .
\end{gathered}
$$

Now we are in a position to prove the unity of Huisken's density. Our proof follows closely the arguments in [LW] (see also [CM]).

Proposition 5.5. (Unity of Huisken's Density)

Let $P$ be a limit point of $\left\{\boldsymbol{\Sigma}_{t}\right\}_{0 \leq t<T}$ as $t \nearrow T$. Then

$$
\Theta_{\left\{\boldsymbol{\Sigma}_{t}\right\}}(P, T)=\left\{\begin{array}{ll}
1, & P \in \boldsymbol{U} \\
\frac{1}{2}, & P \in \boldsymbol{\Gamma}
\end{array} .\right.
$$

Proof. We will focus on the case where $P \in \boldsymbol{\Gamma}$ since the argument for $P \in \boldsymbol{U}$ is similar.

Note that the mean convexity of $\boldsymbol{\Gamma}$ yields $\Theta_{\left\{\boldsymbol{\Sigma}_{t}\right\}}(P, T) \geq \frac{1}{2}$ (cf. [K]). Suppose that $\Theta_{\left\{\boldsymbol{\Sigma}_{t}\right\}}(P, T)>\frac{1}{2}$, then from Proposition 3.10, Proposition 2.12 and Lemma 2.7. we know that $P \in \mathcal{S}$ (see Section 4). Below we will derive a contradiction in three steps and hence prove the proposition.

Step 1: Prove that

$$
\int_{\mathbb{R}^{2}}\left(|\nabla \eta|^{2}-\frac{1}{2} \eta^{2}\right) e^{-\frac{|y|^{2}}{4}} d y \geq 0
$$

for any function $\eta\left(y_{1}, y_{2}\right) \in C_{c}\left(\mathbb{R}^{2}\right) \cap W^{1,2}\left(\mathbb{R}^{2}\right)$ satisfying $\eta(0,0)=0$.

Proof of Step 1. By approximation, it suffices to show the following. Given $0<\delta<1$ and function $\eta\left(y_{1}, y_{2}\right) \in C_{c}^{1}\left(B_{\frac{1}{2} \delta^{-1}}(O) \backslash B_{\frac{3}{2} \delta}(O)\right)$, there holds

$$
\int_{\mathbb{R}^{2}}\left(|\nabla \eta|^{2}-\frac{1}{2} \eta^{2}\right) e^{-\frac{|y|^{2}}{4}} d y \geq 0
$$

For that purpose, let $\left\{\bar{v}_{s_{i}}\right\}$ be the sequence of functions in Lemma 5.4. Define

By (5.13), we get

$$
w_{s_{i}}(y, \sigma)=\frac{\bar{v}_{s_{i}}(y, \sigma)}{\bar{v}_{s_{i}}\left(Q_{\delta}, \sigma_{\delta}\right)} .
$$

$$
\partial_{\sigma} w_{s_{i}}=\partial_{k}\left(\overline{\boldsymbol{g}}_{s_{i}}^{k l}(y, \sigma) \partial_{l} w_{s_{i}}\right)+\left(-\frac{1}{2} y+\bar{b}_{s_{i}}(y, \sigma)\right) \cdot \nabla w_{s_{i}}+\left(\frac{1}{2}+\bar{c}_{s_{i}}(y, \sigma)\right) w_{s_{i}}
$$

Note that $w_{s_{i}}\left(y_{1}, y_{2}, \sigma\right)$ is an even function in $y_{2}$. For $i \gg 1$, Lemma 5.4 implies that

$$
C(\delta, \sigma)^{-1} \leq w_{s_{i}}(y, \sigma) \leq C(\delta)
$$

for $y \in B_{\frac{2}{3} \delta^{-1}}(O) \backslash B_{\frac{4}{3} \delta}(O), \sigma \geq \sigma_{\delta}+1$, and

$$
w_{s_{i}}(y, \sigma) \geq C(\delta)^{-1}
$$


for $y \in B_{\frac{2}{3} \delta^{-1}}(O) \backslash B_{\frac{4}{3} \delta}(O), \sigma_{\delta}+1 \leq \sigma \leq \sigma_{\delta}+2$. By (5.14) and Hölder estimates (cf. [AS]), there exists $0<\alpha<1$ (which is independent of $s_{i}$ ) so that

$$
\left[w_{s_{i}}\right]_{\alpha} \lesssim \frac{1}{\delta^{\alpha}}\left\|w_{s_{i}}\right\|_{L^{\infty}} \leq C(\delta)
$$

Consequently, there exists a positive function $w(y, \sigma)$ so that, after passing to a subsequence, $w_{s_{i}}$ converges locally uniformly to $w$ (by Arzelà-Ascoli theorem) and $\nabla w_{s_{i}}$ converges weakly in $L_{l o c}^{2}$ to $\nabla w$ (by Caccioppoli estimate) on $y \in$ $\left(B_{\frac{1}{2} \delta^{-1}}(O) \backslash B_{\frac{3}{2} \delta}(O)\right), \sigma \geq \sigma_{\delta}+2$ as $i \rightarrow \infty$. Note that $w\left(y_{1}, y_{1}, \sigma\right)$ is even in $y_{2}$ and satisfies

$$
C(\delta, \sigma)^{-1} \leq w(y, \sigma) \leq C(\delta)
$$

for $y \in B_{\frac{1}{2} \delta^{-1}}(O) \backslash B_{\frac{3}{2} \delta}(O), \sigma \geq \sigma_{\delta}+1$, and

$$
w(y, \sigma) \geq C(\delta)^{-1}
$$

for $y \in B_{\frac{1}{2} \delta^{-1}}(O) \backslash B_{\frac{3}{2} \delta}(O), \sigma_{\delta}+1 \leq \sigma \leq \sigma_{\delta}+2$.

It follows that $\ln w_{s_{i}}$ converges locally uniformly to $\ln w$, and $\nabla \ln w_{s_{i}}$ converges weakly in $L_{l o c}^{2}$ to $\nabla \ln w$ as $i \rightarrow \infty$. Additionally, by taking the logarithm of the equation for $w_{s_{i}}$, we have

$$
\begin{gathered}
\partial_{\sigma} \ln w_{s_{i}}-\partial_{k}\left(\overline{\boldsymbol{g}}_{s_{i}}^{k l}(y, \sigma) \partial_{l} \ln w_{s_{i}}\right) \\
=\overline{\boldsymbol{g}}_{s_{i}}^{k l}(y, \sigma) \partial_{k} \ln w_{s_{i}} \partial_{l} \ln w_{s_{i}}+\left(-\frac{1}{2} y+\bar{b}_{s_{i}}(y, \sigma)\right) \cdot \nabla \ln w_{s_{i}}+\left(\frac{1}{2}+\bar{c}_{s_{i}}(y, \sigma)\right) .
\end{gathered}
$$

Multiplication of the above equation by $\eta^{2}(y) e^{-\frac{|y|^{2}}{4}}$ and integration gives

$$
\begin{gathered}
\left.\int_{\mathbb{R}^{2}} \ln w_{s_{i}}(y, \sigma) \eta^{2}(y) e^{-\frac{|y|^{2}}{4}} d y\right|_{\sigma=\sigma_{\delta}+2} ^{\mathcal{T}}+\int_{\sigma_{\delta}+2}^{\mathcal{T}} \int_{\mathbb{R}^{2}} \overline{\boldsymbol{g}}_{s_{i}}^{k l}(y, \sigma) \partial_{l} \ln w_{s_{i}}\left(\partial_{k} \eta^{2}-\frac{1}{2} y_{k} \eta^{2}\right) e^{-\frac{|y|^{2}}{4}} d y d \sigma \\
=\int_{\sigma_{\delta}+2}^{\mathcal{T}} \int_{\mathbb{R}^{2}} \overline{\boldsymbol{g}}_{s_{i}}^{k l}(y, \sigma) \partial_{k} \ln w_{s_{i}} \partial_{l} \ln w_{s_{i}} \eta^{2}(y) e^{-\frac{|y|^{2}}{4}} d y d \sigma \\
+\int_{\sigma_{\delta}+2}^{\mathcal{T}} \int_{\mathbb{R}^{2}}\left\{\left(-\frac{1}{2} y+\bar{b}_{s_{i}}(y, \sigma)\right) \cdot \nabla \ln w_{s_{i}}+\left(\frac{1}{2}+\bar{c}_{s_{i}}(y, \sigma)\right)\right\} \eta^{2}(y) e^{-\frac{|y|^{2}}{4}} d y d \sigma
\end{gathered}
$$

for any $\sigma_{\delta}+2<\mathcal{T}<\infty$. Letting $i \rightarrow \infty$ gives

$$
\begin{aligned}
& \left.\int_{\mathbb{R}^{2}} \ln w(y, \sigma) \eta^{2} e^{-\frac{|y|^{2}}{4}} d y\right|_{\sigma=\sigma_{\delta}+2} ^{\mathcal{T}}+\int_{\sigma_{\delta}+2}^{\mathcal{T}} \int_{\mathbb{R}^{2}} \nabla \ln w \cdot\left(\nabla \eta^{2}-\frac{1}{2} y \eta^{2}\right) e^{-\frac{|y|^{2}}{4}} d y d \sigma \\
& =\int_{\sigma_{\delta}+2}^{\mathcal{T}} \int_{\mathbb{R}^{2}}|\nabla \ln w|^{2} \eta^{2} e^{-\frac{|y|^{2}}{4}} d y d \sigma+\int_{\sigma_{\delta}+2}^{\mathcal{T}} \int_{\mathbb{R}^{2}}\left\{-\frac{1}{2} y \cdot \nabla \ln w+\frac{1}{2}\right\} \eta^{2} e^{-\frac{|y|^{2}}{4}} d y d \sigma .
\end{aligned}
$$

After completing the square, we get

It follows that

$$
\begin{gathered}
\int_{\mathbb{R}^{2}} \ln \frac{w(y, \mathcal{T})}{w\left(y, \sigma_{\delta}+2\right)} \eta^{2} e^{-\frac{|y|^{2}}{4}} d y+\int_{\sigma_{\delta}+2}^{\mathcal{T}} \int_{\mathbb{R}^{2}}\left(|\nabla \eta|^{2}-\frac{1}{2} \eta^{2}\right) e^{-\frac{|y|^{2}}{4}} d y d \sigma \\
=\int_{\sigma_{\delta}+2}^{\mathcal{T}} \int_{\mathbb{R}^{2}}|\nabla \eta-\eta \nabla \ln w|^{2} e^{-\frac{|y|^{2}}{4}} d y d \sigma
\end{gathered}
$$

$$
\int_{\mathbb{R}^{2}}\left(|\nabla \eta|^{2}-\frac{1}{2} \eta^{2}\right) e^{-\frac{|y|^{2}}{4}} d y \geq \frac{1}{\mathcal{T}-\left(\sigma_{\delta}+2\right)} \int_{\mathbb{R}^{2}} \ln \frac{w\left(y, \sigma_{\delta}+2\right)}{w(y, \mathcal{T})} \eta^{2} e^{-\frac{|y|^{2}}{4}} d y
$$




$$
=\int_{\mathbb{R}^{2}} \ln \left(\frac{w\left(y, \sigma_{\delta}+2\right)}{w(y, \mathcal{T})}\right)^{\frac{1}{\mathcal{T}-\left(\sigma_{\delta}+2\right)}} \eta^{2} e^{-\frac{|y|^{2}}{4}} d y .
$$

By (5.15) and (5.16), we have

$$
\left(\frac{w\left(y, \sigma_{\delta}+2\right)}{w(y, \mathcal{T})}\right)^{\frac{1}{\mathcal{T}-\left(\sigma_{\delta}+2\right)}} \geq C(\delta)^{-\frac{1}{\mathcal{T}-\left(\sigma_{\delta}+2\right)}} \rightarrow 1 \quad \text { as } \quad \mathcal{T} \nearrow \infty
$$

Thus, we have $\int_{\mathbb{R}^{2}}\left(|\nabla \eta|^{2}-\frac{1}{2} \eta^{2}\right) e^{-\frac{|y|^{2}}{4}} d y \geq 0$. Q.E.D.

Step 2: Prove that

$$
\int_{\mathbb{R}^{2}}\left(|\nabla \eta|^{2}-\frac{1}{2} \eta^{2}\right) e^{-\frac{|y|^{2}}{4}} d y \geq 0
$$

for any function $\eta\left(y_{1}, y_{2}\right) \in C_{c}\left(\mathbb{R}^{2}\right) \cap W^{1,2}\left(\mathbb{R}^{2}\right)$. Note that $\eta$ does not need to vanish at $(0,0)$ as in Step 1.

Proof of Step 2. By approximation, it suffices to show the following. Given a function $\eta\left(y_{1}, y_{2}\right) \in C_{c}^{1}\left(\mathbb{R}^{2}\right)$, there holds

$$
\int_{\mathbb{R}^{2}}\left(|\nabla \eta|^{2}-\frac{1}{2} \eta^{2}\right) e^{-\frac{|y|^{2}}{4}} d y \geq 0 .
$$

To achieve that, for every $0<\delta \ll 1$, let

$$
\psi_{\delta}(\xi)=2 \delta \xi^{2 \delta-1} \quad \forall 0<\xi<1 .
$$

By a simple calculation, we have

$$
\int_{0}^{1} \psi_{\delta}(\xi) d \xi=1 \quad \text { and } \quad \int_{0}^{1} \psi_{\delta}^{2}(\xi) \xi d \xi=\delta .
$$

Now define

$$
\zeta_{\delta}(r)=\left\{\begin{array}{c}
\int_{0}^{r} \frac{1}{\delta} \psi_{\delta}\left(\frac{\rho}{\delta}\right) d \rho, \quad 0 \leq r \leq \delta \\
1, \quad r>\delta
\end{array} .\right.
$$

Notice that $\zeta_{\delta}(r) \in C[0, \infty), \zeta_{\delta}(0)=0$,

$$
\int_{0}^{\infty}\left|\zeta_{\delta}^{\prime}(r)\right|^{2} r d r=\int_{0}^{\delta} \frac{1}{\delta^{2}} \psi_{\delta}^{2}\left(\frac{r}{\delta}\right) r d r=\delta,
$$

and $\zeta_{\delta} \rightarrow 1$ as $\delta \searrow 0$.

Next, let's define

$$
\eta_{\delta}(y)=\zeta_{\delta}(|y|) \eta(y) .
$$

Then $\eta_{\delta} \in C_{c}\left(\mathbb{R}^{2}\right) \cap W^{1,2}\left(\mathbb{R}^{2}\right)$ and $\eta_{\delta}(0,0)=0$. It follows from Step 1 that

$$
\int_{\mathbb{R}^{2}}\left(\left|\nabla \eta_{\delta}\right|^{2}-\frac{1}{2} \eta_{\delta}^{2}\right) e^{-\frac{|y|^{2}}{4}} d y \geq 0 .
$$

Note that

$$
\nabla \eta_{\delta}(y)=\zeta_{\delta}(|y|) \nabla \eta(y)+\zeta_{\delta}^{\prime}(|y|) \frac{y}{|y|} \eta(y)
$$

and that

$$
\int_{\mathbb{R}^{2}}\left|\zeta_{\delta}^{\prime}(|y|) \eta(y)\right|^{2} e^{-\frac{|y|^{2}}{4}} d y \leq 2 \pi\|\eta\|_{L^{\infty}}^{2} \int_{0}^{\infty}\left|\zeta_{\delta}^{\prime}(r)\right|^{2} r d r=2 \pi\|\eta\|_{L^{\infty}}^{2} \delta .
$$

Letting $\delta \searrow 0$ gives $\int_{\mathbb{R}^{2}}\left(|\nabla \eta|^{2}-\frac{1}{2} \eta^{2}\right) e^{-\frac{|y|^{2}}{4}} d y \geq 0$. Q.E.D. 
Step 3: For each $R>0$, let

$$
\eta_{R}(y)=\left\{\begin{array}{cc}
1, & |y| \leq R \\
R+1-|y|, & R<|y| \leq R+1 . \\
0, & |y|>R+1
\end{array} .\right.
$$

Then we have

$$
\int_{\mathbb{R}^{2}}\left(\left|\nabla \eta_{R}\right|^{2}-\frac{1}{2} \eta_{R}^{2}\right) e^{-\frac{|y|^{2}}{4}} d y \rightarrow-\frac{1}{2} \int_{\mathbb{R}^{2}} e^{-\frac{|y|^{2}}{4}} d y=-2 \pi
$$

as $R \rightarrow \infty$. However, by Step 2 we should have

$$
\int_{\mathbb{R}^{2}}\left(\left|\nabla \eta_{R}\right|^{2}-\frac{1}{2} \eta_{R}^{2}\right) e^{-\frac{|y|^{2}}{4}} d y \geq 0 \quad \forall R>0 .
$$

Thus, we get the desired contradiction.

Thanks to Proposition[5.5, Allard's regularity theorem (cf. [Al]) and Proposition 3.7, now we can improve Proposition 4.3 as follows.

Corollary 5.6. Given a sequence $\left\{\lambda_{i} \searrow 0\right\}_{i \in \mathbb{N}}$, there exist a half plane $\Pi$ so that the a subsequence of (4.9) converges to $\{\Pi\}_{-\infty<\tau<0}$ with multiplicity one. The half plane $\Pi$ meets $\lim _{i \rightarrow \infty} \Gamma^{P, \lambda_{i}} \simeq \mathbb{R}^{2}$ orthogonally.

An analogous result holds for $P \in \mathcal{S} \cap \boldsymbol{U}$, in which case $\Pi$ is a plane.

Proof. By Proposition 4.3, Lemma 5.1] and Proposition 5.5, there exist a half plane $\Pi$ (with free boundary on $\lim _{i \rightarrow \infty} \Gamma^{P, \lambda_{i}} \simeq \mathbb{R}^{2}$ ) so that, after passing to a subsequence, $\left\{\boldsymbol{\Sigma}_{\tau}^{(P, T), \lambda_{i}}\right\} \rightarrow\{\Pi\}$ with multiplicity one away from $\mathfrak{S}_{P} \times(-\infty, 0)$, where $\mathfrak{S}_{P}$ is defined by (4.12).

Let $\widetilde{\boldsymbol{\Sigma}_{\tau}^{(P, T), \lambda_{i}}}$ be the reflection of $\boldsymbol{\Sigma}_{\tau}^{(P, T), \lambda_{i}}$ on $B_{\frac{1}{\lambda_{i^{\kappa}}}}(O)$ with respect to $\boldsymbol{\Gamma}^{P, \lambda_{i}}$, and define

$$
\overline{\boldsymbol{\Sigma}_{\tau}^{(P, T), \lambda_{i}}}=\boldsymbol{\Sigma}_{\tau}^{(P, T), \lambda_{i}} \cup \widetilde{\Sigma_{\tau}^{(P, T), \lambda_{i}}}
$$

By (4.10) and (4.11), $\overline{\boldsymbol{\Sigma}_{-1}^{(P, T), \lambda_{i}}}$ converges weakly to $\bar{\Pi}$ in the sense of varifolds as $i \rightarrow \infty$, where $\bar{\Pi}=\Pi \cup \tilde{\Pi}$ is a plane. Suppose that $\mathfrak{S}_{P} \neq \emptyset$, then pick $Q \in \mathfrak{S}_{P}$. By the weak convergence of varifolds and a similar argument as in Remark 4.2 , for almost every $r>0$, we have

$$
\frac{\mathcal{H}^{2}\left(\overline{\boldsymbol{\Sigma}_{-1}^{(P, T), \lambda_{i}}} \cap B_{r}(Q)\right)}{\pi r^{2}} \rightarrow 1 \quad \text { as } \quad i \rightarrow \infty .
$$

By (4.11) and Allard's regularity theorem (cf. [Al]), it follows that $\boldsymbol{\Sigma}_{-1}^{(P, T), \lambda_{i}}$ is a graph of a function whose $C^{1, \alpha}$ norm are uniformly bounded (independent of $i \in \mathbb{N}$ ) for any fixed $0<\alpha<1$. Applying Proposition 3.7 to the MCF $\left\{\boldsymbol{\Sigma}_{\tau}^{(P, T), \lambda_{i}}\right\}_{-1 \leq \tau<0}$, we then get a uniform bound (independent of $i \in \mathbb{N}$ ) on the second fundamental form of $\boldsymbol{\Sigma}_{-1}^{(P, T), \lambda_{i}}$ near $Q$. However, this contradicts with the choice of $Q$ (see (4.12) $)$. 


\section{Proof of the Main Theorem}

This section is devoted to prove our main theorem as stated below.

Theorem 6.1. The MCF $\left\{\boldsymbol{\Sigma}_{t}\right\}_{0 \leq t<T}$ given in Section 4 can be extended beyond time $T$.

Proof. By [S], it suffices to show that the second fundamental form of $\left\{\boldsymbol{\Sigma}_{t}\right\}_{0 \leq t<T}$ is uniformly bounded. Below we will prove that by contradiction.

Suppose that

$$
\limsup _{t \rightarrow T}\left\|A_{\boldsymbol{\Sigma}_{t}}\right\|_{L^{\infty}}=\infty
$$

Then choose a sequence $\left\{\left(P_{i}, t_{i}\right)\right\}_{i \in \mathbb{N}}$ so that $t_{i} \nearrow T, P_{i} \in \boldsymbol{\Sigma}_{t_{i}}$ and

$$
\left|A_{\boldsymbol{\Sigma}_{t_{i}}}\left(P_{i}\right)\right|=\sup _{0 \leq \tau \leq T-\frac{1}{i}}\left\|A_{\boldsymbol{\Sigma}_{\tau}}\right\|_{L^{\infty}} \rightarrow \infty \quad \text { as } i \rightarrow \infty .
$$

Since $\boldsymbol{\Sigma}_{0}$ is compact and $T<\infty$, (4.2) implies (after passing to a subsequence) that $P_{i} \rightarrow P$. Here we have three possibilities to consider:

- Case 1: $P \in \boldsymbol{U}$;

- Case 2: $P \in \boldsymbol{\Gamma}$ and $\liminf \inf _{i \rightarrow \infty} \operatorname{dist}\left(P_{i}, \partial \boldsymbol{\Sigma}_{t_{i}}\right)\left|A_{\boldsymbol{\Sigma}_{t_{i}}}\left(P_{i}\right)\right|<\infty$;

- Case 3: $P \in \boldsymbol{\Gamma}$ and $\liminf _{i \rightarrow \infty} \operatorname{dist}\left(P_{i}, \partial \boldsymbol{\Sigma}_{t_{i}}\right)\left|A_{\boldsymbol{\Sigma}_{t_{i}}}\left(P_{i}\right)\right|=\infty$.

Since Case 1 has been studied in $[\mathrm{LW}$ by using Proposition 5.5 and White's regularity theorem (cf. $[\mathrm{Wh}]$ ), we will focus on the remaining two cases. Actually, the argument for Case 2 is similar to that for Case 1.

Case $2\left(P \in \boldsymbol{\Gamma}\right.$ and $\left.\liminf \inf _{i \rightarrow \infty} \operatorname{dist}\left(P_{i}, \partial \boldsymbol{\Sigma}_{t_{i}}\right)\left|A_{\boldsymbol{\Sigma}_{t_{i}}}\left(P_{i}\right)\right|<\infty\right)$ :

By passing to a subsequence, we may assume that

$$
\operatorname{dist}\left(P_{i}, \partial \boldsymbol{\Sigma}_{t_{i}}\right) A_{i} \leq R<\infty
$$

for all $i \in \mathbb{N}$, where $A_{i}=\left|A_{\boldsymbol{\Sigma}_{t_{i}}}\left(P_{i}\right)\right|$. Choose $\stackrel{\circ}{P}_{i} \in \partial \boldsymbol{\Sigma}_{t_{i}}$ so that $\operatorname{dist}\left(P_{i}, \partial \boldsymbol{\Sigma}_{t_{i}}\right)=$ $\left|P_{i}-\stackrel{\circ}{P}_{i}\right|$. Clearly, we have $\stackrel{\circ}{P}_{i} \rightarrow P$ as $i \rightarrow \infty$.

Given $\varepsilon>0$, by Proposition 5.5, Lemma 2.6. Lemma 2.7 and the continuous dependence of the Gaussian integral on the parameters, there exists $\delta>0$ so that for $i \gg 1$ we have

$$
\frac{1}{2} \leq \sup _{t_{i}-\delta^{2} \leq t<t_{i}} \int_{\boldsymbol{\Sigma}_{t}} e^{85\left(\kappa^{2}\left(t_{i}-t\right)\right)^{\frac{2}{5}}} \eta_{\Gamma ; \stackrel{\circ}{i}_{i}, t_{i}} \Psi_{\Gamma ; \stackrel{P}{P}_{i}, t_{i}}(X, t) d \mathcal{H}^{2}(X) \leq \frac{1+\varepsilon}{2}
$$

(cf. [E]). After passing to a subsequence, we may assume that

$$
\frac{1}{2} \leq \sup _{t_{i}-\left(\frac{1}{A_{i}}\right)^{2} \leq t<t_{i}} \int_{\boldsymbol{\Sigma}_{t}} e^{85\left(\kappa^{2}\left(t_{i}-t\right)\right)^{\frac{2}{5}}} \eta_{\boldsymbol{\Gamma} ; \stackrel{P}{i}_{i}, t_{i}} \Psi_{\boldsymbol{\Gamma} ; \dot{P}_{i}, t_{i}}(X, t) d \mathcal{H}^{2}(X) \leq \frac{1+\frac{1}{i}}{2} \quad \forall i .
$$

Let

$$
\hat{\mathbf{\Sigma}}_{\tau}^{i}=A_{i}\left(\Sigma_{t_{i}+\frac{\tau}{A_{i}^{2}}}^{i}-\stackrel{\circ}{P}_{i}\right), \quad \mathcal{P}_{i}=A_{i}\left(P_{i}-\stackrel{\circ}{P}_{i}\right)
$$

Since $\mathcal{P}_{i} \in B_{2 R}(O)$ for $i \gg 1$, we may assume (by passing to a subsequence) that $\mathcal{P}_{i} \rightarrow \mathcal{P}$. In addition, we have

- $\sup _{-t_{i} A_{i}^{2} \leq \tau \leq 0}\left\|A_{\hat{\boldsymbol{\Sigma}}_{\tau}^{i}}\right\|_{L^{\infty}} \leq 1=\left|A_{\hat{\boldsymbol{\Sigma}}_{0}^{i}}\left(\mathcal{P}_{i}\right)\right|$;

- $\hat{\boldsymbol{U}}_{i}:=A_{i}\left(\boldsymbol{U}-\stackrel{\circ}{P}_{i}\right) \rightarrow \mathbb{R}_{+}^{3}, \quad \hat{\boldsymbol{\Gamma}}_{i}:=\partial \hat{\boldsymbol{U}}_{i}=A_{i}\left(\boldsymbol{\Gamma}-\stackrel{\circ}{P}_{i}\right)$ satisfies $\boldsymbol{\kappa}_{i}=\frac{\kappa}{A_{i}}$ graph condition and converges to $\partial \mathbb{R}_{+}^{3} \simeq \mathbb{R}^{2}$; 
- $\frac{1}{2} \leq \int_{\hat{\boldsymbol{\Sigma}}_{\tau}^{i}} e^{85\left(\boldsymbol{\kappa}_{i}^{2}(-\tau)\right)^{\frac{2}{5}}} \eta_{\hat{\boldsymbol{\Gamma}}_{i} ; O, 0} \Psi_{\hat{\boldsymbol{\Gamma}}_{i} ; O, 0}(X, \tau) d \mathcal{H}^{2}(X) \leq \frac{1+\frac{1}{i}}{2} \quad \forall-1 \leq \tau<0$. It follows from Proposition 2.15 that $\left\{\hat{\boldsymbol{\Sigma}}_{\tau}^{i}\right\}_{-t_{i} A_{i}^{2} \leq \tau \leq 0} \rightarrow\left\{\hat{\boldsymbol{\Sigma}}_{\tau}\right\}_{-\infty<\tau \leq 0}$. The limiting MCF has free boundary on $\lim _{i \rightarrow \infty} \hat{\boldsymbol{\Gamma}}_{i} \simeq \mathbb{R}^{2}$ and it satisfies

$$
\begin{aligned}
\left|A_{\hat{\boldsymbol{\Sigma}}_{0}}(\mathcal{P})\right| & =1, \\
\int_{\hat{\boldsymbol{\Sigma}}_{\tau}} \Psi_{\mathbb{R}^{2} ; O, 0}(X, t) d \mathcal{H}^{2}(X) & =\frac{1}{2} \quad \forall-1 \leq \tau<0 .
\end{aligned}
$$

Then Lemma 2.6 implies that $\left\{\hat{\boldsymbol{\Sigma}}_{\tau}\right\}_{-1 \leq \tau<0}$ is self-shrinking. Namely, it satisfies

$$
H_{\hat{\boldsymbol{\Sigma}}_{\tau}}+\frac{X_{\tau} \cdot N_{\hat{\boldsymbol{\Sigma}}_{\tau}}}{2(-\tau)}=0 \quad \forall X_{\tau} \in \hat{\boldsymbol{\Sigma}}_{\tau},-1 \leq \tau<0
$$

(cf. $[\mathrm{Wh}, \mathrm{B}]$ ). In particular, we get

$$
X_{\tau} \cdot N_{\hat{\boldsymbol{\Sigma}}_{\tau}}=2 \tau H_{\hat{\boldsymbol{\Sigma}}_{\tau}} \rightarrow 0 \quad \text { as } \tau \nearrow 0 .
$$

Consequently, $\hat{\boldsymbol{\Sigma}}_{0}$ must be a $C^{2}$ cone (with vertex at $O$ ) and hence a plane. This contradicts with the property that $\left|A_{\hat{\boldsymbol{\Sigma}}_{0}}(\mathcal{P})\right|=1$.

Case $3\left(P \in \boldsymbol{\Gamma}\right.$ and $\left.\liminf _{i \rightarrow \infty} \operatorname{dist}\left(P_{i}, \partial \boldsymbol{\Sigma}_{t_{i}}\right)\left|A_{\boldsymbol{\Sigma}_{t_{i}}}\left(P_{i}\right)\right|=\infty\right)$ :

Firstly, we claim that $\left|P-P_{i}\right| \geq \sqrt{T-t_{i}}$ for all but finitely many $i \in \mathbb{N}$. For otherwise, after passing to a subsequence, we would have

$$
\left|P-P_{i}\right|<\sqrt{T-t_{i}} \quad \forall i .
$$

By Corollary [5.6, $\left\{\frac{1}{\sqrt{T-t_{i}}}\left(\boldsymbol{\Sigma}_{t_{i}}-P\right)\right\}_{i \in \mathbb{N}}$ converges to a half-plane and hence

$$
\sqrt{T-t_{i}}\left|A_{\boldsymbol{\Sigma}_{t_{i}}}\left(P_{i}\right)\right| \rightarrow 0 \quad \text { as } \quad i \rightarrow \infty
$$

which implies

$\operatorname{dist}\left(\partial \boldsymbol{\Sigma}_{t_{i}}, P\right) \geq \operatorname{dist}\left(\partial \boldsymbol{\Sigma}_{t_{i}}, P_{i}\right)-\left|P-P_{i}\right| \gg\left|A_{\boldsymbol{\Sigma}_{t_{i}}}\left(P_{i}\right)\right|^{-1}-\sqrt{T-t_{i}} \gg \sqrt{T-t_{i}}$

for $i \gg 1$. However, (4.2) yields that

$$
\operatorname{dist}\left(\partial \boldsymbol{\Sigma}_{t_{i}}, P\right) \leq \boldsymbol{\Lambda}\left(T-t_{i}\right)
$$

(cf. $[\mathrm{K}]$ ). Thus, we get a contradiction.

By passing to a subsequence, let's assume that

$$
\left|P-P_{i}\right| \geq \sqrt{T-t_{i}} \quad \forall i .
$$

It follows from (6.1) and (6.2) that

$$
\operatorname{dist}\left(P, \partial \boldsymbol{\Sigma}_{t_{i}}\right) \leq \boldsymbol{\Lambda}\left(T-t_{i}\right) \ll \sqrt{T-t_{i}} \leq\left|P-P_{i}\right|
$$

for $i \gg 1$, which implies

$$
\left|P_{i}-P\right| \geq \operatorname{dist}\left(P_{i}, \partial \boldsymbol{\Sigma}_{t_{i}}\right)-\operatorname{dist}\left(P, \partial \boldsymbol{\Sigma}_{t_{i}}\right) \geq \operatorname{dist}\left(P_{i}, \partial \boldsymbol{\Sigma}_{t_{i}}\right)-\frac{1}{2}\left|P_{i}-P\right| .
$$

Thus we have

$$
\left|P_{i}-P\right| \geq \frac{2}{3} \operatorname{dist}\left(P_{i}, \partial \boldsymbol{\Sigma}_{t_{i}}\right) .
$$


Let $C \geq 1$ be a large number to be determined. For $i \gg 1$, let $\lambda_{i}=C\left|P-P_{i}\right|$, then Corollary 5.6 implies that

$$
\boldsymbol{\Sigma}_{\tau}^{(P, T), \lambda_{i}}:=\frac{1}{\lambda_{i}}\left(\boldsymbol{\Sigma}_{T+\lambda_{i}^{2} \tau}-P\right)
$$

converges to a half-plane $\Pi$ with multiplicity one as $i \rightarrow \infty$. In particular, we have

$$
\left\|A_{\Sigma_{-1}^{(P, T), \lambda_{i}}}\right\|_{L^{\infty}\left(B_{1}(O)\right)} \leq 1
$$

for $i \gg 1$. Moreover, since

$$
\sup _{-\frac{T}{\lambda_{i}^{2}} \leq \tau<0}\left\|H_{\boldsymbol{\Sigma}_{\tau}^{(P, T), \lambda_{i}}}\right\|_{L^{\infty}} \leq \lambda_{i} \boldsymbol{\Lambda}
$$

and $\boldsymbol{\Gamma}^{P, \lambda_{i}}$ satisfies a $\lambda_{i} \boldsymbol{\kappa}$-graph condition, Proposition 3.5 implies that there is $0<\delta<1$ so that

$$
\begin{gathered}
\sup _{0<r \leq \delta} \frac{\mathcal{H}^{2}\left(\boldsymbol{\Sigma}_{\tau}^{(P, T), \lambda_{i}} \cap B_{r}(Q)\right)_{Q}+\mathcal{H}^{2}\left(\left(\boldsymbol{\Sigma}_{\tau}^{(P, T), \lambda_{i}} \cap B_{r}(Q)\right)_{Q} \cap \tilde{B}_{r}(Q)\right)}{\pi r^{2}} \leq 1+\vartheta \\
\forall Q \in \boldsymbol{\Sigma}_{\tau}^{(P, T), \lambda_{i}} \cap B_{\delta}(O), \quad-1 \leq \tau<\min \left\{0,-1+\frac{\delta}{\lambda_{i} \Lambda}\right\}=0, \quad i \gg 1,
\end{gathered}
$$

where $\vartheta>0$ is the constant in Lemma 3.6. Note that the reflection $\tilde{B}_{r}(Q)$ is with respect to $\boldsymbol{\Gamma}^{P, \lambda_{i}}:=\frac{1}{\lambda_{i}}(\boldsymbol{\Gamma}-P)$ (see Definition 2.1).

Let $A_{i}=\left|A_{\boldsymbol{\Sigma}_{t_{i}}}\left(P_{i}\right)\right|$ and define

$$
\hat{\mathbf{\Sigma}}_{\tau}^{i}=A_{i}\left(\boldsymbol{\Sigma}_{t_{i}+\frac{\tau}{A_{i}^{2}}}-P_{i}\right)
$$

then we have

$$
\begin{gathered}
\sup _{t_{i} A_{i}^{2} \leq \tau \leq 0}\left\|A_{\hat{\boldsymbol{\Sigma}}_{\tau}^{i}}\right\|_{L^{\infty}} \leq 1=\left|A_{\hat{\boldsymbol{\Sigma}}_{0}^{i}}(O)\right|, \\
\sup _{t_{i} A_{i}^{2} \leq \tau \leq 0}\left\|H_{\hat{\boldsymbol{\Sigma}}_{\tau}^{i}}\right\|_{L^{\infty}} \leq \frac{\boldsymbol{\Lambda}}{A_{i}} .
\end{gathered}
$$

Note that

$$
\hat{\mathbf{\Sigma}}_{0}^{i}=\lambda_{i} A_{i} \boldsymbol{\Sigma}_{\tau_{i}}^{(P, T), \lambda_{i}}+A_{i}\left(P-P_{i}\right),
$$

where

$$
\begin{gathered}
\lambda_{i} A_{i}=C\left|P-P_{i}\right|\left|A_{\boldsymbol{\Sigma}_{t_{i}}}\left(P_{i}\right)\right| \geq \frac{2}{3} C \operatorname{dist}\left(\partial \boldsymbol{\Sigma}_{t_{i}}, P_{i}\right)\left|A_{\boldsymbol{\Sigma}_{t_{i}}}\left(P_{i}\right)\right| \rightarrow \infty, \\
A_{i}\left|P-P_{i}\right| \geq \frac{2}{3} \operatorname{dist}\left(P_{i}, \partial \boldsymbol{\Sigma}_{t_{i}}\right)\left|A_{\boldsymbol{\Sigma}_{t_{i}}}\left(P_{i}\right)\right| \rightarrow \infty
\end{gathered}
$$

by (6.3), and

$$
\tau_{i}=-\frac{T-t_{i}}{\lambda_{i}^{2}} \in\left[-C^{-2}, 0\right)
$$

by (6.2). Furthermore, given $\mathcal{Q} \in \hat{\boldsymbol{\Sigma}}_{0}^{i} \cap B_{\frac{1}{2} A_{i}\left|P-P_{i}\right|}(O)$ and $R>0$, (6.4) and (6.5) yield

$$
\frac{\mathcal{H}^{2}\left(\hat{\boldsymbol{\Sigma}}_{0}^{i} \cap B_{R}(\mathcal{Q})\right)_{\mathcal{Q}}+\mathcal{H}^{2}\left(\left(\hat{\boldsymbol{\Sigma}}_{0}^{i} \cap B_{R}(\mathcal{Q})\right)_{\mathcal{Q}} \cap \tilde{B}_{R}(\mathcal{Q})\right)}{\pi R^{2}}
$$


$=\frac{\mathcal{H}^{2}\left(\boldsymbol{\Sigma}_{\tau_{i}}^{(P, T), \lambda_{i}} \cap B_{\frac{R}{\lambda_{i} A_{i}}}(Q)\right)_{Q}+\mathcal{H}^{2}\left(\left(\boldsymbol{\Sigma}_{\tau_{i}}^{(P, T), \lambda_{i}} \cap B_{\frac{R}{\lambda_{i} A_{i}}}(Q)\right)_{Q} \cap \tilde{B}_{\frac{R}{\lambda_{i} A_{i}}}(Q)\right)}{\pi\left(\frac{R}{\lambda_{i} A_{i}}\right)^{2}} \leq 1+\vartheta$

for $i \gg 1$, where

$$
Q=\frac{1}{\lambda_{i}}\left(P_{i}-P+\frac{\mathcal{Q}}{A_{i}}\right) \in \boldsymbol{\Sigma}_{\tau_{i}}^{(P, T), \lambda_{i}} \cap\left(B_{\frac{3}{2} C^{-1}}(O) \backslash B_{\frac{1}{2} C^{-1}}(O)\right) .
$$

Note that $\left(B_{\frac{3}{2} C^{-1}}(O) \backslash B_{\frac{1}{2} C^{-1}}(O)\right)=B_{\delta}(O) \backslash B_{\frac{\delta}{3}}(O)$ if we choose $C=\frac{3}{2} \delta^{-1}$, and that the reflection in the first ratio (i.e. $\left.\tilde{B}_{R}(\mathcal{Q})\right)$ is with respect to $\hat{\boldsymbol{\Gamma}}^{i}=A_{i}\left(\boldsymbol{\Gamma}-P_{i}\right)$. Passing to a subsequence, we have $\left\{\hat{\boldsymbol{\Sigma}}_{\tau}^{i}\right\} \rightarrow\{\hat{\boldsymbol{\Sigma}}\}_{-\infty<\tau \leq 0}$, where $\hat{\boldsymbol{\Sigma}}$ is a complete minimal surface (with free boundary on $\lim _{i \rightarrow \infty} \hat{\boldsymbol{\Gamma}}^{i}$ if $\lim _{i \rightarrow \infty} \hat{\boldsymbol{\Gamma}}^{i} \neq \emptyset$ ). The limiting minimal surface satisfies

$$
\begin{gathered}
\sup _{R>0} \sup _{\mathcal{Q} \in \hat{\boldsymbol{\Sigma}}} \frac{\mathcal{H}^{2}\left(\hat{\boldsymbol{\Sigma}} \cap B_{R}(\mathcal{Q})\right)_{\mathcal{Q}}+\mathcal{H}^{2}\left(\left(\hat{\boldsymbol{\Sigma}} \cap B_{R}(\mathcal{Q})\right)_{\mathcal{Q}}+\tilde{B}_{R}(\mathcal{Q})\right)}{\pi R^{2}} \leq 1+\vartheta, \\
\left\|A_{\hat{\boldsymbol{\Sigma}}}\right\|_{L^{\infty}} \leq 1=\left|A_{\hat{\boldsymbol{\Sigma}}}(O)\right| .
\end{gathered}
$$

A contradiction follows from Lemma 3.6 .

\section{REFERENCES}

[Al] W. Allard: On the first variation of a varifold, Ann. Math., 95 (1972), 417-491.

[An] B. Andrews: Fully nonlinear parabolic equations in two space variables, arXiv:math/0402235 1 .

[AS] D. Aronson, J. Serrin: Local behavior of solutions of quasilinear parabolic equations, Arch. Rational Mech. Anal. 25 1967, 81-122.

[B] J. Buckland: Mean curvature flow with free boundary on smooth hypersurfaces, J. Reine Angew. Math. 586 (2005), 71-90.

[C] A. Cooper: A characterization of the singular time of the mean curvature flow, Proc. Amer. Math. Soc. 139 (2011), no. 8, 29332942.

[CM] T. Colding, W. Minicozzi II: Smooth compactness of self-shrinkers, Comment. Math. Helv. 87 (2012), no. 2, 463-475.

[E] K. Ecker: Regularity theory for mean curvature flow, Birkhäuser, Boston (2004).

[EH] K. Ecker, G. Huisken: Interior estimates for hypersurfaces moving by mean curvature, Invent. math. 105, 547-569 (1991).

[GJ] M. Grüter, J. Jost: Allard type regularity results for varifolds with free boundaries, Ann. Scu. Norm. Sup. Pisa Cal. Sci (4) 13 (1986), n. 1, 129-169.

[H1] G. Huisken: Non-parametric mean curvature evolution with boundary conditions, J. Differ. Eqns.77 (1989) 369-378.

[H2] G. Huisken: Asymptotic behaviour for singularities of the mean curvature flow, J. Diff. Geom. 31, 285-299 (1990).

[HS] G. Huisken, C. Sinestrari: Convexity estimates for mean curvature flow and singularities of mean convex surfaces, Acta Math. 183 (1999), no. 1, 45-70.

[I] T. Ilmanen: Singularities of mean curvature flow of surfaces, preprint.

[K] A. Koeller: Regularity of mean curvature flows with Neumann free boundary conditions, Calc. Var. (2012) 43:265-309.

[LS] N. Le, N. Sesum: The mean curvature at the first singular time of the mean curvature flow, Ann. Inst. H. Poincaré Anal. Non Linéaire 27 (2010), no. 6, 14411459.

[LW] H. Li, B. Wang: The extension problem of the mean curvature flow (I), arXiv:1608.02832.

[LZZ] Y. Leng, E. Zhao, H. Zhao: Notes on the extension of the mean curvature flow, Pacific Journal of Mathematics 269 (2), 385-392, 2014. 1, 2014. 
[PR] J. Pérez, A. Ros: Properly embedded minimal surfaces with finite total curvature. The global theory of minimal surfaces in flat spaces (Martina Franca, 1999), 15-66, Lecture Notes in Math., 1775, Fond. CIME/CIME Found. Subser., Springer, Berlin, 2002.

[S] A. Stahl: Regularity estimates for solutions to the mean curvature flow with a Neumann boundary condition, Calc. Var. 4, 385-407 (1996).

[Wa] W. Walter: On the strong maximum principle for parabolic differential equations, Proc. Edinburgh Math. Soc. (2) 29 (1986), no. 1, 93-96.

[Wh] B. White: A local regularity theorem for mean curvature flow, Ann. of Math. (2) 161 (2005), no. 3, 1487-1519.

Department of Mathematics, Indiana University - Rawles Hall

831 East 3rd St. Bloomington, IN 47405-7106

E-mail address: siaoguo@iu.edu 